\title{
The Effect of Viscoelasticity on the Life Expectancy of Light Composite Aircraft
}

\author{
by \\ Christopher Goudie, B. Eng.

\begin{abstract}
A thesis submitted to the
Faculty of Graduate and Postdoctoral Affairs

in partial fulfillment of the requirements for the degree of
\end{abstract}

Master of Applied Science in Aerospace Engineering

Ottawa-Carleton Institute for Mechanical and Aerospace Engineering

Department of Mechanical and Aerospace Engineering

Carleton University

Ottawa, Ontario

January, 2015

(C) Copyright

Christopher Goudie, 2015 


\section{Abstract}

In modern aircraft, polymer matrix composites (PMC) are becoming more prevalent in both primary and secondary structures due to their high strength and stiffness to weight ratios. Due to the molecular composition of polymers, these materials exhibit viscoelastic phenomena such as creep, recovery and relaxation. The scope of this thesis is to predict the service life of the Diamond DA-20 Katana based on the viscoelastic properties of the MSG L285/H287 epoxy matrix. The prediction methodology is applied to the composite wing spar because the spar bears the majority of the load during flight. The methodology requires the experimental characterization of the epoxy matrix, which was achieved by retrofitting a decommissioned undergraduate laboratory furnace. The retrofit created a novel creep and relaxation measurement apparatus. Using elevated temperature, the long term response of the epoxy matrix was determined using the time-temperature superposition principle. The results were then applied to the spar to determine its long term response using the Classical Lamination Theory applied to complex geometries. Failure was determined based on the Tsai-Hill and maximum strain failure theory or if the compressive flange buckles under the applied load.

Based on the analysis, it was determined that the MSG L285/H287 epoxy system adheres to the Time-Temperature Superposition principal and master curves were generated using a limited data set. Composite analysis was performed on the wing spar using the viscoelastic properties of the epoxy. Based on the analysis presented in this thesis, it is concluded that Diamond's claim's of unlimited service life is possible based on the assumptions made with respect to viscoelastic phenomena. 


\section{Acknowledgments}

I would like to acknowledge and thank the following individuals for their support in my research.

- Professor J. Laliberte for his insight in the analysis of composite materials and the composite industry.

- Steve Truttmann for his help with material testing procedures and training on the MTS equipment.

- Finally, my parents Gord and Quyen Goudie who have fully supported me throughout my studies. 


\section{Table of Contents}

Abstract $\quad$ ii

Acknowledgments $\quad$ iii

Table of Contents $\quad$ iv

List of Tables $\quad$ vii

List of Figures viii

Nomenclature $\quad$ xi

1 Introduction $\quad 1$

1.1 Motivation ........................ 1

1.2 Description of the Aircraft . . . . . . . . . . . . . . . 2

2 Review of Composite Material use the Aviation Industry 7

2.1 Manufacturing of Composite Materials . . . . . . . . . . . . . . . . . 9

2.2 Service Life Prediction . . . . . . . . . . . . . . . . . . . . . . 10

2.2.1 Damage and its Effect on Services Life . . . . . . . . . . . . . 12

2.2.2 Fatigue and its Effect on Service Life . . . . . . . . . . . . 13

2.2.3 Viscoelasticity and its Effect on Services Life . . . . . . . . . . 14

2.3 Testing Methods for Viscoelastic Materials . . . . . . . . . . . . 15

3 Theory of Viscoelastic and Composite Materials 17

3.1 Characteristics of Polymers . . . . . . . . . . . . . . . . . 17

3.2 Properties of Viscoelastic Materials . . . . . . . . . . . . . . . 19

3.2.1 Experimentally Determined Creep and Relaxation Characteristics 22

3.2.2 Effect of Temperature . . . . . . . . . . . . 23 
3.2.3 Generation of Master Curves . . . . . . . . . . . . . . 26

3.2.4 Time Dependent Nature of Poisson's Ratio . . . . . . . . . . 28

3.3 Characteristics of Composite Materials _ . . . . . . . . . . . 30

3.3 .1 Classical Lamination Theory . . . . . . . . . . . . . . . . . 30

3.3.2 Properties with Respect to Global Frame of Reference . . . . . 32

3.3.3 Engineering Constants of a Laminated Plate . . . . . . . . . 35

3.3.4 Determination of the Effective Axial Stiffness . . . . . . . 37

3.3.5 Determination of the Effective Flexural Stiffness . . . . . . . . 40

3.3.6 Determination of the Effective Torsional Rigidity . . . . . . . 43

3.4 Wing Deflection Given Arbitrary Loading Profile . . . . . . . . . 46

3.5 Failure Criterion . . . . . . . . . . . . . . . . . . . 47

3.5 .1 Tsai-Hill Failure Theory _ . . . . . . . . . . . . . . 47

3.5.2 Maximum Strain Theory . . . . . . . . . . . . . . . . . . 48

3.5.3 Local buckling of the Flange or Web . . . . . . . . . . 48

4 Experimental Setup $\quad 52$

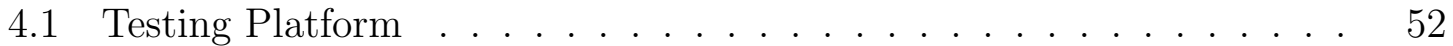

4.2 National Instruments LabVIEW . . . . . . . . . . . . . . . . 54

4.3 Sensors and Actuation Control . . . . . . . . . . . . . . . 56

4.4 Specimen Manufacturing and Testing Procedure . . . . . . . . . . 59

5 Results and Analysis $\quad 64$

5.1 Viscoelastic Response of the Epoxy Resin . . . . . . . . . . . . 64

5.2 Response of Composite Wing Spar . . . . . . . . . . . . . . 73

5.2 .1 Determination of Ply Layup . . . . . . . . . . . . . . . 74

5.3 Elastic Analysis of Composite Wing Spar . . . . . . . . . . . 75

5.4 Viscoelastic Analysis the Wing Spar . . . . . . . . . . . . . . 79

5.4.1 Viscoelastic Response During Steady Level Flight . . . . . . . 79

5.4 .2 Viscoelastic Failure Analysis . . . . . . . . . . . . . . 80

5.5 Summary of Service Life Prediction Using Viscoelastic Properties . . 82

6 Potential Improvements and Future Work 84

6.1 Limitations of the Current Test Setup . . . . . . . . . . . . . . . 84

6.2 General Parameters for Custom Oven . . . . . . . . . . . . . . . . 86

6.3 Future Work . . . . . . . . . . . . . . . . . . . . . 88 
$\begin{array}{llr}7 & \text { Conclusion } & 90\end{array}$

7.1 Viscoelastic Response of MGS Epoxy System . . . . . . . . . . . . . . 90

7.2 Response of Composite Wing Spar . . . . . . . . . . . . . . . . . . 91

7.3 Improvements, Future Work and Concluding Remarks . . . . . . . . . 92

$\begin{array}{ll}\text { List of References } & 93\end{array}$ 


\section{List of Tables}

2.1 Common manufacturing techniques used to fabricate composite com-

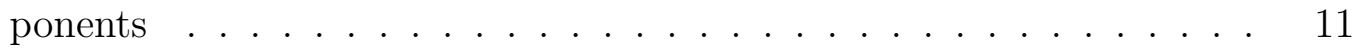

4.1 Calibration coefficients to convert sensor voltage to their respective units 58

5.1 Comparison of temperature to respective time shift for epoxy resin given a $25^{\circ} \mathrm{C}$ reference temperature and $270 \frac{\mathrm{kJ}}{\mathrm{mol} \cdot \mathrm{K}}$ activation energy . 66

5.2 Important Dimensions of the Diamond DA-20 Katana . . . . . . . . . 75

5.3 Elastic properties of the raw materials used to fabricate the composite wing spar . . . . . . . . . . . . . . . . . 76

5.4 Table listing strength ratio of the weakest ply within each section of the I-beam using elastic properties and the Tsai-Hill and Maximum strain failure theory . . . . . . . . . . . . . . . 78

5.5 Comparisons of the wing spars response using elastic and viscoelastic properties . . . . . . . . . . . . . . . . . . . . 79

5.6 Table listing strength ratio of the weakest ply within each section of the I-beam using the relaxed properties, Tsai-Hill failure theory and a varying ratio of epoxy strength . . . . . . . . . . . . .

5.7 Table listing strength ratio of the weakest ply within each section of the I-beam when compared to the maximum allowable elongation of the fibres using relaxed properties . . . . . . . . . . . . . . . . . . . 82

5.8 Comparisons of the critical buckling moment for local buckling of the compressive flange to occur and their respective safety factor . . . . . 


\section{List of Figures}

1.1 Research components of the Diamond DA-20 Katana life prediction project . . . . . . . . . . . . . . . . 2

1.2 Photograph of Diamond DA-20 Katana . . . . . . . . . . . . . . . 3

1.3 Schematic diagram of Diamond DA20-A1 Katana . . . . . . . . . . 4

1.4 Schematic diagram and end view of the Diamond DA20-A1 wing spar 5

1.5 Flow chart depicting the interrelationship between each topic . . . . . 6

2.1 Illustration of the increased percentage of composite use in structural components for aircraft since 1960 . . . . . . . . . . . . 8

2.2 Interrelation between manufacturing, performance and cost in the design process . . . . . . . . . . . . . . . . . 10

2.3 Illustration of common forms damage - A) Delamination, B) Fibre/matrix debonding, C) Matrix microcracking . . . . . . . . . . . . 12

2.4 Various degrees of Impact Damage encountered by composite structures 13

2.5 Comparison showing the complex nature of fatigue damage in composites compare to that found in metals during a notch test . . . . . . . 14

2.6 Various viscoelastic testing apparatus: (A) - Instatron Stress Rupture Testing System - Model C, (B) - TA Instruments RSA-G2 Solid Analyser, (C) - Rheolution Instruments Hyper Frequency Viscoelastic Spectroscopy . . . . . . . . . . . . . . . . 16

3.1 Simplified classification scheme of engineering polymers . . . . . . . 17

3.2 Generalized micro-structure comparing thermoplastic and thermoseting polymers . . . . . . . . . . . . . . . . . 18

3.3 Various examples of viscoelastic materials common to our society . . 19

3.4 Creep response of thermosetting and thermoplastic polymers given constant stress . . . . . . . . . . . . . . . . . . . . 20

3.5 Creep and recovery response of thermosetting and thermoplastic polymers given constant stress . . . . . . . . . . . . . . . . . 20 
3.6 Relaxation curves for thermosetting and thermoplastic polymers given a constant strain . . . . . . . . . . . . . . . . . . . . . 21

3.7 Illustration of the Maxwell and Kelvin viscoelastic models . . . . . . 22

3.8 Measured relaxation function for polyisobutylene (PIB) and the series expansion fit . . . . . . . . . . . . . . . . . . . 23

3.9 Relaxation modulus with respect to temperature for various types of polymers: A) Crystalline polystyrene, B) Cross-linked polystyrene, C) Amorphous polystyrene. . . . . . . . . . . . . . . . . 24

3.10 A general example of the effect of decreasing the relaxation time by increasing the test temperature . . . . . . . . . . . . 25

3.11 Illustration of thermal oxidation to a test specimen when exposed to high temperature for an extended period of time: Top - New specimen, Bottom - Thermally oxidized specimen . . . . . . . . . . . . 27

3.12 Hypothetical construction of a viscoelastic master curve for PIB at $25^{\circ} \mathrm{C}$ reference temperature . . . . . . . . . . . . . . 27

3.13 Comparison of the compliance and the Poisson's ratio for a viscoelastic material against the $\log$ of time . . . . . . . . . . . . . . . . . 28

3.14 Illustration of the local frame of reference for a single ply . . . . . . . 30

3.15 Illustration of the representative volume element assumption . . . . . 31

3.16 Notation used to describe stacking sequence of plies . . . . . . . . . . 33

3.17 Graphical illustration of notation used to describe to the I-beam . . . 38

3.18 Free body diagram illustrating the loads imposed by each component of the I beam in pure extension . . . . . . . . . . . . . . . . . . . . 38

3.19 Free body diagram illustrating the loads imposed by each component of the I-beam in pure bending . . . . . . . . . . . . . . . . . . . . . . 40

3.20 Free body diagram illustrating the shear loads imposed on components of the I-beam in pure torsion . . . . . . . . . . . . . . . . . . . . . . 44

3.21 Initial local buckling of the compressive flange . . . . . . . . . . . . 48

3.22 Ultimate failure of the web and beam following the buckling of the flange 49

3.23 Illustration of the boundary conditions imposed on the web (A) and the half flange $(\mathrm{B})$. . . . . . . . . . . . . . . . 49

4.1 Existing undergraduate equipment used to demonstrate creep with lead. 53

4.2 Former set up for the creep and relaxation tests . . . . . . . . . . . . 54

4.3 National Instrument USB-6008 Data acquisition unit . . . . . . . . . 55 
4.4 Example of LabVIEW programing interface . . . . . . . . . . . . . . 55

4.5 Approximate loading cycle for the creep and relaxation tests . . . . . 56

4.6 Simplified wiring diagram to connects the various sensor to the USB6008 and their respective power source . . . . . . . . . . . . . 57

4.7 Circuit diagram of basic H-Bridge motor controller . . . . . . . . . . 59

4.8 Simplified wiring diagram to control the 2500N linear actuator . . . . 60

4.9 ASTM International recommendation for plastic test specimens . . . 61

4.10 Desired dimensions for custom specimens using neat resin . . . . . . . 61

4.11 Completed epoxy specimen used in the creep and relaxation tests . . 62

5.1 Loading data used to measure the initial modulus of the specimen . . 65

5.2 Master creep compliance curve generated for a reference temperature of $25^{\circ} \mathrm{C} \ldots \ldots \ldots \ldots$

5.3 Master relaxation stiffness curve generated for a reference temperature

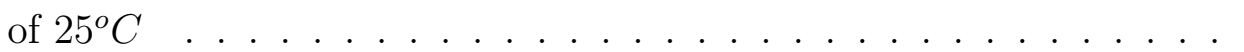

5.4 Master creep compliance curve generated using various activation energies . . . . . . . . . . . . . . . . . . . . 71

5.5 Comparison of master curves generated for $25^{\circ} \mathrm{C}$ from creep and relaxation data normalized for stiffness . . . . . . . . . . . . . . . . 72

5.6 Creep compliance master curve using instantaneous temperature to determine shift factor . . . . . . . . . . . . . . . . . 73

5.7 Assumed stacking sequence for the top and bottom flanges . . . . . . 74

5.8 Assumed stacking sequence of the web . . . . . . . . . . . . 75

5.9 Graphical representation of the wing's vertical deflection during steady level flight, including a reference to the aircraft cockpit . . . . . . . . 76

5.10 Quadrant breakdown of I beam for stress analysis . . . . . . . . . . . 77

6.1 Comparison of past test equipment for lead, on the left, with the new equipment for polymers on the right . . . . . . . . . . . . . 85

6.2 Conceptual drawing of the redesigned test setup . . . . . . . . . . . 88 


\section{Nomenclature}

$\begin{array}{ll}\alpha & \text { Relaxation time constant, }{ }^{1} / s \\ \gamma & \text { Shear strain } \\ \gamma^{o} & \text { Shear strain at mid-plane of composite lamina } \\ \varepsilon & \text { Strain } \\ \varepsilon_{x, y, x y} & \text { Strain global frame of reference } \\ \varepsilon_{1,2,12} & \text { Strain local frame of reference } \\ \varepsilon^{o} & \text { Strain at mid-plane of composite lamina } \\ \eta & \text { Viscoelastic damping coefficient } \\ \kappa & \text { Mid-plane curvature } \\ \sigma & \text { Stress, MPa } \\ \sigma_{x, y, z} & \text { Stress in global frame of reference, MPa } \\ \sigma_{1,2,3} & \text { Stress in local frame of reference, MPa } \\ \left.\sigma_{f, C}^{T, C}\right|_{u l t} & \text { Ultimate tensile and compressive strength of the fibre and matrix, MPa } \\ \left.\sigma_{1,2}^{T, C}\right|_{u l t} & \text { Ultimate tensile and compressive strength in the local frame of reference, MPa } \\ \nu & \text { Poisson's Ratio } \\ \nu_{f, m} & \text { Poisson's Ratio of fibre and matrix materials } \\ \nu_{x y} & \text { Poisson's Ratio global frame of reference } \\ \nu_{12} & \text { Poisson's Ratio local frame of reference } \\ \psi & \text { Angle of twist, deg } \\ \tau & \text { Shear stress, MPa } \\ \tau_{x y} & \text { Shear stress in the global frame of reference, MPa } \\ \tau_{12} & \text { Shear stress in the local frame of reference, MPa } \\ \left.\tau_{f, m}\right|_{\text {ult }} & \text { Shear strength of fibre and matrix materials, MPa } \\ \left.\tau_{12}\right|_{u l t} & \text { Shear strength in local frame of reference, MPa } \\ \theta & \text { Ply angle, degree } \\ \xi & \text { Dummy variable of integration } \\ \xi_{f l a n g e} & \text { Coefficient of Restraint } \\ & \end{array}$




\begin{tabular}{|c|c|}
\hline$C_{1}, C_{2}$ & William-Landel-Ferry coefficients \\
\hline$A_{w, t f, b f}$ & Cross sectional area of the web, top and bottom flanges, $m^{2}$ \\
\hline$E$ & Young's Modulus, GPa \\
\hline$E_{m, f}$ & Young's Modulus of fibre and matrix, GPa \\
\hline$E_{x, y, x y}$ & Young's Modulus global frame of reference, GPa \\
\hline$E_{1,2,12}$ & Young's Modulus local frame of reference, GPa \\
\hline$E_{a}$ & Activation Energy, kJ \\
\hline$\left.E_{x}^{e x t}\right|_{w, t f, b f}$ & Extensional modulus of the web, top and bottom flanges, GPa \\
\hline$\left.E_{x}^{f l e x}\right|_{w, t f, b f}$ & Flexural modulus of the web, top and bottom flanges, GPa \\
\hline$\overline{E I}$ & Effective flexural rigidity of the composite beam, $N \cdot m^{2}$ \\
\hline$G$ & Shear Modulus, GPa \\
\hline$G_{m, f}$ & Shear Modulus of fibre and matrix, GPa \\
\hline$G_{x, y, x y}$ & Shear Modulus global frame of reference, GPa \\
\hline$G_{1,2,12}$ & Shear Modulus local frame of reference, GPa \\
\hline$\overline{G J}$ & Effective torsional rigidity of the composite beam, $N \cdot m^{2}$ \\
\hline$I$ & Second moment of area, $\mathrm{mm}^{4}$ \\
\hline$L$ & Length of beam, $m$ \\
\hline$M(x)$ & Moment distribution, $N \cdot m$ \\
\hline$M$ & Equivalent moment, $N$ \\
\hline$\left.M_{x}\right|_{w, t f, b f}$ & Equivalent moment induced by web, top and bottom flanges, $\mathrm{N}$ \\
\hline$\left.M_{x}^{t o t a l}\right|_{w, t f, b f}$ & Total moment acting on composite lamina, $\mathrm{N} \cdot \mathrm{m}$ \\
\hline$N$ & Equivalent axial force, ${ }^{N} / \mathrm{mm}$ \\
\hline$\left.N_{x}\right|_{w, t f, b f}$ & Equivalent axial force acting on web, top and bottom flanges \\
\hline$R$ & Universal Gas Constant, ${ }^{k} \mathrm{~J} / \mathrm{mol} \cdot \mathrm{K}$ \\
\hline$T$ & Temperature, ${ }^{\circ} \mathrm{C}$ \\
\hline$T_{g}$ & Glass Transition Temperature, ${ }^{\circ} \mathrm{C}$ \\
\hline$T_{\text {ref }}$ & Reference temperature, ${ }^{\circ} \mathrm{C}$ \\
\hline$T_{c}$ & Total torque acting through the centroid, $N \cdot m$ \\
\hline$\left.T^{t o t a l}\right|_{w, t f, b f}$ & Total torque supported by the web, top and bottom flanges $N \cdot m$ \\
\hline$V_{f, m}$ & Volume fraction of fibre and matrix \\
\hline$J(t)$ & Relaxation function, $G P a$ \\
\hline$Y(t)$ & Compliance function, ${ }^{1} / G P a$ \\
\hline$[A]$ & Extensional stiffness matrix, GPa \\
\hline$[B]$ & Coupling stiffness matrix, GPa \\
\hline
\end{tabular}




$\begin{array}{ll}{[D]} & \text { Flexural stiffness matrix, GPa } \\ {[a]} & \text { Inverse of extensional stiffness matrix, } 1 / G P a \\ {[d]} & \text { Inverse of flexural stiffness matrix, }{ }^{1} / G P a \\ {[S]} & \text { Compliance matrix of lamina in local frame of reference, GPa } \\ {[\bar{S}]_{\theta}} & \text { Compliance matrix of laminated plate in global frame of reference, GPa } \\ {[Q]} & \text { Stiffness matrix of lamina in local frame of reference, GPa } \\ {[\bar{Q}]_{\theta}} & \text { Stiffness matrix of laminated plate in global frame of reference, GPa } \\ {[T]_{\theta}} & \text { Transformation matrix for stress calculations for ply angle } \theta \\ {\left[T^{*}\right]_{\theta}} & \text { Transformation matrix for strain calculations for ply angle } \theta \\ a_{t} & \text { Time-temperature shift factor } \\ b_{w, t f, b f} & \text { Length of web and top, bottom flange sections, } m m \\ h & \text { Thickness, mm } \\ k_{f l a n g e} & \text { Effective spring constant of compressive flange } \\ m & \text { Mass, kg } \\ t & \text { Thickness, mm } \\ t & \text { Time, s } \\ z_{c} & \text { Location of centroid, mm } \\ \bar{z} & \text { Distance from mid-plane, mm }\end{array}$

\section{Common Subscript}

$f \quad$ Fibre properties

$g \quad$ Glass temperature

$i \quad$ Variable number

$m \quad$ Matrix properties

o Initial conditions

$k \quad$ Composite ply number

$w, t f, b f \quad$ Components of I-beam - web, top and bottom flange

$x, y, z \quad$ Global frame of reference

1,2,3 Local frame of reference 


\section{Chapter 1}

\section{Introduction}

In modern aircraft, polymer matrix composites (PMC) are becoming more prevalent in both primary and secondary structures due to their high strength and stiffness to weight ratios [1]. Typically these are comprised of a polymer matrix with a reinforcement. The molecular make-up of polymers leads to viscoelastic properties even at room temperatures thus the stresses and deformations of the material are dependent upon load, time, temperature, and load history. [1]. Consequently, an accurate life span for aircraft manufactured from polymer composites can be difficult to predict using elastic models. One such aircraft is the Diamond DA20-A1 Katana which is manufactured entirely from glass and carbon fibre reinforced plastic material [2].

The focus of this thesis is to predict the service life of this aircraft, defined as the time required for the composite wing spar to fail given various failure theories while incorporating a viscoelastic model. The viscoelastic constituent model is constructed using a Prony series to characterize the compliance and relaxation functions determined experimentally from creep and relaxation tests.

\subsection{Motivation}

The focus of this research is one segment of a larger research program. The overall objective is to predict the service life of a Diamond DA20-A1 Katana operated by Ottawa Aviation Service (OAS) in Ottawa, Canada. The manufacturer states that the aircraft has an unlimited service life; however comparable aircraft in this market segment have rated service lives of 12,000 to 24,000 flight hours [2,3]. The aircraft operated by OAS are approaching 12,000 flight hours; therefore, OAS would like to 
estimate the remaining service life. The approach to predicting the service life of a glass fibre reinforced plastic (GFRP) light aircraft has two thrusts, the first is the material and structural response characterization, and the second is the modelling of the aircraft loads during operation. The focus of this thesis is on the material and structural response characterization. Figure 1.1 is an illustration of the greater project and how the components interact.

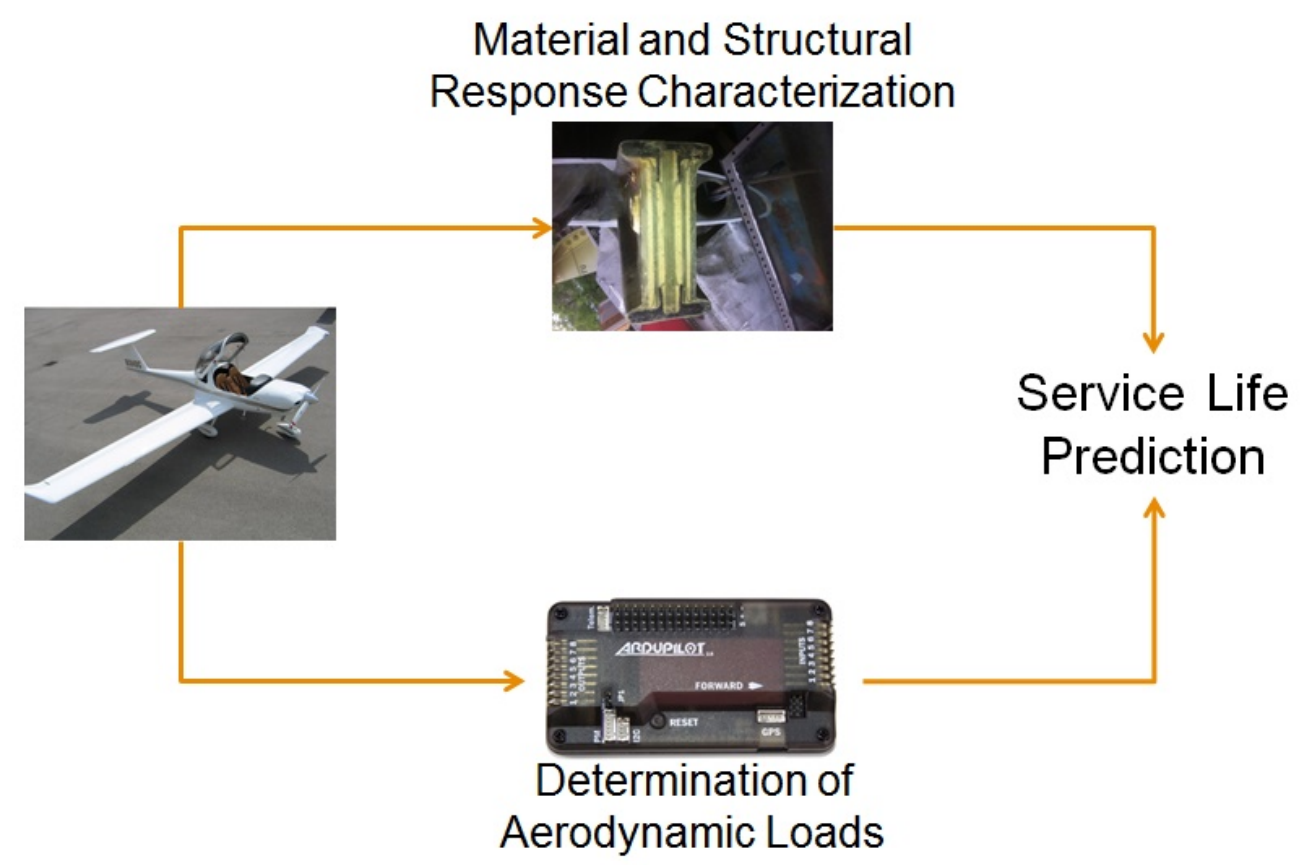

Figure 1.1: Research components of the Diamond DA-20 Katana life prediction project

\subsection{Description of the Aircraft}

The Diamond DA-20 is a two seat light, utility aircraft consisting of a polymer composite airframe conforming to both CAR 523 and FAR 23 regulations for normal, utility, aerobatic and commuter category Aeroplanes [4]. It was introduced in 1992 and was designed as a training aircraft for both military and civil applications with its most notable client being the United States Air Force. The airframe utilizes a low mounted straight wing and a T-tail empennage in conjunction with conventional flaps, ailerons, elevators and rudder as shown in Figure 1.2 and 1.3. 


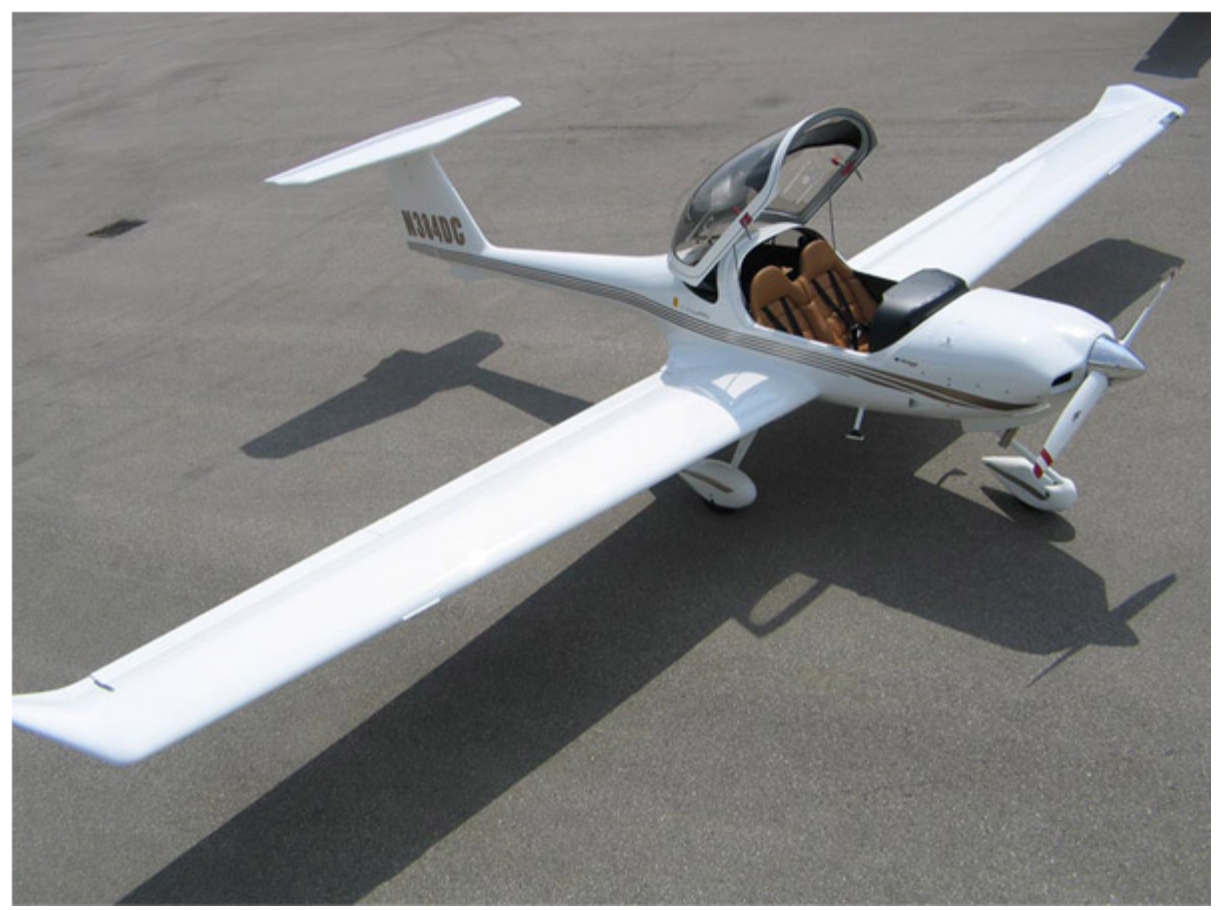

Figure 1.2: Photograph of Diamond DA-20 Katana [2]

The flight controls are mechanically actuated whereas the flaps and trim control are electrically actuated. The front mounted piston engine transfers its power to a constant speed two blade propeller to maintain optimal fuel efficiency. The intent of this thesis is to examine the effects the viscoelastic matrix will have on the service life of the airframe. The analysis will focus on the main wing spar of the aircraft as it bears the majority of the load during flight. The cantilevered I-beam spar utilizes an epoxy based matrix with glass fibre reinforcement in the web and carbon fibre reinforcement in the flanges for increased stiffness as illustrated in Figure 1.4 [2]. 


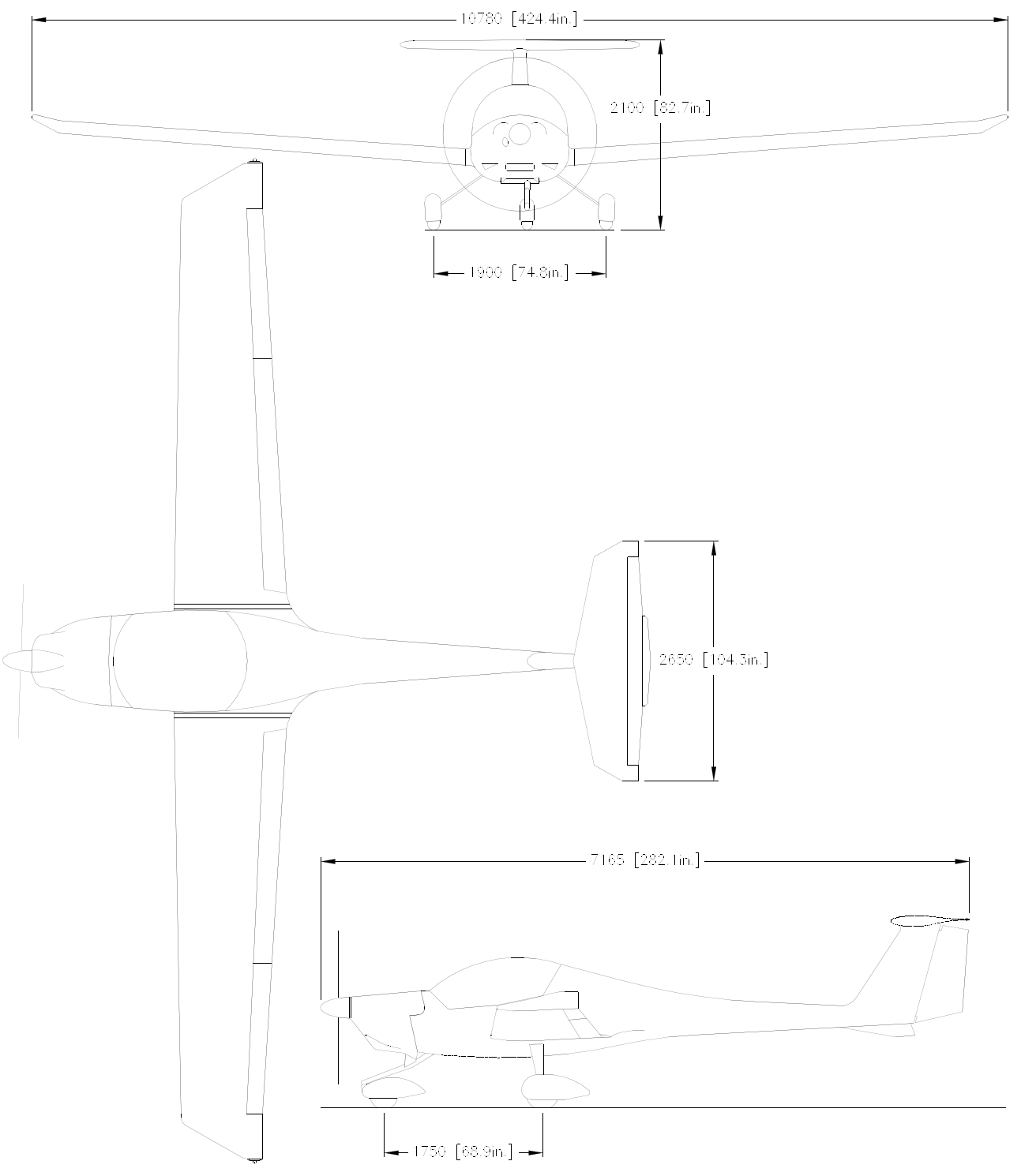

Figure 1.3: Schematic diagram of Diamond DA20-A1 Katana (mm[in]) [2] 


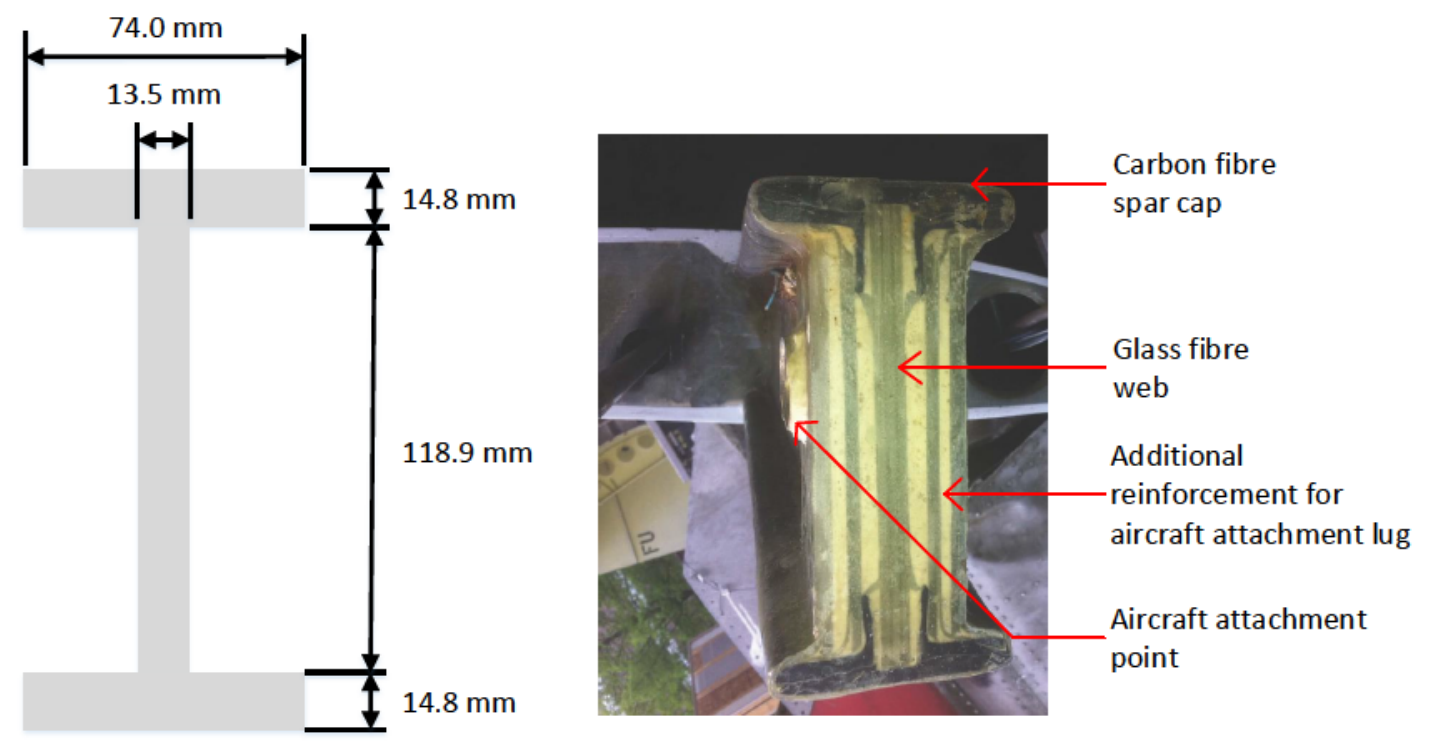

Figure 1.4: Schematic diagram and end view of the Diamond DA20-A1 wing spar

For simplicity, composite materials are typically analysed using an elastic constituent model; however, due to the molecular structure of the epoxy matrix, a viscoelastic model is more accurate as these materials tend to dissipate energy and have a memory effect. As a result of these properties, the stress within the material will accumulate over time which has the effect of lowering the failure condition of the wing spar. Before the response of the wing spar can be predicted, a variety of topics must be examined and combined. Figure 1.5 is a flow chart outlining the topics to be examined and how they are interrelated in the life prediction process. 


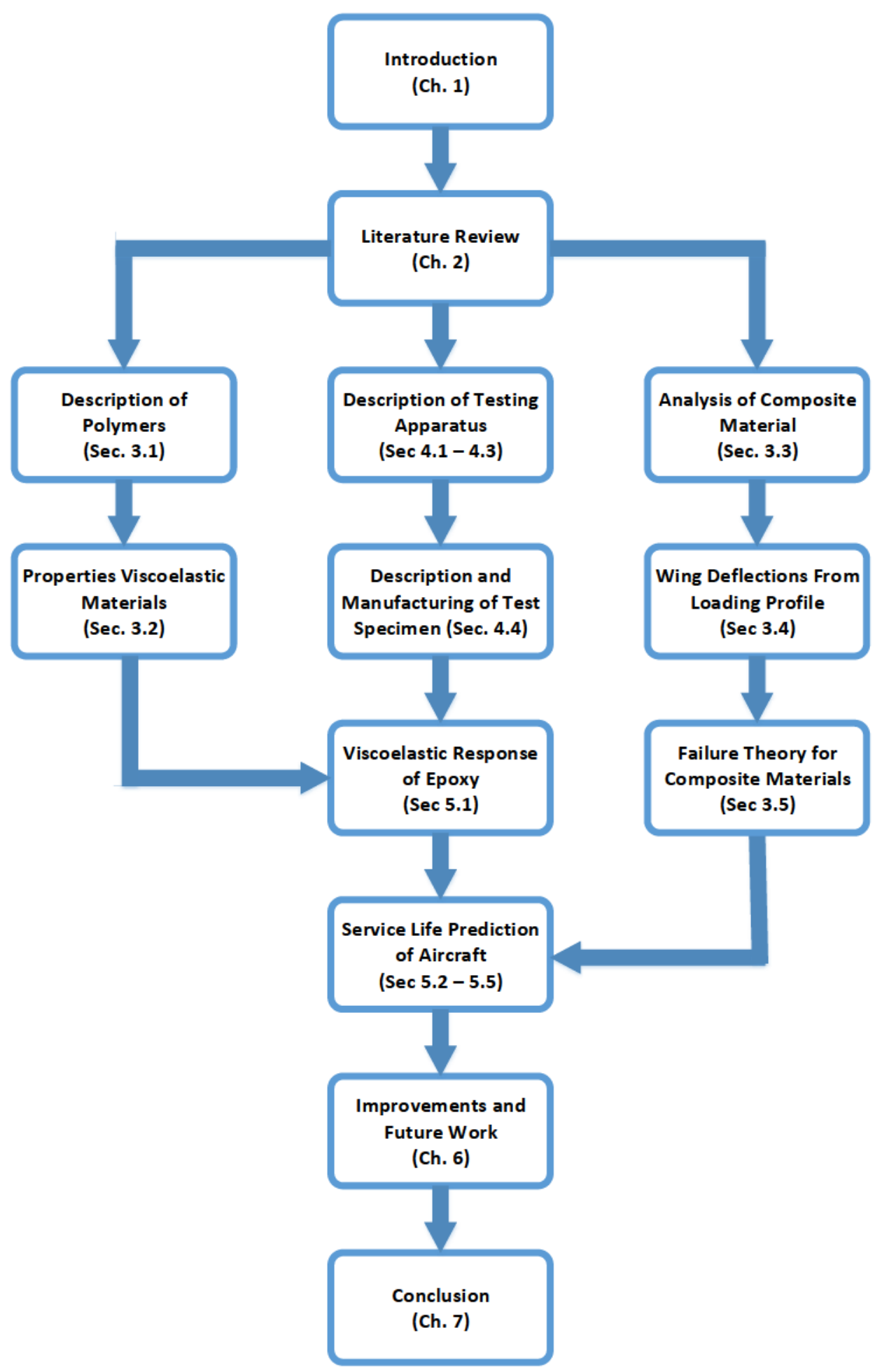

Figure 1.5: Flow chart depicting the interrelationship between each topic 


\section{Chapter 2}

\section{Review of Composite Material use the Aviation Industry}

With the rising cost of energy, the push for lighter and more efficient aircraft has been a key issue for manufacturers. One method of achieving this has been the adoption of lightweight polymer matrix composite (PMC) materials which can provide high specific strength or specific stiffness $[5,6]$. In addition to the weight saving, these materials tend to be more corrosion and fatigue resistant when compared to traditional materials like aluminium [7]. Although composite materials have been used in civil aircraft for decades, they have been limited to secondary structures as their reliabilities are not as critical to safe flight. Modern aircraft such as the Bombardier C-series, the Boeing 787 or the Airbus A350 have extensive use of composite structures comprising of approximately $50 \%$ of the aircraft including the wing, fuselage and tail sections [8]. Figure 2.1 is a graph illustrating various aircraft and their use of composite materials over time. 


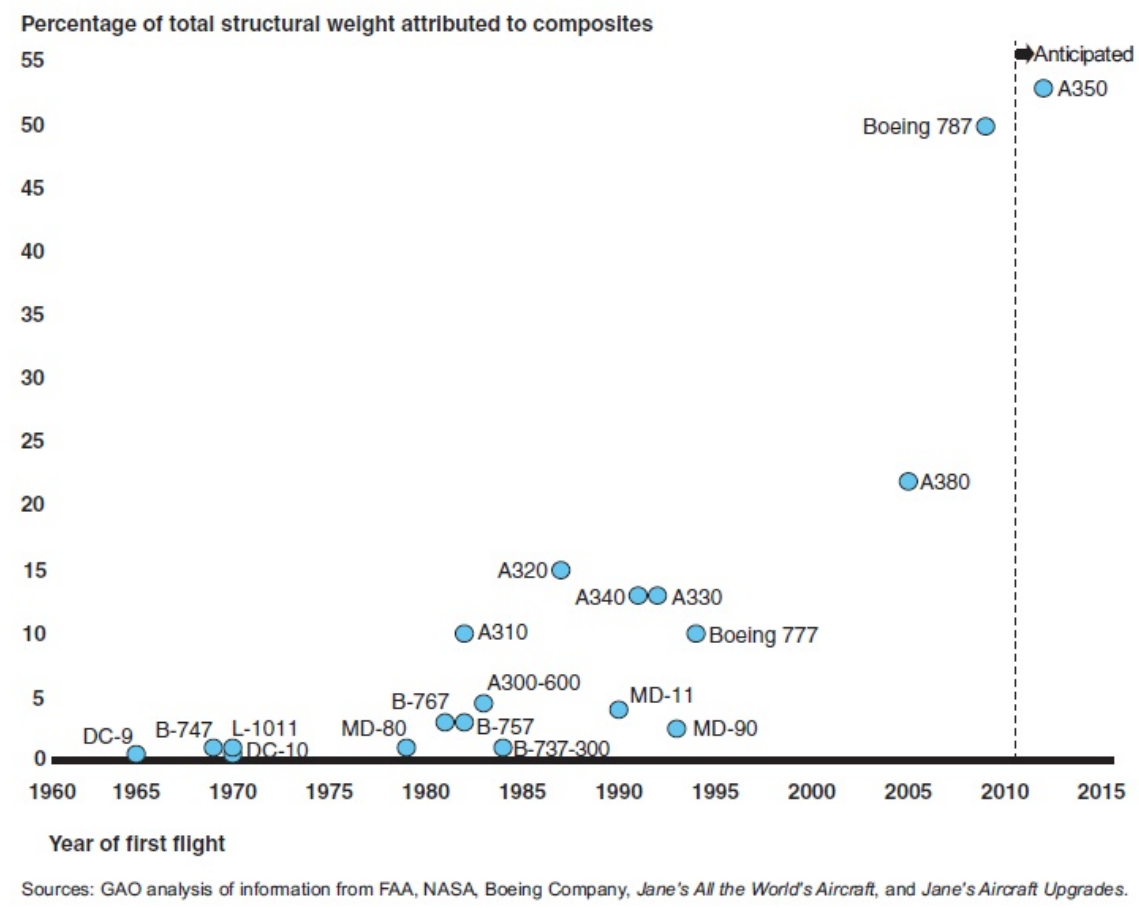

Figure 2.1: Illustration of the increased percentage of composite use in structural components for aircraft since 1960 [8]

In September of 2011, the US Government Accountability Office (GAO) issued a report on the safety of composite aircraft. This report examined the design and certification process of the Boeing 787 and came to four safety concerns regarding composite materials [8]:

1. The industry's limited experience with composite materials and how they will perform when aged or damaged. Due to limited in-service data, it was recommended that more empirical data be collected on the long term behaviour of composite materials to help create robust models or analytical methods for aircraft designers to draw from. This is especially important when designing composite aircraft as designers heavily rely on these models to predict long term behaviour, damage accumulation as well as for creating maintenance programs.

2. The technical issues around the unique properties of composite materials including the challenges in detecting and characterizing damage within the structure. This includes limited number of non-destructive tests to identify damage or the effectiveness of a repair in various layups such as variable thickness or sandwich panels. This is particularly a concern for composite repairs as they are 
susceptible to human error.

3. The lack of standardization in composite repairs. In metallic structures there are approximately a dozen common alloys used for repairs; however for composite structures there are over 60 unique materials for various types of composite structures.

4. The level of awareness of many technicians regarding composite materials. Those who have worked with metal structures for years may have limited knowledge of composite materials.

As the industry moves forward with the use of composite materials, the need to understand their long term response is crucial to determine a safe life expectancy of modern aircraft. To fully understand the concerns of the GAO, an understanding of the manufacturing process and its effect on the reliability of composite materials is required.

\subsection{Manufacturing of Composite Materials}

The manufacturing process used to construct the composite components will determine the final characteristics and reliability of the structure. Unlike isotropic metal structures where properties can be assumed consistent through the material, composite structures are composed of multiple materials bonded together. As a result, manufacturing defects such as an improper cure, voids and small deviations from the design can have a drastic outcome on the final characteristics. This however can be used to the designer's advantage as component reliability and performance are now directly coupled to the cost of manufacturing [9]. For instance, a non-critical part such as a door handle may use a cheaper manufacturing technique such as a wet-layup or vacuum bagging where a critical component such as the wing would use more sophisticated techniques and equipment such as prepreg material or the use of an autoclave for increased cure quality. Figure 2.2 shows the interrelation between manufacturing, performance and cost in the design process. 


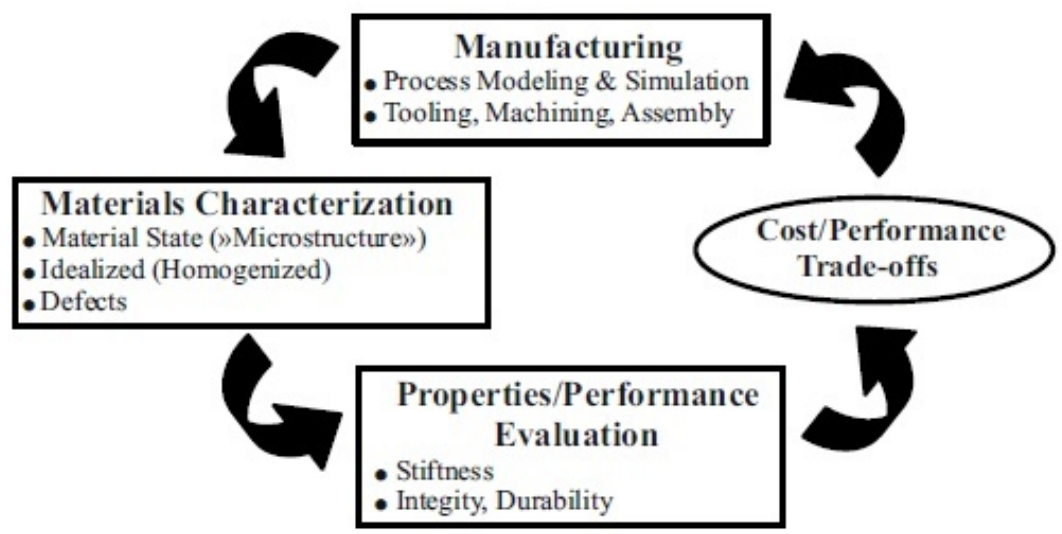

Figure 2.2: Interrelation between manufacturing, performance and cost in the design process [9]

As shown in Table 2.1, it is apparent that the manufacturing technique used can have a great influence on the quality of the part. If the quality of manufacturing is low, it can be difficult to predict its final characteristics. It is for this reason that extensive testing is performed before a composite part is certified by the aviation authorities $[7,8]$. For primary structures that affect the service life of an aircraft, high quality parts are required to eliminate manufacturing as a potential variable. The following section will outline current techniques used to evaluate the service life of composite aircraft.

\subsection{Service Life Prediction}

Limited in service experience with composites has lead to a poor ability to predict their long term response [11]. There are many phenomena which can influence the service life of composite materials. They can be fibre related, matrix related or a combination of the two. This section will outline current techniques used to determine a service life for composite structures based on damage, fatigue and viscoelasticity. 


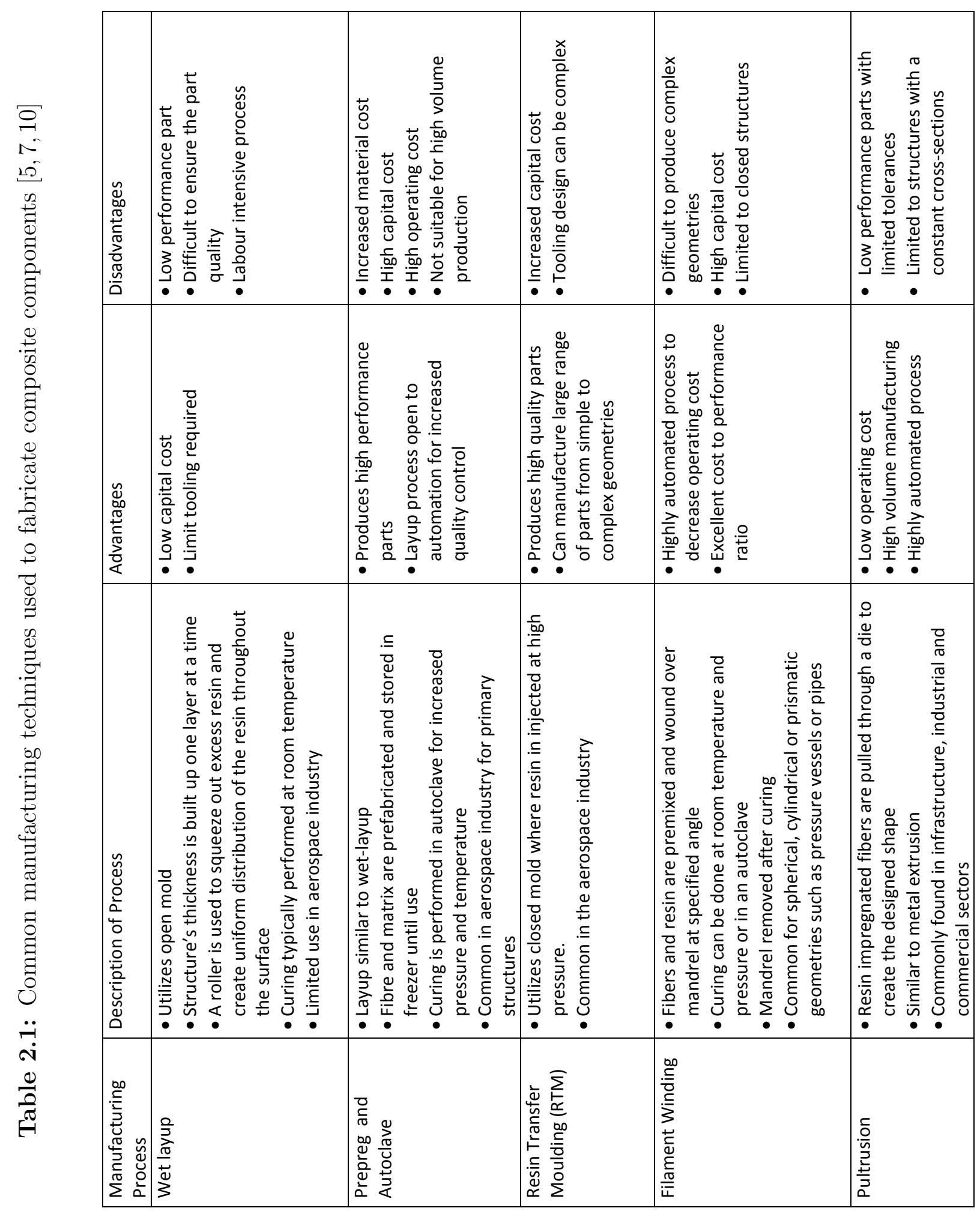




\subsubsection{Damage and its Effect on Services Life}

There are various forms of damage which may be present within a composite structure. This includes micro-cracking of the matrix, breaks in the fibre and debonding or delamination of the layers [7]. Figure 2.3 are illustrations of the various types of damage composites structures may encounter.

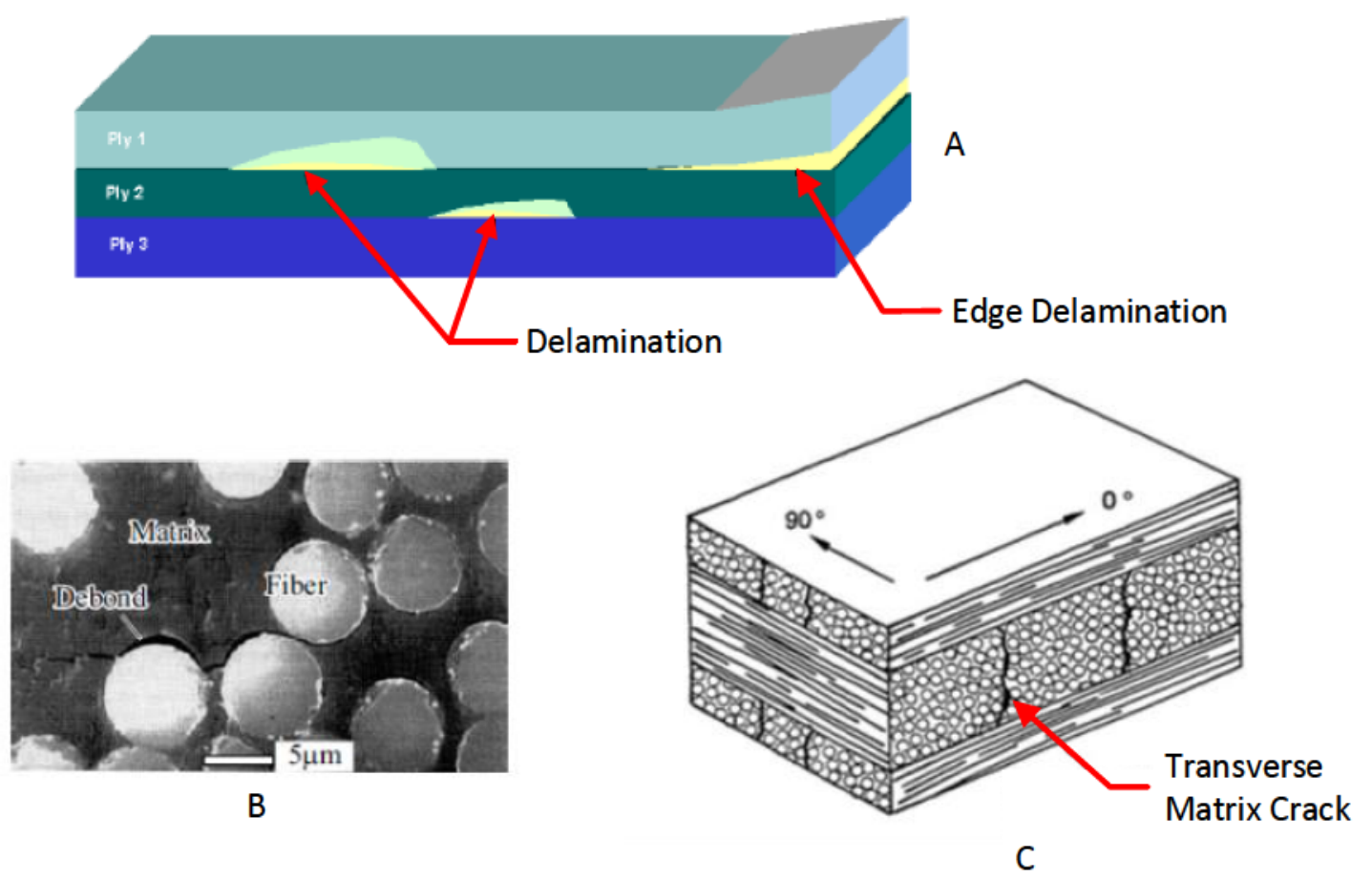

Figure 2.3: Illustration of common forms damage - A) Delamination, B) Fibre/matrix debonding, C) Matrix microcracking [7]

These are the basic types of damage and may be internal to the micro-structure. Small localized micro-damage may not significantly affect the performance of the structure; however its accumulation will lead to large and visible damage in the structure such as cracks and tears [7]. A common cause for damage is from an impact on the outer surface of the structure. The impact breaks the fibre strands resulting in a reduced overall load carrying capability. In addition, this type of damage may not be visible from the surface and may require special testing procedures such as ultrasonic or $\mathrm{x}$ rays scanning which is an ongoing form of research $[5,12]$. Figure 2.4 is an illustration of various degrees of impact damage. 


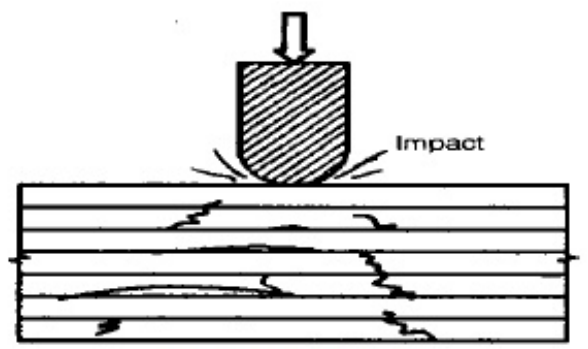

Undetectable Damage

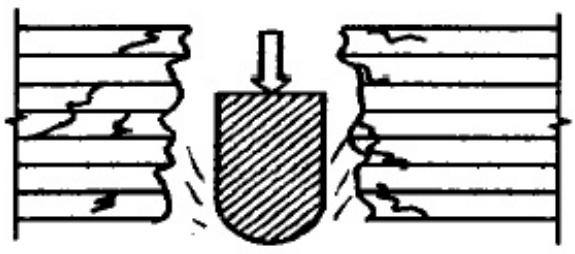

Penetrating Damage

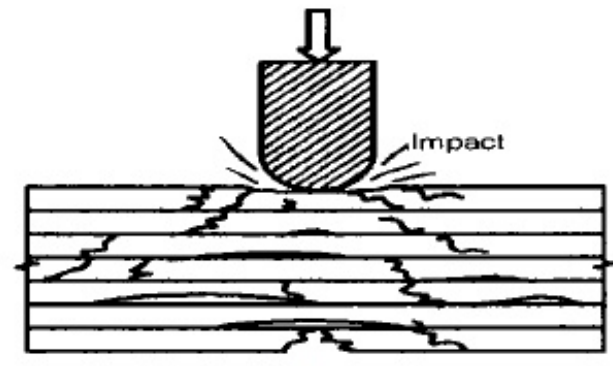

Detectable Damage

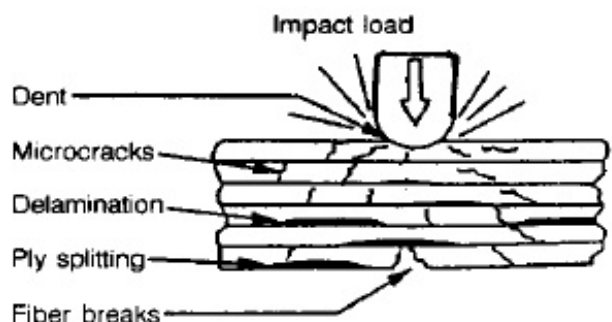

Figure 2.4: Various degrees of Impact Damage encountered by composite structures $[7]$

Currently, this type of damage is incorporated in the life prediction process using statistical analysis based on what an average aircraft would experience in its lifetime [7]. In addition to this type of damage, environmental conditions such as moisture and UV rays may affect the quality of the matrix material. These are typically controlled using protective coatings; however, care must still be taken to ensure the quality of composite material [7]. The next phenomena to be examined will be the effect fatigue has on the service life of an aircraft.

\subsubsection{Fatigue and its Effect on Service Life}

Fatigue is a phenomena which can be defined as the weakening of the material as a result of repeated loading below its yield strength. The root cause is from microscopic imperfection in the crystal structure which leads to stress concentrations and microcracking [13-15]. Due to the extensive use of metals in modern society, mathematical models to represent fatigue's effect on metals has been the focus of ongoing research as direct testing of every structure is uneconomic and time consuming for an accurate life prediction. Since the use of composite materials are is relatively new compared 
to the use of metals, there are limited theories to properly represent its effects on composites as many mechanisms the tend to be more complex $[6,7,14,16]$. Figure 2.5 is a illustration comparing the difference in results between a metal and a composite fatigue test on a notched specimen.
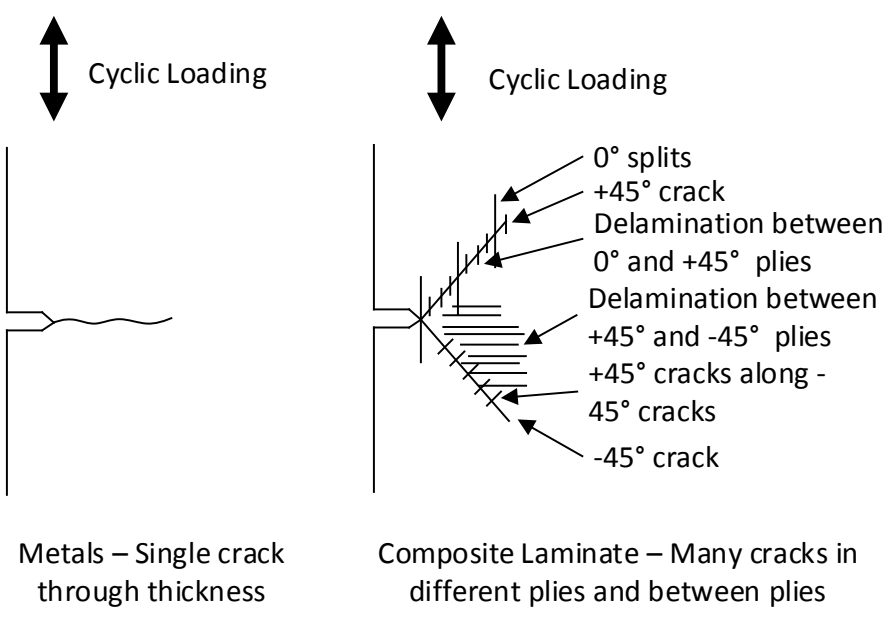

Figure 2.5: Comparison showing the complex nature of fatigue damage in composites compare to that found in metals during a notch test [7]

Composite structures are constructed of multiple materials and can be highly anisotropic which introduce many variables [13]. For instance, multi-directional lamina resist fatigue crack growth owing to the support provided by adjacent plies with fibre orientations opposite to the direction of crack growth $[7,14]$. Current methods of characterizing fatigue in composite structures are through direct modulus degradation, determination of a fatigue modulus, residual strength, fatigue damage accumulation or through energy methods. Each of these methods have advantages and disadvantages and are typically selected based on the load type and frequency [6,16].

\subsubsection{Viscoelasticity and its Effect on Services Life}

Viscoelastic phenomena such as creep and relaxation are a concern for long term response of the material. Viscoelastic materials have relatively low operating temperatures and are susceptible to thermal ageing which can be a concern for high speed aircraft [17]. In addition, viscoelastic materials exhibit a memory effect in which the previous loading will affect the current stress state of the material [18]. Until recently, 
research into viscoelasticity and its effect on service life have been limited due to its complex nature and the moderate use of composite materials in aircraft. In addition to the effect viscoelastic materials have on service life, it has been found that the load history has an effect on flutter and this is currently an active research area [19-21].

\subsection{Testing Methods for Viscoelastic Materials}

Currently there are multiple tests to characterize the response of viscoelastic material. Common tests to characterize the mechanical properties of viscoelastic materials are through direct tension tests, dynamic mechanical analysis (DMA) or through broadband viscoelastic spectroscopy (BVS) [7,22]. The uni-directional tension test is the most basic and cost effective way to determine the long term response of viscoelastic material as it directly measures the creep and relaxation functions. DMA test equipment applies a cyclic load and the response of the material is measured using sensitive instruments. Viscoelastic properties are measured and determined based on the phase difference between the load application and the material's response [7]. BVS is a test similar to a DMA test; however, the load is applied to the gripping mechanism through a magnetic field and the response of the material is measured using laser spectrometry. This allows for more precise measurements between the load application and the material's response which allows for higher frequency tests [22]. An illustration of these types of test equipment are found in Figure 2.6. 


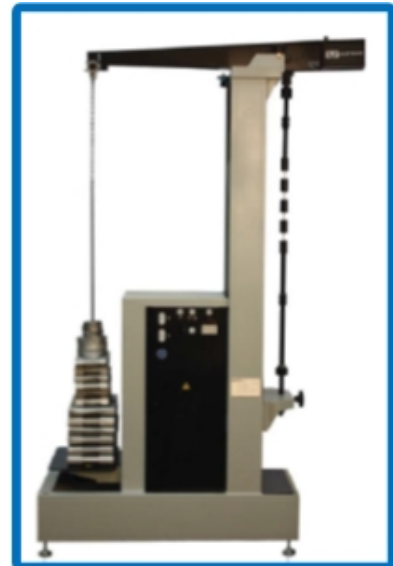

(A)

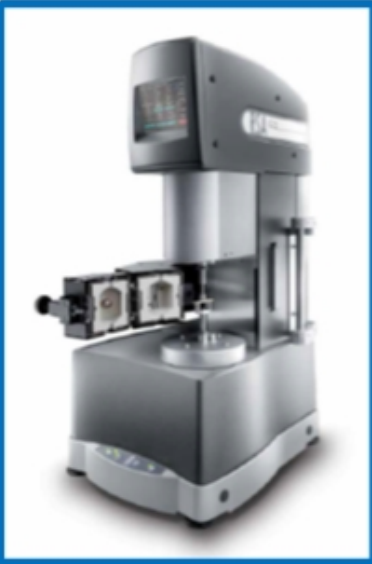

(B)

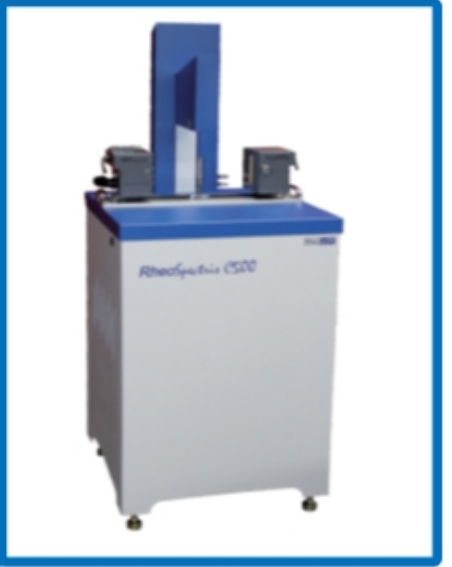

(C)

Figure 2.6: Various viscoelastic testing apparatus: (A) - Instatron Stress Rupture Testing System - Model C, (B) - TA Instruments RSA-G2 Solid Analyser, (C)

- Rheolution Instruments Hyper Frequency Viscoelastic Spectroscopy 


\section{Chapter 3}

\section{Theory of Viscoelastic and Composite Materials}

This chapter provides the theory behind viscoelasticity and how it relates to polymers as well as the analysis of composite materials. This includes the material's response in bending, failure conditions as well as an analysis of spar's resistance to buckling.

\subsection{Characteristics of Polymers}

There are many differences between the molecular structures of metals and polymers which are responsible for the differences in their mechanical response. Secondary forces, such as covalent bonds and van der Waals forces, have a significant effect on the bulk response of the material [18]. Polymers are typically long chains of organic molecules whose repeating structure may amount to a molecular mass that approaches infinity. They are typically classified as either a plastic, elastomer or fibre as shown in Figure 3.1 [18,23].

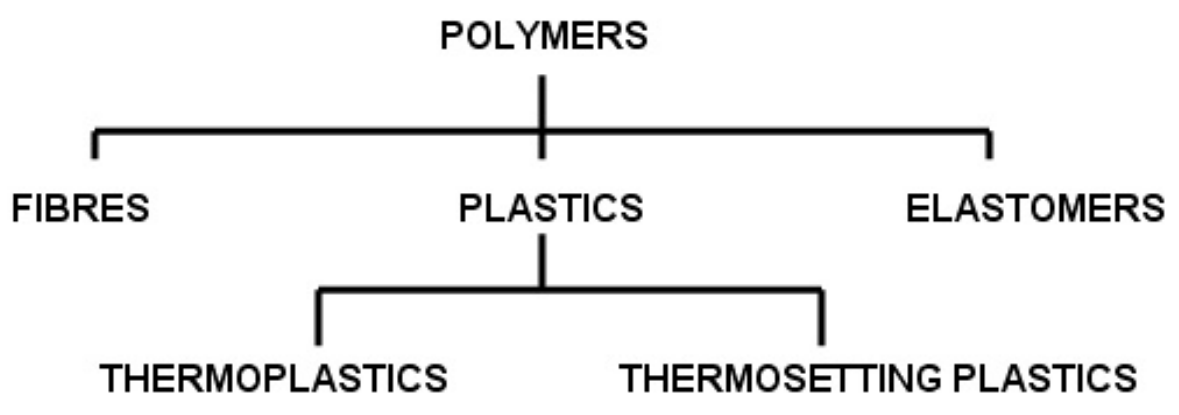

Figure 3.1: Simplified classification scheme of engineering polymers [23] 
PMC materials are typically manufactured using either thermoplastic or thermosetting polymers and will be the focus of this section. In both cases the intrachain molecules are held together by covalent bonds; however it is the interchain bonds which lead to their different characteristics. In thermoplastic polymers, the individual chains are held together solely by van der Waals forces which is why they are also referred to as linear polymers. The chains of thermosetting or cross-linked polymers are attached by both covalent and van der Waals bonds. Figure 3.2 depicts a generalized micro-structure for engineering plastics.

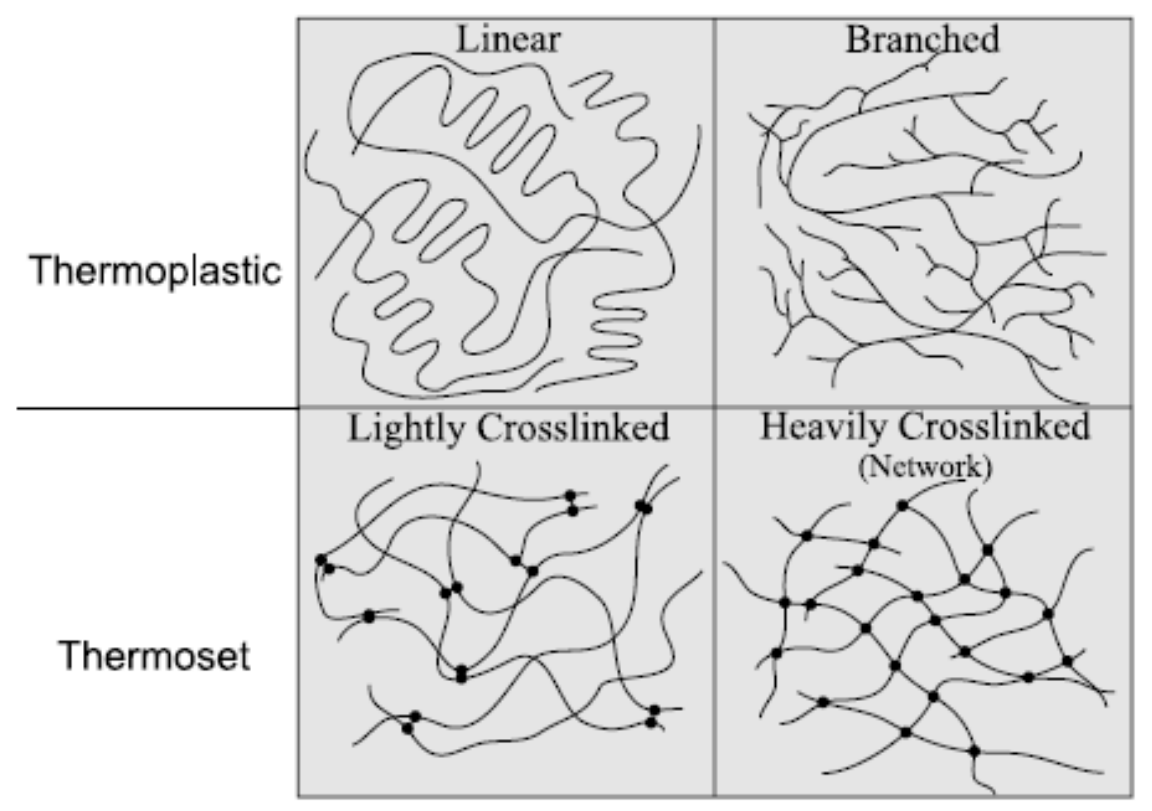

Figure 3.2: Generalized micro-structure comparing thermoplastic and thermoset polymers $[18]$

A key characteristic of polymers is their ability to dissipate energy through their molecular chains which produce a phase delay in their mechanical response. The following section will briefly compare the viscoelastic nature of thermoplastics and thermosets; however the latter will be the primary focus as DA-20 uses an epoxy based composite, a cross-linked thermosetting polymer. 


\subsection{Properties of Viscoelastic Materials}

In addition to polymers, viscoelasticity can be observed in a vast range of materials as illustrated Figure 3.3.

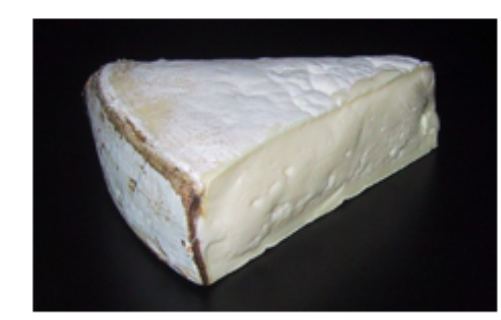

Food: Cheese

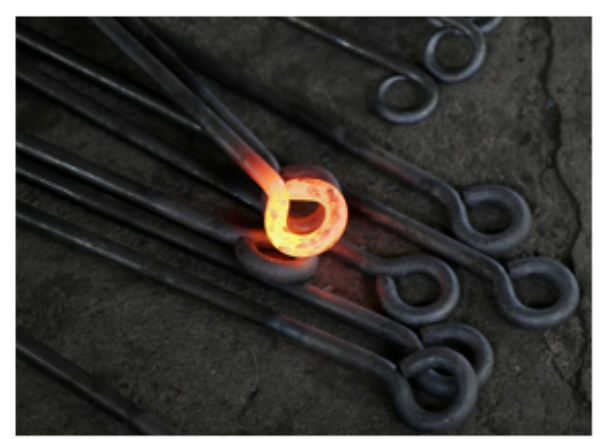

High temperature metals

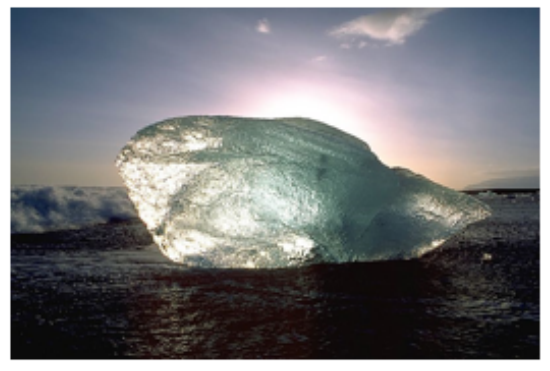

Ice

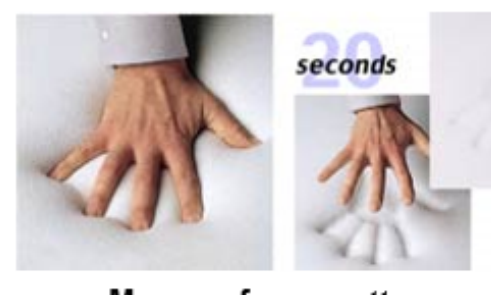

Memory foam mattress

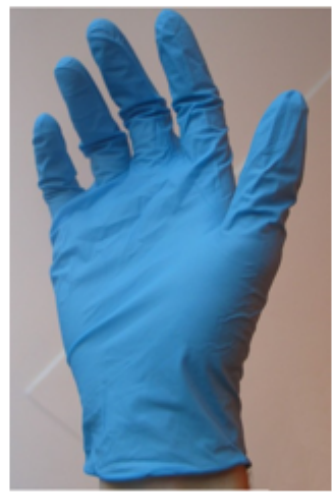

Polymers

Figure 3.3: Various examples of viscoelastic materials common to our society

These materials exhibit three common phenomena; creep, recovery and stress relaxation. Creep is characterized as the slow deformation of a solid under a constant load. A practical example of this would be the slow stretching of a filled plastic bag. This phenomena can be broken into four categories; the initial strain and the primary, secondary and tertiary stages of creep [14]. Recovery is the reverse of creep and is described as the gradual reversal of the creep strain once the load is removed. As a result of cross-linking bonds between chains, an ideal thermosetting polymer will return to its original state after being unloaded; however this typically occurs at significantly slower rate than the creep deformation $[1,18]$. A comparison of the creep and recrovery response of thermoplastic and thermosetting polymers are illustrated in Figures 3.4 and 3.5 . 

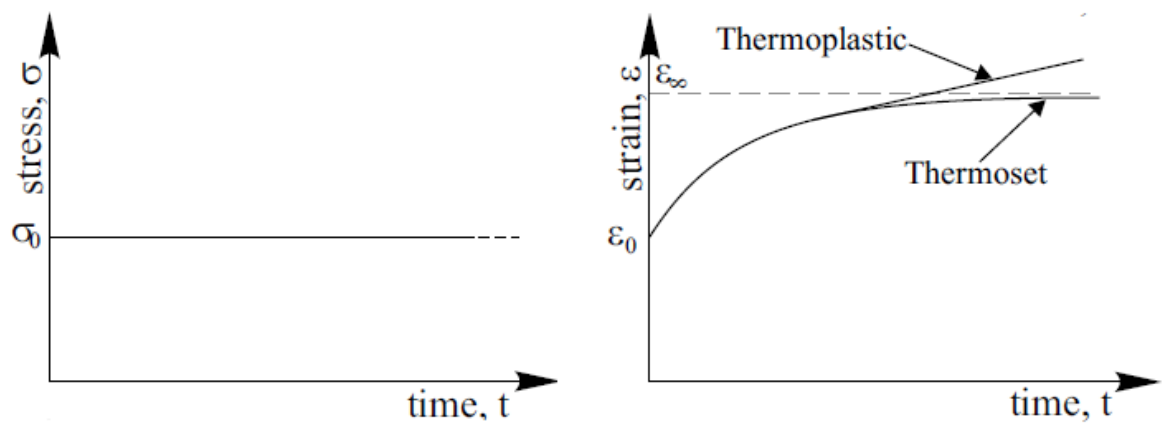

Figure 3.4: Creep response of thermosetting and thermoplastic polymers given constant stress, $\sigma_{o}[18]$
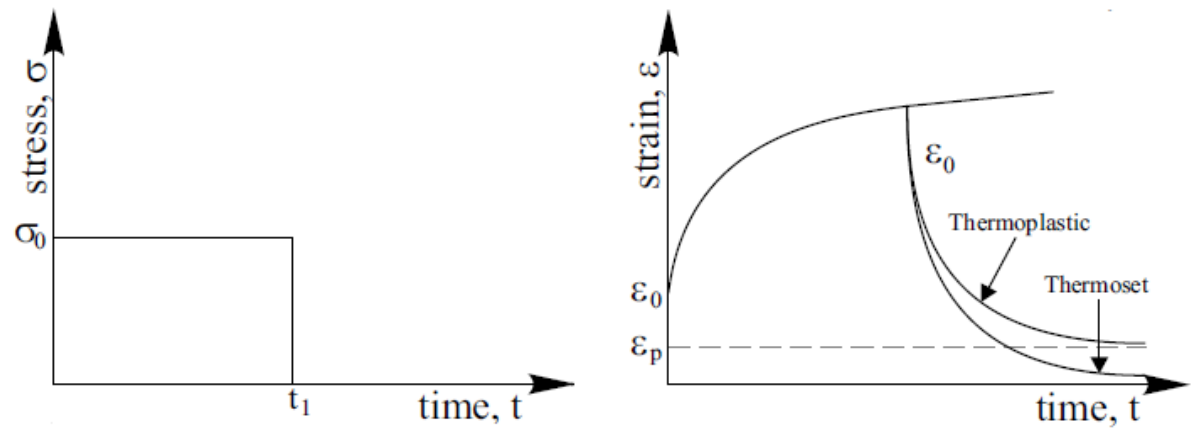

Figure 3.5: Creep and recovery response of thermosetting and thermoplastic polymers given constant stress, $\sigma_{o}[18]$

The final phenomena is known as stress relaxation and is a material's tendency to decrease stress given a constant displacement over a period of time [14]. This long term characteristic can be seen in many common objects such as elastic bands or rubber washers. Unlike thermoplastic materials which will completely relax, the amount of stress within a thermosetting polymers will approach to a steady state value as depicted in Figure 3.6 [18]. 

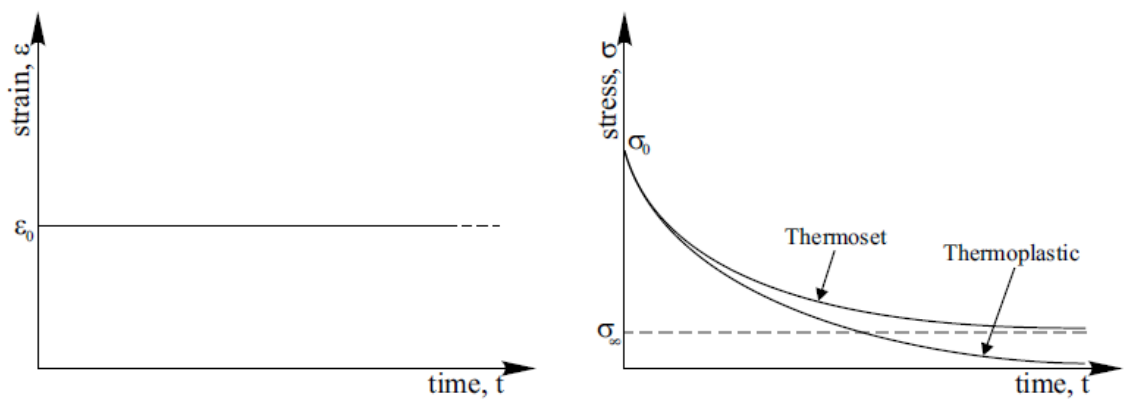

Figure 3.6: Relaxation curves for thermosetting and thermoplastic polymers given a constant strain, $\varepsilon_{o}[18]$

Although creep and relaxation are a result of the same molecular phenomena, its effect of stiffness has been shown to occur at different rates in areas of high rate change. It has been shown that the relaxation response approaches equilibrium faster than that seen by the creep response $[18,24]$. The simplest representation of these materials would be to combine an elastic Hookean solid and a viscous Newtonian fluid. These are typically modelled using a spring for elastic deformation and a dashpot to symbolize the slow gradual deformation over time. Mathematically, the materials are represented as:

$$
\begin{gathered}
\sigma_{\text {elastic }}=E \varepsilon \\
\sigma_{\text {viscous }}=\eta \frac{\partial \varepsilon}{\partial t}
\end{gathered}
$$

where $E, \varepsilon$ and $\eta$ are the elastic modulus, strain and damping coefficient. Viscoelastic models are typically constructed by combining these components and are represented graphically using two simple models; the Maxwell and Kelvin models which are shown in Figure 3.7 [1]. 

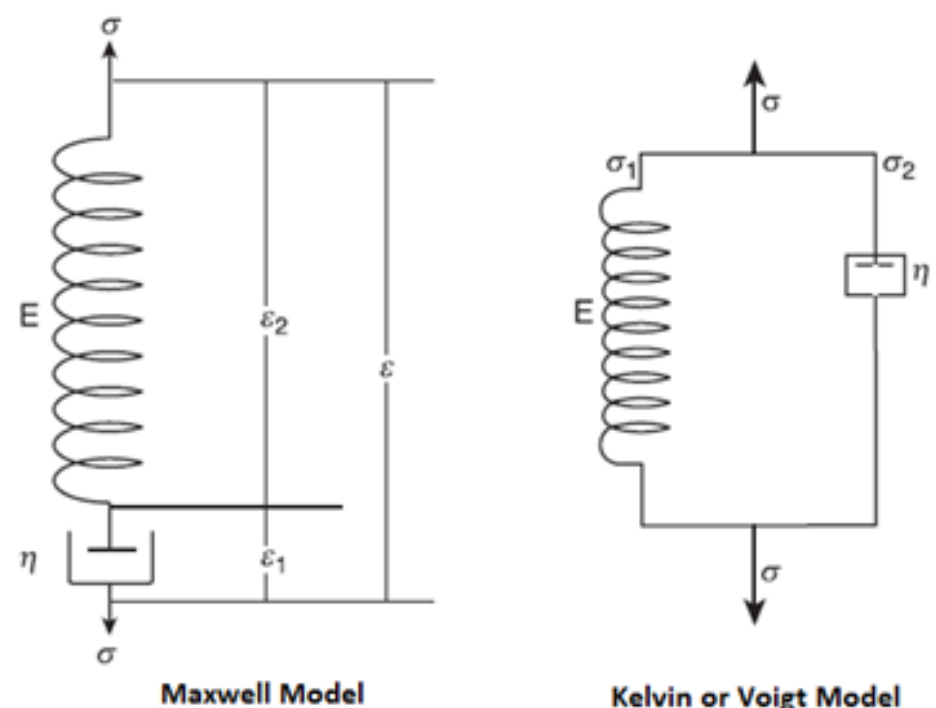

Kelvin or Voigt Model

Figure 3.7: Illustration of the Maxwell and Kelvint viscoelastic models [1]

where $\varepsilon_{1}$ and $\varepsilon_{2}$ represent the strain in the spring and the dashpot for the Maxwell model, and $\sigma_{1}$ and $\sigma_{2}$ represent the stress in the spring and the dashpot for the Kelvin model. Due to their simplicity, these models are not capable of properly characterizing a real viscoelastic material since the Maxwell model is unable to model creep and recovery and the Kelvin model is unable to model relaxation. As a result, more sophisticated models are a combination of these and they are typically empirically calculated to fit experimental data using the Integral Constitutive Law [18].

$$
\begin{aligned}
& \varepsilon(t)=\sigma_{o} J(t)+\int_{0}^{t} J(t-\xi) \frac{\partial \sigma(\xi)}{\partial \xi} d \xi \\
& \sigma(t)=\varepsilon_{o} Y(t)+\int_{0}^{t} Y(t-\xi) \frac{\partial \varepsilon(\xi)}{\partial \xi} d \xi
\end{aligned}
$$

To determine the relaxation $J(t)$ and compliance functions $Y(t)$, both creep and stress relaxation tests must be performed.

\subsubsection{Experimentally Determined Creep and Relaxation Characteristics}

As described in Section 2.3, the most direct means of determining a material's viscoelastic properties are by performing stress relaxation and creep deformation tests. 
These tests are typically done in a uniaxial direction and the variation in the stiffness is governed by a Prony series characterized in Equation 3.5 [18,25]. A Prony series can be classified as the summation of many exponential terms and as a result is a convenient way to model the interactions of a system of springs and dashpots.

$$
Y(t)=E_{0}\left[1-\sum_{i=1}^{n} E_{i}\left(1-e^{-t / \alpha_{i}}\right)\right]
$$

where $E_{0}$ is the initial elastic modulus, $E_{i}$ is a constant for the Prony series, and $\alpha_{i}$ is the relaxation time. Similar equations can be employed to characterize the compliance function $J(t)$. Figure 3.8 is an example of a Prony series fit to experimental data.

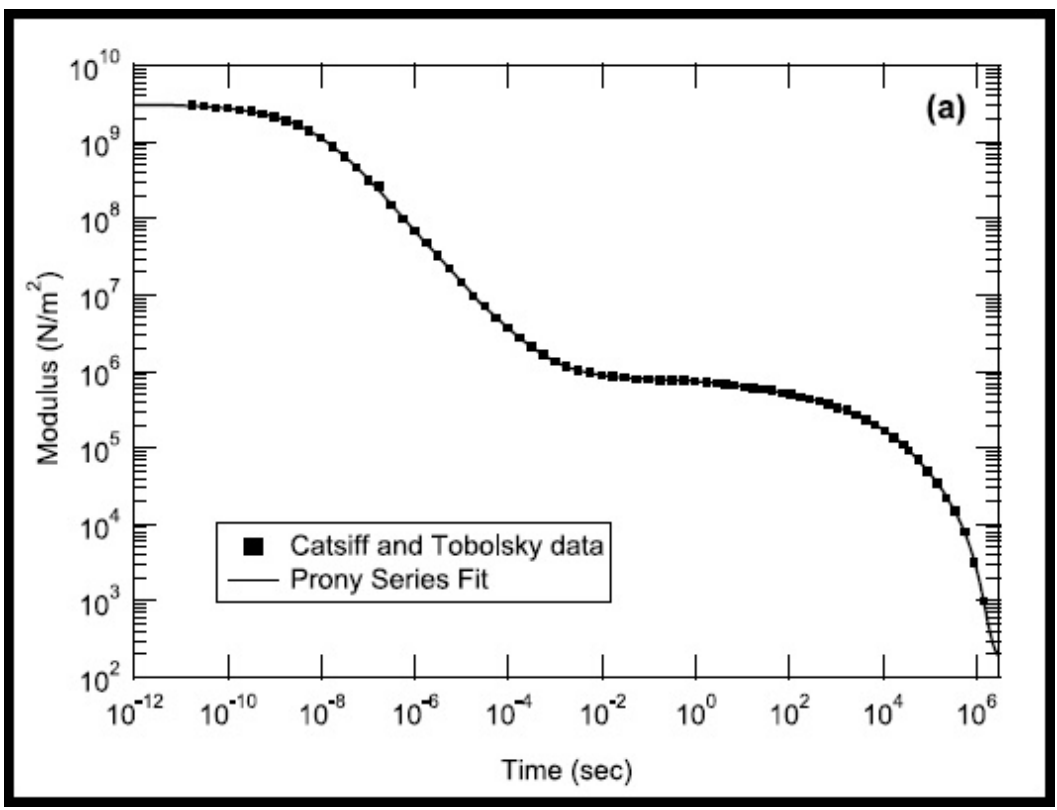

Figure 3.8: Measured relaxation function for polyisobutylene and the series expansion fit (Original data from Tolbolsky (1972) and Catsiff and Tobolsky (1955)) [18]

Since these tests may take an extended period of time at room temperature, the tests are normally performed at an elevated temperature and a time-temperature correlation is used.

\subsubsection{Effect of Temperature}

Temperature has a dramatic effect on the viscoelastic characteristics and requires analysis to be done over a wide range of temperatures $[18,26,27]$. The mechanical 
response of these materials can be classified into three main categories based on their temperature with respect to the glass transition temperature. Glass transition is the point where viscoelastic materials observe a rapid change in stiffness as depicted in Figure 3.9. For temperatures below the glass transition point the material is classified as glassy and for temperatures above this point they are classified as rubbery. For temperatures around this point the material is classified as in transition. Figure 3.9 is an illustration of the material's stiffness with respect to temperature for various polymer types. It is important to note that the flow regime will not occur in cross linked polymers such as epoxy.

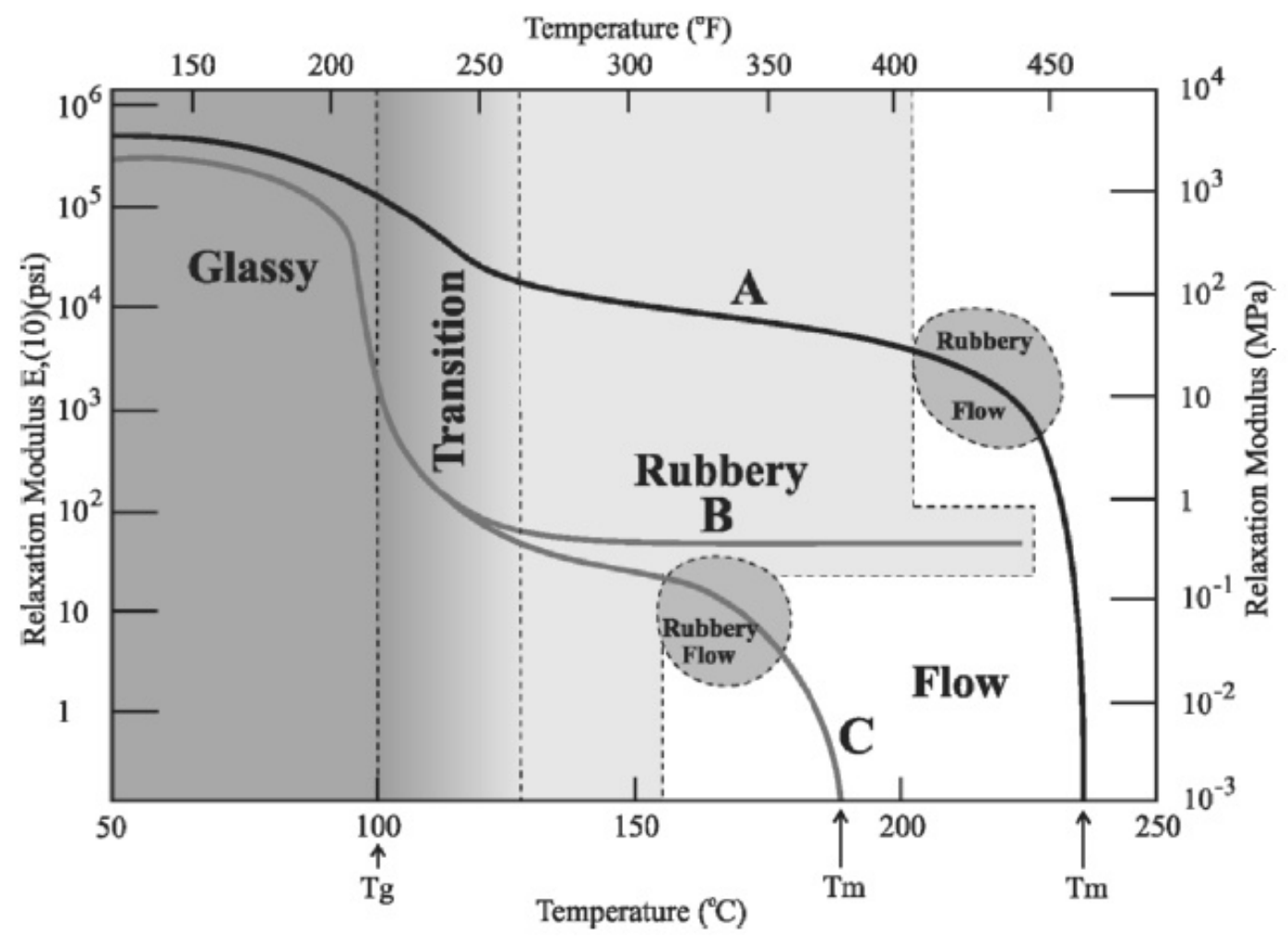

Figure 3.9: Stiffness with respect to temperature for various types of polymers: A) Crystalline polystyrene, B) Cross-linked polystyrene, C) Amorphous polystyrene [18]

Temperature also has the effect of varying the viscoelastic response time depending on the direction of the temperature change. As the temperature increases the response time decreases and as temperature decreases the response time increases [18,26,27]. 
This characteristic can be experimentally exploited to help observe the material response in a realistic time frame. For instance, to observe rapid short term behaviour one could reduce the test temperature and to observe long term behaviour one could increase the temperature. For polymers with simple micro structures such as an amorphous thermoset, it can be assumed that the material is thermorheological and the variation is seen as a horizontal shift of the logarithmic curve as shown in Figure 3.10 and is represented using Equation 3.6 [18,24].

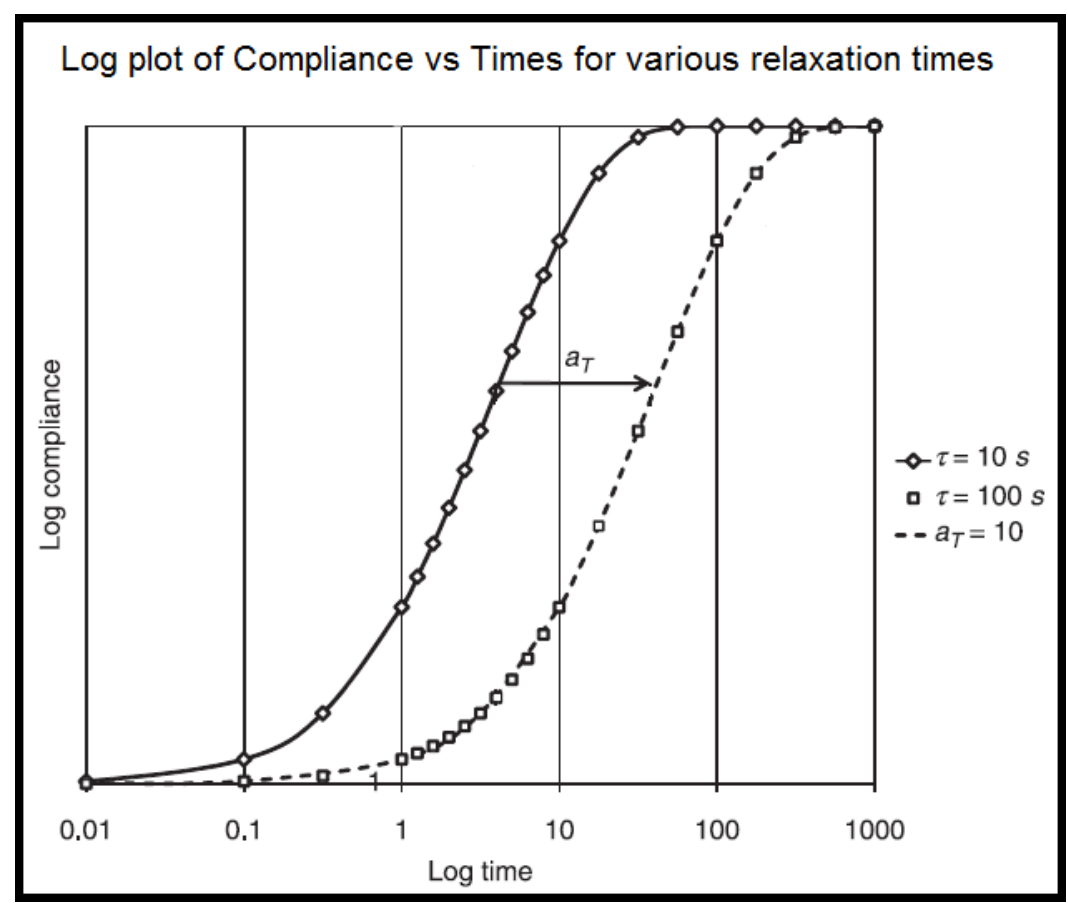

Figure 3.10: A general example of the effect of decreasing the relaxation time by increasing the test temperature $[1,24]$

$$
\log a_{t}=\log \tau(T)-\log \tau\left(T_{r e f}\right)
$$

where $a_{t}, \tau, T$ and $T_{\text {ref }}$ are the change in relaxation time, the relaxation time, the actual temperature and reference temperature respectively. If the relaxation time takes an Arrhenius form shown in Equation 3.7, the shift factor $\log a_{t}$ is governed by Equation 3.8 [24].

$$
\tau(T)=\tau_{o} \exp \left(\frac{E_{a}}{R T}\right)
$$




$$
\log a_{t}=\frac{E_{a}}{2.303 R}\left(\frac{1}{T}-\frac{1}{T_{r e f}}\right)
$$

where $E_{a}$ and $R$ are the material's activation energy and the universal gas constant respectively. For temperature above glass transition, researchers feel that this shift factor is governed by other principles and that other equations such as William Landel Ferry equation, shown in Equation 3.9, better represent this shift [1,24].

$$
\log a_{t}=\frac{-C_{1}\left(T-T_{r e f}\right)}{C_{2}+\left(T-T_{r e f}\right)}
$$

where $C_{1}$ and $C_{2}$ are empirically calculated constants. It has been found that if the reference temperature is the glass transition temperature of the material, $C_{1}$ and $C_{2}$ are universally assumed to be $17.4 \mathrm{~K}$ and $51.6 \mathrm{~K}$ respectively [1,24]. Substituting these values yields Equation 3.10.

$$
\log a_{t}=\frac{-17.4\left(T-T_{g}\right)}{51.6+\left(T-T_{g}\right)}
$$

To determine the viscoelastic response of the material throughout the lifetime of the aircraft, master curves must be generated for various reference temperatures using the principal of time-temperature shifting.

\subsubsection{Generation of Master Curves}

To generate the set of master curves that govern the response of the material, an initial curve is generated at room temperature. To create the initial curve, multiple tests are performed over a range of temperatures to collect modulus data as each will better capture the material response for the given temperature. Ideally, test time should be kept to a minimum to reduce the adverse effect of elevated temperatures such as the thermal oxidation of the polymer chains as illustrated in Figure 3.11. 


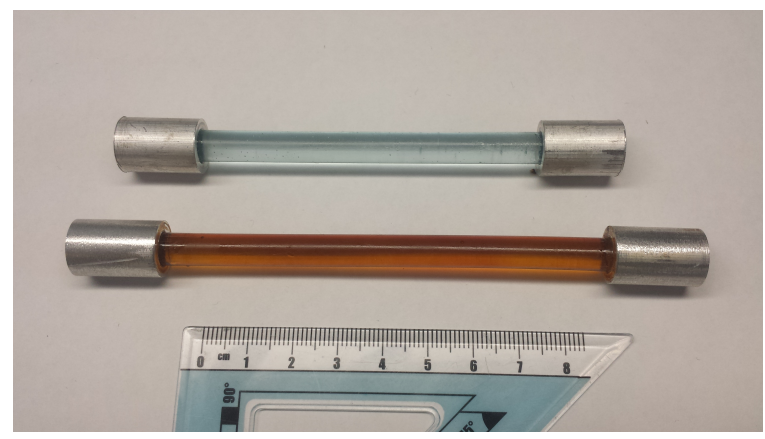

Figure 3.11: Illustration of thermal oxidation to a test specimen when exposed to high temperature for an extended period of time: Top - New specimen, Bottom - Thermally oxidized specimen

This data is then shifted to the appropriate reference temperature to generate the master curve as shown in Figure 3.12. Once the reference curve has been created it can be shifted over a wide range of temperatures to generate a set of curves that define the viscoelastic response of the material.

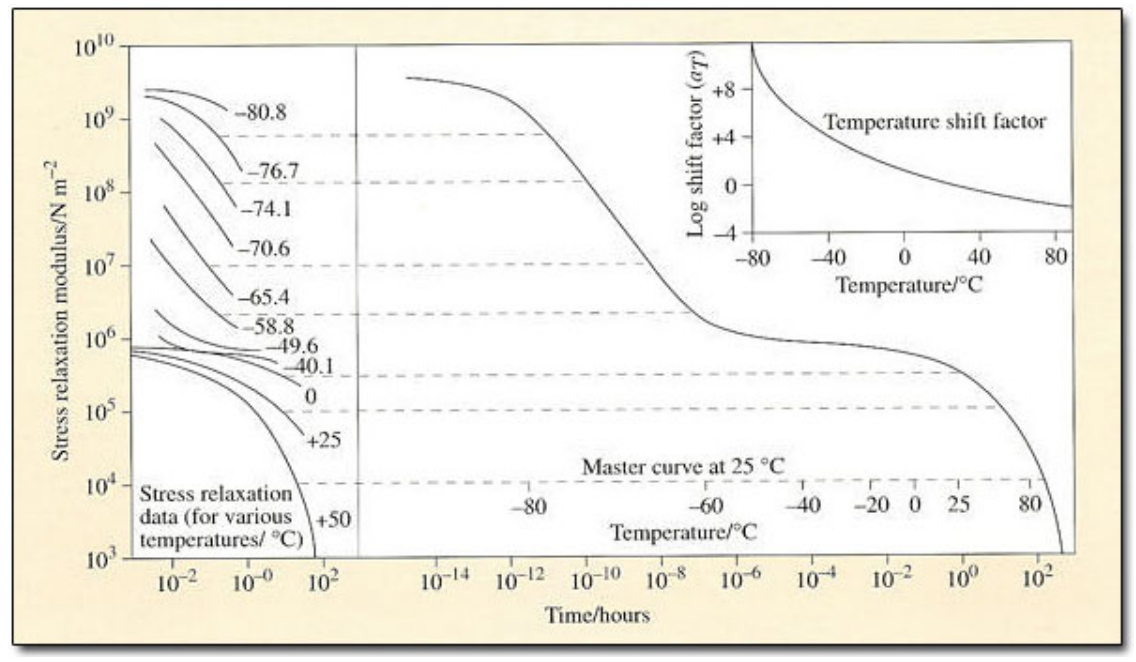

Figure 3.12: Hypothetical construction of a viscoelastic master curve for PIB at $25^{\circ} \mathrm{C}$ reference temperature [28]

The final viscoelastic property to be considered is the time dependent nature of the Poisson's ratio. 


\subsubsection{Time Dependent Nature of Poisson's Ratio}

For elastic materials the coupling modulus $E_{12}$ can be determined using the Poisson's ratio $\nu_{12}$. However due to the properties of viscoelastic materials, Poisson's ratio will vary with time as shown in Figure 3.13 which compares Poisson's ratio and compliance over time at a constant temperature [29].

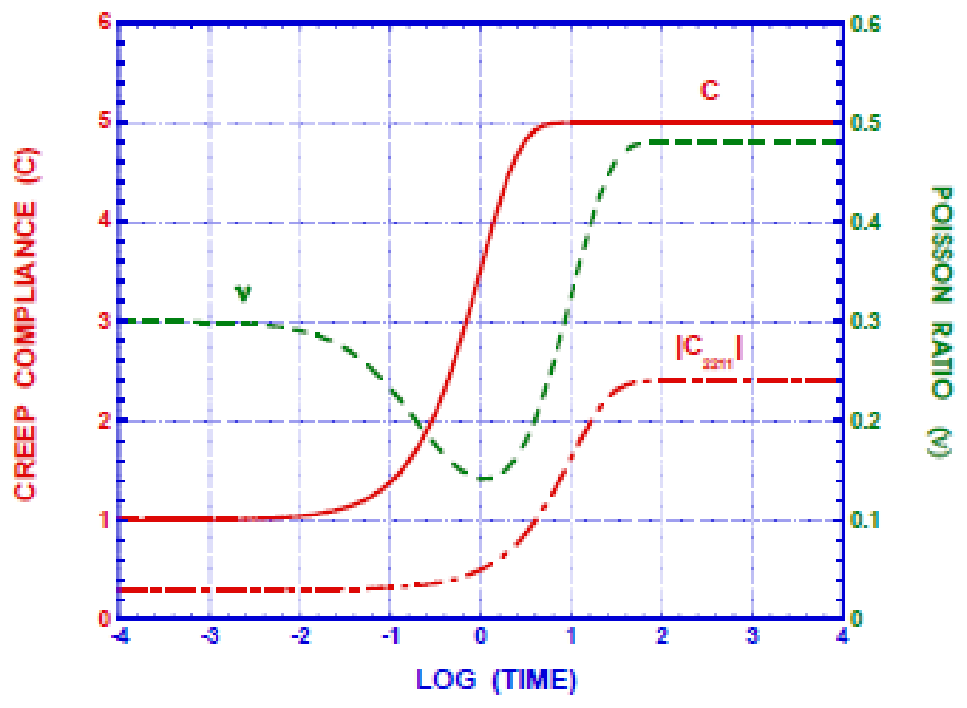

Figure 3.13: Comparison of the compliance and the Poisson's ratio for a viscoelastic material against the log of time [29]

To properly capture the time dependant nature of the coupling moduli in viscoelastic materials, one requires at least two independent moduli such as the Young's modulus, shear modulus or bulk modulus [18]. Typically this can be a cumbersome task and only one of these are known at any given time. As a result, there are three assumptions that are used in practice to capture this time dependency. These assumptions are as follows [18]:

1. The material is incompressible which can be assured by setting the Poisson's ratio to 0.5 . This leads to a infinitely large bulk moduli in both the time and Laplace domain as show in Equation 3.11.

$$
K(t)=\bar{K}(s)=\infty
$$


Typically this assumption is used for materials in the rubbery regime above it's glass transition temperature.

2. Strain dilations are assumed to be elastic which is assured by allowing the bulk modulus to remain constant. This is typically a good assumption since there is much less variation in the viscoelastic bulk moduli with time and temperature. Unfortunately with this assumption the Poisson's ratio retains its time dependant nature.

3. The bulk and shear moduli vary in a synchronous manner. Using this assumption it can be stated that the bulk modulus is always proportional to the shear modulus as shown in Equation 3.12.

$$
K(t)=C G(t)
$$

where $C$ is an arbitrary constant. If we perform the appropriate Laplace transformations to Equation 3.12 it is apparent that the transformed moduli are related by the same constant $C$ as shown in Equation 3.13.

$$
\frac{\overline{K^{*}}(s)}{\overline{G^{*}}(s)}=\frac{\bar{K}(s)}{\bar{G}(s)}=C
$$

Using this assumption it can be easily shown that this leads to a constant Poisson's ratio as shown in Equation 3.14.

$$
\nu(t)=\nu_{o}=\text { constant }
$$

As the epoxy will be used in its glassy region below the glass transition temperature and that a constant Poisson's ratio is desired for easy integration into the classical lamination theory, the third assumption will be used. With the properties of viscoelastic material described, the structural analysis of composite materials will now be outlined. 


\subsection{Characteristics of Composite Materials}

To determine the structural response of the I-shaped wing spar, the properties of each component must be determined. Since I-beams are composed of two horizontal flanges and a vertical web, a method must be derived to combine their response. Before the overall response of the beam can be determined, the properties of each component must be determined using the Classical Lamination Theory (CLT) [7, 30]. The following will briefly describe CLT and how it is used to determine the extensional, flexural and torsional stiffness of each laminate plate.

\subsubsection{Classical Lamination Theory}

Similar to Euler's bending theory, the classical lamination theory is a method to examine the response of a composite beam or plate by making three distinct assumptions $[7,30]$.

1. Plane sections remain plane and normal to the mid surface of the plate.

2. The material is incompressible in the through thickness direction.

3. The layers are perfectly bonded which allows no slip at the ply interface

To determine the overall properties of a laminated composite plate, structural characteristics of individual plies must be determined [7,31]. The 1, 2, 3 notation is used to represent the local frame of reference as shown in Figure 3.14.

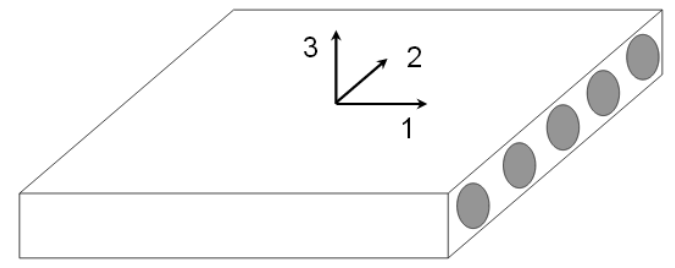

Figure 3.14: Illustration of the local frame of reference for a single ply [7]

To further simplify the analysis, out-of-plane stresses are assumed negligible as their interactions are hard to predict due to other effects such as delamination or debonding [7]. To determine the elastic moduli in the longitudinal and transverse directions, a representative volume element is examined and can be found in Figure 3.15. 


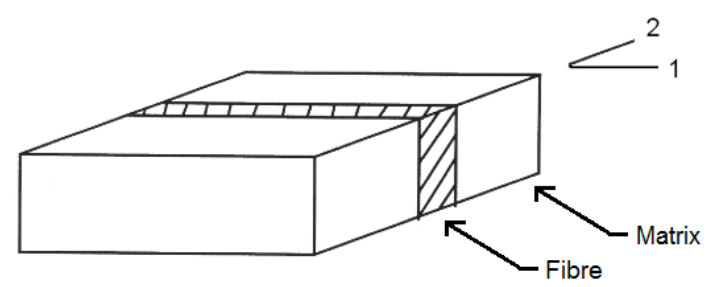

Figure 3.15: Illustration of the representative volume element assumption [7]

Using the rule of mixtures method, the stiffness in the longitudinal and transverse directions, the shear modulus as well as the Poisson's ratio are represented using Equation 3.15 through 3.18.

$$
\begin{gathered}
E_{1}=E_{f} V_{f}+E_{m} V_{m} \\
\frac{1}{E_{2}}=\frac{V_{f}}{E_{f}}+\frac{V_{m}}{E_{m}} \\
\frac{1}{G_{12}}=\frac{V_{f}}{G_{f}}+\frac{V_{m}}{G_{m}} \\
\nu_{12}=\nu_{f} V_{f}+\nu_{m} V_{m}
\end{gathered}
$$

Using these properties, the local compliance and stiffness matrices of each ply are determined respectively using Equations 3.19 and 3.20.

$$
\begin{gathered}
{[S]=\left[\begin{array}{ccc}
1 / E_{1} & -\nu_{12} / E_{1} & 0 \\
& 1 / E_{2} & 0 \\
\text { sym } & 1 / G_{12}
\end{array}\right]} \\
{[Q]=[S]^{-1}}
\end{gathered}
$$

The structural characteristics in the global reference frame can be determined by applying trigonometric transformations to the local properties of each ply. 


\subsubsection{Properties with Respect to Global Frame of Reference}

To transform the local properties to a global x, y, z frame of reference, the transformation matrices for stress $[T]$ and for strain $\left[T^{*}\right]$ are determined for each ply angle using Equations 3.21 and 3.22.

$$
\begin{gathered}
{[T]_{\theta}=\left[\begin{array}{ccc}
c^{2} & s^{2} & 2 s c \\
s^{2} & c^{2} & -2 s c \\
-s c & s c & c^{2}-s^{2}
\end{array}\right]} \\
{\left[T^{*}\right]_{\theta}=\left[\begin{array}{ccc}
c^{2} & s^{2} & s c \\
s^{2} & c^{2} & -s c \\
-2 s c & 2 s c & c^{2}-s^{2}
\end{array}\right]}
\end{gathered}
$$

where $c$ and $s$ represent $\cos \theta$ and $\sin \theta$ respectively. Using the transformation matrices, the stiffness and compliance of each ply are computed in the global x,y,z frame of reference using the following:

$$
\begin{gathered}
{[\bar{S}]_{\theta}=\left[T^{*}\right]_{\theta}^{-1}[S][T]_{\theta}} \\
{[\bar{Q}]_{\theta}=[T]_{\theta}^{-1}[S]\left[T^{*}\right]_{\theta}}
\end{gathered}
$$

Once the properties of each ply are determined in the global frame of reference, the extensional, coupling and flexural stiffness matrices of the entire laminated plate are determined using Equations 3.25 through 3.27 respectively.

$$
\begin{gathered}
{[A]=\sum_{k=1}^{n}[\bar{Q}]_{k} t_{k}} \\
{[B]=\sum_{k=1}^{n}[\bar{Q}]_{k} t_{k} \bar{z}_{k}} \\
{[D]=\sum_{k=1}^{n}[\bar{Q}]_{k}\left(t_{k} \bar{z}_{k}^{2}+\frac{t_{k}^{3}}{12}\right)}
\end{gathered}
$$


where $k, t$ and $\bar{z}$ are the number of plies, ply thickness and the distance from the mid-plane of each ply to the centre of the laminated plate respectively. An illustration of the stacking notation can be found in Figure 3.16.

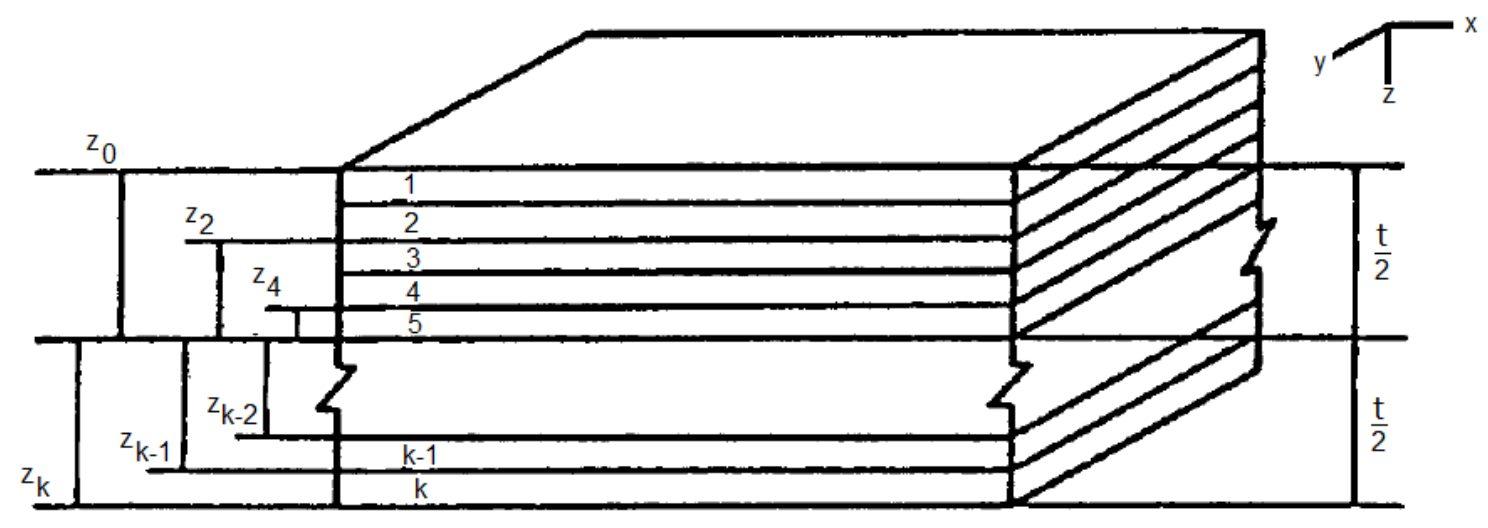

Figure 3.16: Notation used to describe stacking sequence of plies

As the observed stress may vary throughout the plates thickness, it is convenient to introduce the axial and flexural loads in an equivalent form as shown in Equations 3.28 and 3.29 .

$$
\begin{aligned}
& \{N\}=\int_{-\frac{t}{2}}^{\frac{t}{2}}\{\sigma\} d z \\
& \{M\}=\int_{-\frac{t}{2}}^{\frac{t}{2}}\{\sigma\} z d z
\end{aligned}
$$

For axial loads, the composite lamina will respond based on properties of the extensional and coupling matrices as described in Equation 3.30.

$$
\{N\}=[A]\left\{\varepsilon^{o}\right\}+[B]\{\kappa\}
$$

where $\varepsilon^{o}$ and $\kappa$ represent the mid-plane strain and curvature respectively. For bending loads, the composite lamina will respond based on properties of the flexural and coupling matrices as described in Equation 3.31.

$$
\{M\}=[B]\left\{\varepsilon^{o}\right\}+[D]\{\kappa\}
$$


By combining Equations 3.30 to 3.31, the full stiffness matrix can be determined for the laminated plate:

$$
\left\{\begin{array}{l}
N \\
M
\end{array}\right\}=\left[\begin{array}{ll}
{[A]} & {[B]} \\
{[B]} & {[D]}
\end{array}\right]\left\{\begin{array}{l}
\varepsilon^{o} \\
\kappa
\end{array}\right\}
$$

Equation 3.32 is then rearranged to determine the mid-plane strain and curvature of the laminate plate based on the applied equivalent loads $N$ and $M$.

$$
\left.\left\{\begin{array}{l}
\varepsilon^{o} \\
\kappa
\end{array}\right\}=\left[\begin{array}{ll}
{[A]} & {[B]} \\
{[B]} & [D]]^{-1}
\end{array}\right\} \begin{array}{l}
N \\
M
\end{array}\right\}
$$

Using the mid-plane properties above, the strain throughout the laminate is determined using Equation 3.34.

$$
\left\{\begin{array}{c}
\varepsilon_{x} \\
\varepsilon_{y} \\
\gamma_{x y}
\end{array}\right\}=\left\{\begin{array}{c}
\varepsilon_{x}^{o} \\
\varepsilon_{y}^{o} \\
\gamma_{x y}^{o}
\end{array}\right\}+z\left\{\begin{array}{c}
\kappa_{x} \\
\kappa_{y} \\
\kappa_{x y}
\end{array}\right\}
$$

Now that the strain through the thickness is known, the stress within each ply is determined in in the global frame of reference using Equation 3.35.

$$
\left\{\begin{array}{c}
\sigma_{x} \\
\sigma_{y} \\
\tau_{x y}
\end{array}\right\}=[\bar{Q}]_{\theta}\left\{\begin{array}{l}
\varepsilon_{x} \\
\varepsilon_{y} \\
\gamma_{x y}
\end{array}\right\}
$$

To determine the stress experienced by the fibre and matrix directly, the global stresses and strains are transformed into their local frame of reference using the inverse of the transformation matrices determined in Equations 3.21 and 3.22. These transformations are performed using the following equations respectively. 


$$
\begin{aligned}
& \left\{\begin{array}{c}
\sigma_{1} \\
\sigma_{2} \\
\tau_{12}
\end{array}\right\}=[T]_{\theta}^{-1}\left\{\begin{array}{c}
\sigma_{x} \\
\sigma_{y} \\
\tau_{x y}
\end{array}\right\} \\
& \left\{\begin{array}{c}
\varepsilon_{1} \\
\varepsilon_{2} \\
\gamma_{12}
\end{array}\right\}=\left[T^{*}\right]_{\theta}^{-1}\left\{\begin{array}{c}
\varepsilon_{x} \\
\varepsilon_{y} \\
\gamma_{x y}
\end{array}\right\}
\end{aligned}
$$

The method outlined above is the general representation of the Classical Lamination Theory for a simple rectangular cross section with plies orthogonal to the bending direction. To avoid adverse warping from environmental effect such as moisture or temperature, beams are designed using a symmetric layup to eliminate the coupling effects between plies [30]. A convenient way to characterise the engineering constants of a symmetric plate is to examine components of the extensional and flexural compliance matrices.

\subsubsection{Engineering Constants of a Laminated Plate}

For an orthotropic lamina, the stresses and strains can be characterised using the relationship found in Equation 3.38.

$$
\left\{\begin{array}{c}
\varepsilon_{x} \\
\varepsilon_{y} \\
\gamma_{x y}
\end{array}\right\}=\left[\begin{array}{ccc}
1 / E_{1} & -\nu_{12} / E_{1} & C_{1} \\
& 1 / E_{2} & C_{2} \\
s y m & & 1 / G_{12}
\end{array}\right]\left\{\begin{array}{c}
\sigma_{x} \\
\sigma_{y} \\
\sigma_{x y}
\end{array}\right\}
$$

where $C_{1}$ and $C_{2}$ are coupling properties with respect to the shear modulus. Since the stress throughout the laminate varies through its thickness, the average stress in each direction can be characterized using the form:

$$
\{\bar{\sigma}\}=\frac{1}{t} \int_{-\frac{t}{2}}^{\frac{t}{2}}\{\sigma\} d z=\left\{\frac{N}{t}\right\}
$$

where $\bar{\sigma}$ represents the average stress in each direction. Substituting Equation 3.39 into 3.38 yields the relationship between the laminate strain and the average stress 
in a form similar to Equation 3.33.

$$
\left\{\begin{array}{c}
\varepsilon_{x}^{\circ} \\
\varepsilon_{y}^{\circ} \\
\gamma_{x y}^{\circ}
\end{array}\right\}=\frac{1}{t}\left[\begin{array}{ccc}
1 / E_{1} & -\nu_{12} / E_{1} & C_{1} \\
& 1 / E_{2} & C_{2} \\
s y m & & 1 / G_{12}
\end{array}\right]\left\{\begin{array}{c}
N_{x} \\
N_{y} \\
N_{x y}
\end{array}\right\}
$$

For symmetric lamina, it can be assumed that the coupling stiffness is zero and the compliance matrices are the inverse of the stiffness matrices as in Equations 3.41 through 3.43 .

$$
\begin{gathered}
{[b]=[B]=0} \\
{[a]=[A]^{-1}} \\
{[d]=[D]^{-1}}
\end{gathered}
$$

where the lower case notation represents the compliance matrices. Comparing terms, the extensional stiffness in each direction is determined using Equations 3.44 through 3.46 .

$$
\begin{aligned}
& E_{x}^{e x t}=\frac{1}{t a_{11}} \\
& E_{y}^{e x t}=\frac{1}{t a_{22}} \\
& G_{x y}^{e x t}=\frac{1}{t a_{66}}
\end{aligned}
$$

A similar procedure can be used to determine flexural stiffness by correlating flexural compliance to the beam's curvature as in Equation 3.47

$$
\left\{\begin{array}{c}
\kappa_{x} \\
\kappa_{y} \\
\kappa_{x y}
\end{array}\right\}=[d]\left\{\begin{array}{l}
M_{x} \\
M_{y} \\
M_{x y}
\end{array}\right\}
$$


For a rectangular cross section with a unit thickness, one dimensional beam theory states the curvature in the x-direction is represented by Equation 3.48.

$$
\kappa=\frac{M}{E^{\text {flex } I}}=\frac{12 M}{t^{3} E^{\text {flex }}}
$$

where $E^{f l e x}$ is the flexural stiffness in a given direction. Substituting Equation 3.48 into Equation 3.47 and rearranging for the flexural stiffness yields the following.

$$
\begin{aligned}
& E_{x}^{\text {flex }}=\frac{12}{t^{3} d_{11}} \\
& E_{y}^{\text {flex }}=\frac{12}{t^{3} d_{22}} \\
& G_{x y}^{\text {flex }}=\frac{12}{t^{3} d_{66}}
\end{aligned}
$$

To determine the response of beams with complex geometries, CLT method must be elaborated to account for bending in sections where the plies are parallel to the loading direction as well as the effects of the parallel axis theorem. Using these engineering constants, the effective axial, flexural and torsional stiffness of the Ibeam will be derived in the following sections. The method to determine the effective axial stiffness will be outlined first.

\subsubsection{Determination of the Effective Axial Stiffness}

To determine the effective axial stiffness of the composite I-beam, the total axial strain in the span wise direction, $\varepsilon_{x}$, must be determined based on a total axial load $N_{x}^{c}$ acting through the centroid. An illustration of the nomenclature with respect to the composite I-beam is found in Figure 3.17. 

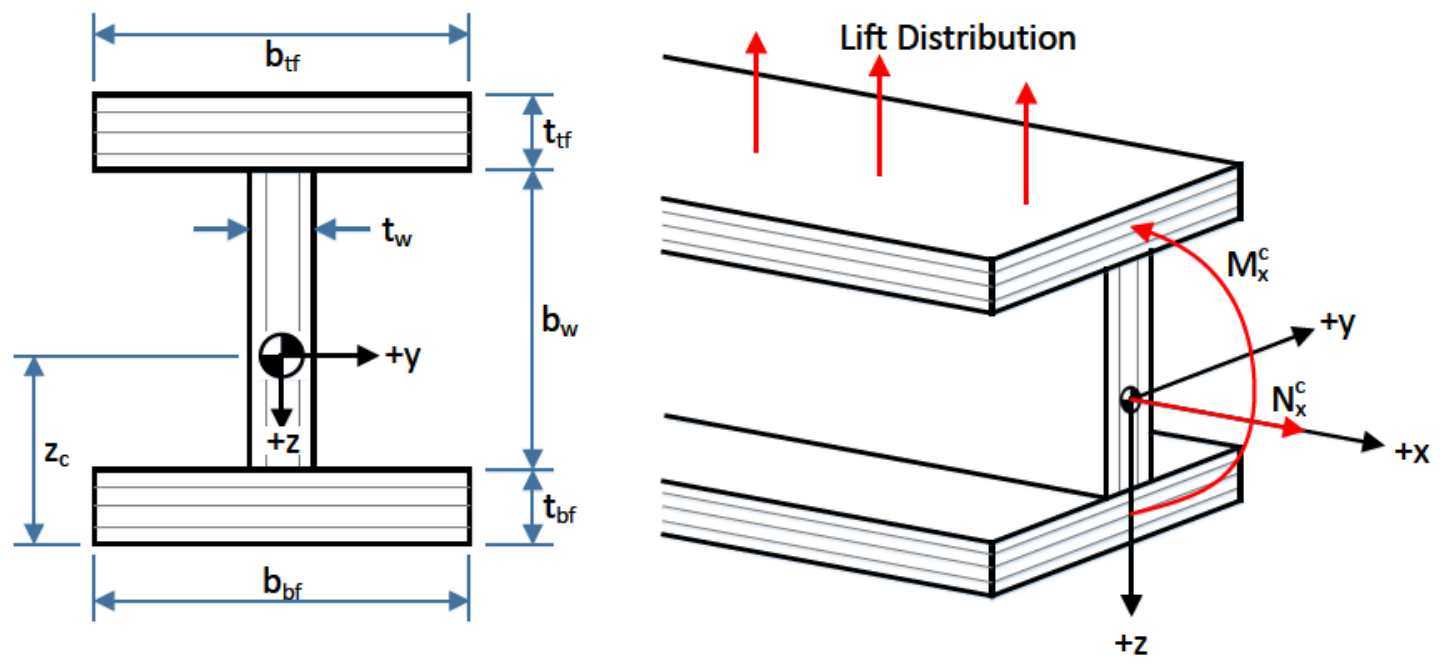

Figure 3.17: Graphical illustration of notation used to describe to the I-beam

Since pure extension is examined, the resultant axial load must be applied through the centroid to ensure a uniform axial strain with no bending. As a result, the centroid of the beam must first be determined based on the properties of the three plates. The location of the centroid is determined by equating the moment in the y-direction to zero as illustrated in Figure 3.18

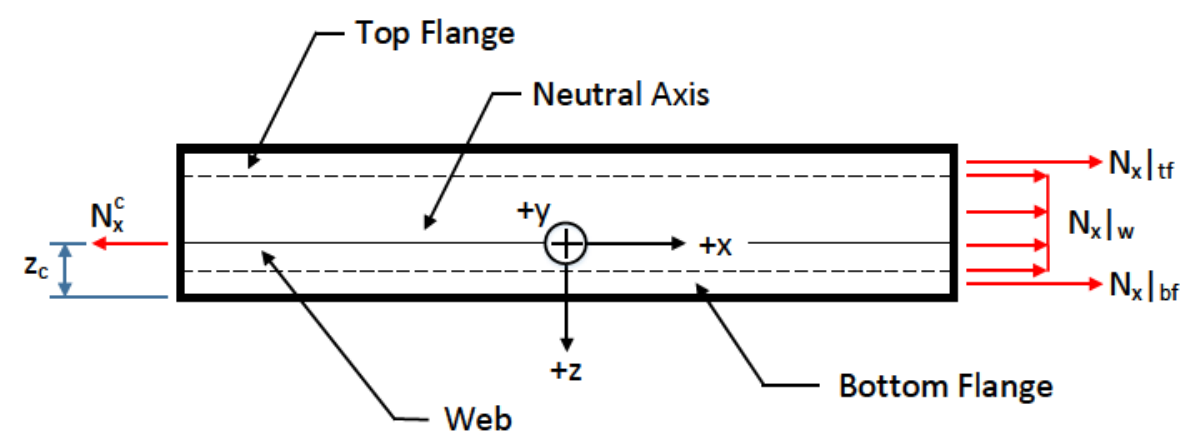

Figure 3.18: Free body diagram illustrating the loads imposed by each component of the I beam in pure extension

$$
\begin{array}{r}
\sum M_{y}=\left[\left.N_{x}\right|_{w} \cdot b_{w}\right]\left(z_{c}-t_{b f}-\frac{b_{w}}{2}\right)+\left[\left.N_{x}\right|_{b f} \cdot b_{b f}\right]\left(z_{c}-\frac{t_{b f}}{2}\right)+ \\
+\left[\left.N_{x}\right|_{t f} \cdot b_{t f}\right]\left(z_{c}-t_{b f}-b_{w}-\frac{t_{t f}}{2}\right)=0
\end{array}
$$


where $z_{c}$ is the location of the centroid in the z-direction. From Equation 3.40, the equivalent axial loads in the longitudinal direction $N_{x}$ for each component can be represented using Equations 3.53 to 3.55.

$$
\begin{aligned}
& \left.N_{x}\right|_{w}=t_{w} E_{x}^{e x t} \varepsilon_{x} \\
& \left.N_{x}\right|_{b f}=t_{b f} E_{x}^{e x t} \varepsilon_{x} \\
& \left.N_{x}\right|_{t f}=t_{t f} E_{x}^{e x t} \varepsilon_{x}
\end{aligned}
$$

where subscripts $w, b f$ and $t f$ represent the web and bottom and top flanges respectively. By substituting Equations 3.53 to 3.55 into Equation 3.52 and rearranging it for the location of the centroid yields the following:

$$
z_{c}=\frac{1}{2}\left[\frac{\left.A_{w} E_{x}^{e x t}\right|_{w}\left(b_{w}+2 t_{b f}\right)+\left.A_{w} E_{x}^{e x t}\right|_{w} t_{b f}+\left.A_{w} E_{x}^{e x t}\right|_{w}\left(2 t_{b f}+2 b_{w}+2 t_{b f}\right)}{A_{w} E_{x}^{e x t}\left|w+A_{b f} E_{x}^{e x t}\right|_{b f}+\left.A_{t f} E_{x}^{e x t}\right|_{t f}}\right]
$$

The second condition that must be met is that the sum of the forces within each component must equal the total force acting through the centroid represented with Equation 3.57 .

$$
N_{x}^{c}=\left.N_{x}\right|_{w} \cdot b_{w}+\left.N_{x}\right|_{b f} \cdot b_{b f}+\left.N_{x}\right|_{t f} \cdot b_{t f}
$$

Using the total applied load, the stress within the beam is defined using elementary mechanics.

$$
\bar{\sigma}_{\text {beam }}=\frac{N_{x}^{c}}{A_{\text {beam }}}=\frac{\left.N_{x}\right|_{w} \cdot b_{w}+\left.N_{x}\right|_{b f} \cdot b_{b f}+\left.N_{x}\right|_{t f} \cdot b_{t f}}{A_{w}+A_{b f}+A_{t f}}
$$

where $\bar{\sigma}_{\text {beam }}$ is the effective extensional stress within the beam. Substituting Equations 3.53 to 3.55 into Equation 3.58 and simplifying yields the following:

$$
\bar{\sigma}_{\text {beam }}=\varepsilon_{x}\left[\frac{\left.A_{w} E_{x}^{e x t}\right|_{w}+\left.A_{b f} E_{x}^{e x t}\right|_{b f}+\left.A_{t f} E_{x}^{e x t}\right|_{t f}}{A_{w}+A_{b f}+A_{t f}}\right]
$$

Comparing this to the general expression for a composite beam found in Equation 3.60, the effective extensional stiffness for the I-beam is defined with Equation 3.61. 


$$
\begin{gathered}
E=\frac{\sum E A}{\sum A} \\
\left.E_{x}^{e x t}\right|_{\text {beam }}=\frac{\left.A_{w} E_{x}^{e x t}\right|_{w}+\left.A_{b f} E_{x}^{e x t}\right|_{b f}+\left.A_{t f} E_{x}^{e x t}\right|_{t f}}{A_{w}+A_{b f}+A_{t f}}
\end{gathered}
$$

Multiplying this by the total area will define the effective axial rigidity of the beam.

$$
\overline{A E}=\left.A_{w} E_{x}^{e x t}\right|_{w}+\left.A_{b f} E_{x}^{e x t}\right|_{b f}+\left.A_{t f} E_{x}^{e x t}\right|_{t f}
$$

With the effective axial stiffness of the I-beam defined, the next step is to define the effective flexural stiffness of the beam.

\subsubsection{Determination of the Effective Flexural Stiffness}

To determine the effective flexural stiffness of the beam, pure bending is examined. As with an isotropic beam, it can be concluded that the neutral axis will pass through the centroid of the I-beam which was defined earlier in Equation 3.56. Figure 3.19 is a free body diagram representing the loads imposed on the centroid by the web and flange sections.

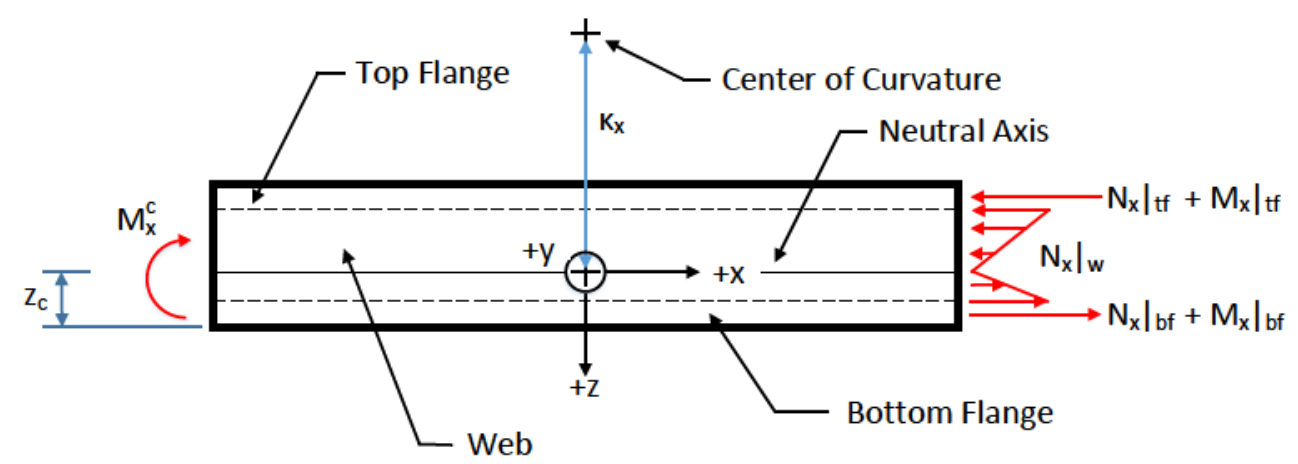

Figure 3.19: Free body diagram illustrating the loads imposed by each component of the I-beam in pure bending

Due to the parallel ply orientation used by the web, the incremental moment it supports is associated with the extensional load $\left.d N_{x}^{\text {total }}\right|_{w}$ and the distance from the neutral axis as with Equation 3.63. 


$$
\left.d M_{x}^{\text {total }}\right|_{w}=\left.\int_{z_{c}-b_{w}-t_{b f}}^{z_{c}-t_{b f}} d N_{x}^{\text {total }}\right|_{w} z=\left.t_{w} E_{x}^{e x t}\right|_{w} \kappa_{x} z^{2} d z
$$

As the thickness, extensional stiffness and curvature are not a function of $z$, the moment supported by the web can be defined by evaluating the integral in Equation 3.63 .

$$
\left.M_{x}^{\text {total }}\right|_{w}=\left(\frac{\left.t_{w} E_{x}^{e x t}\right|_{w} \kappa_{x}}{3}\right)\left[\left(z_{c}-t_{b f}\right)^{3}-\left(z_{c}-b_{w}-t_{b f}\right)^{3}\right]
$$

To determine the total moment held by the flanges, both the force and moment resultant $N_{x}$ and $M_{x}$ must be examined. As illustrated in the Figure 3.19, the equivalent loads $\left.N_{x}\right|_{b f}$ and $\left.N_{x}\right|_{t f}$ are required to account for the parallel offset of the flanges from the neutral axis and the equivalent moment is to account for the beam's curvature.

For simplicity the bottom flange is examined and the same correlation is made for the top flange. As expected with pure bending, the longitudinal axis of the beam will deform in a circular manner with a specific radius of curvature $\kappa_{x}$. Therefore the axial strain will vary linearly in the z-direction as in Equation 3.65.

$$
\varepsilon_{x}=z \kappa_{x}
$$

The mid-plane strain of the flanges is determined by substituting its location along the z-axis into Equation 3.65.

$$
\left.\varepsilon_{x}^{o}\right|_{b f}=\left(z_{c}-\frac{t_{b f}}{2}\right) \kappa_{x}
$$

Based on the classical lamination theory, it was earlier stated that the mid-plane strain of a laminated plate is represented using Equation 3.67.

$$
\left.\varepsilon_{x}^{o}\right|_{b f}=\frac{\left.N_{x}\right|_{b f}}{\left.t_{b f} E_{x}^{e x t}\right|_{b f}}
$$

Equating and rearranging Equations 3.66 and 3.67 for the equivalent load $\left.N_{x}\right|_{b f}$ yields the following:

$$
\left.N_{x}\right|_{b f}=\left(z_{c}-\frac{t_{b f}}{2}\right)\left(\left.t_{b f} E_{x}^{e x t}\right|_{b f}\right) \kappa_{x}
$$

The contribution of the equivalent moment carried by the flanges can be determined by rearranging Equation 3.48. 


$$
\left.M_{x}\right|_{b f}=\frac{\left.t_{b f}^{3} E_{x}^{f l e x}\right|_{b f}}{12} \kappa_{x}
$$

Since both Equations 3.68 and 3.69 are in equivalent form, that is a load per unit length, the total moment supported by the bottom flanges is a summation of Equation 3.68 and 3.69 multiplied by its width as follows:

$$
\left.M_{x}^{t o t a l}\right|_{b f}=\left[\left(z_{c}-\frac{t_{b f}}{2}\right)^{2}\left(\left.b_{b f} t_{b f} E_{x}^{e x t}\right|_{b f}\right)+\frac{\left.b_{b f} t_{b f} E_{x}^{f l e x}\right|_{b f}}{12}\right] \kappa_{x}
$$

where $b_{b f}$ represents the width of the bottom flange. This can be further simplified to Equation 3.71 by recognizing the area $A_{b f}$ and the second moment of area $I_{b f}$

$$
\left.M_{x}^{\text {total }}\right|_{b f}=\left[\left(z_{c}-\frac{t_{b f}}{2}\right)^{2}\left(\left.A_{b f} E_{x}^{e x t}\right|_{b f}\right)+\left.I_{b f} E_{x}^{f l e x}\right|_{b f}\right] \kappa_{x}
$$

Using the method defined above, the moment supported by the top flange can be represented using Equation 3.72.

$$
\left.M_{x}^{t o t a l}\right|_{t f}=\left[\left(z_{c}-t_{b f}-b_{w}-\frac{t_{t f}}{2}\right)^{2}\left(\left.A_{t f} E_{x}^{e x t}\right|_{t f}\right)+\left.I_{t f} E_{x}^{f l e x}\right|_{t f}\right] \kappa_{x}
$$

Taking a moment balance about the x-axis, the total moment supported by the Ibeam, acting through the centroid, is defined as the summation of the total moment supported supported by the web and flanges as depicted in Equation 3.73.

$$
M_{x}^{c}=\left.M_{x}^{t o t a l}\right|_{w}+\left.M_{x}^{t o t a l}\right|_{b f}+\left.M_{x}^{t o t a l}\right|_{t f}
$$

The effective flexural stiffness of a beam $\overline{E I}$ is related to the total bending moment through:

$$
\overline{E I}=\frac{M_{x}^{c}}{\kappa_{x}}
$$

Combining Equations 3.64 and 3.71 through 3.74, the effective flexural rigidity of a composite I-beam is as follows: 


$$
\begin{array}{r}
\overline{E I}=\left.\frac{2}{3} t_{w} E_{x}^{e x t}\right|_{w}\left[\left(z_{b}-t_{b f}\right)^{3}-\left(z_{b}-h-t_{b f}\right)^{3}\right]+\ldots \\
\ldots+\left[\left.\left(z_{b}-\frac{t_{b f}}{2}\right)^{2} A_{b f} E_{x}^{e x t}\right|_{b f}+\left.I_{b f} E_{x}^{f l e x}\right|_{b f}\right]+\ldots \\
\ldots+\left[\left.\left(z_{b}-t_{t f}-h-\frac{t_{t f}}{2}\right)^{2} A_{t f} E_{x}^{e x t}\right|_{t f}+\left.I_{t f} E_{x}^{f l e x}\right|_{t f}\right]
\end{array}
$$

The final step to characterize the response of the I-beam is to determine the effective torsional rigidity of the spar.

\subsubsection{Determination of the Effective Torsional Rigidity}

To determine the effective torsional rigidity of a composite I-beam, pure torsion will be examined. To ensure continuity between the web and flanges the following conditions must be satisfied:

$$
\begin{aligned}
& T_{c}=T_{w}+T_{b f}+T_{t f} \\
& \psi=\psi_{w}=\psi_{b f}=\psi_{t f}
\end{aligned}
$$

where $T$ and $\psi$ are the applied torque and angle of twist respectively. The torque and angle of twist can be related to an equivalent moment and curvature through Equations 3.78 and 3.79 respectively.

$$
\begin{gathered}
M_{x y}=2 b T \\
\kappa_{x y}=\frac{2 \psi}{L}
\end{gathered}
$$

where $b$ is the base width of each laminate plate and $\mathrm{L}$ is length of the beam. An illustration of the beam and the boundary conditions imposed are depicted in Figure 3.20 . 


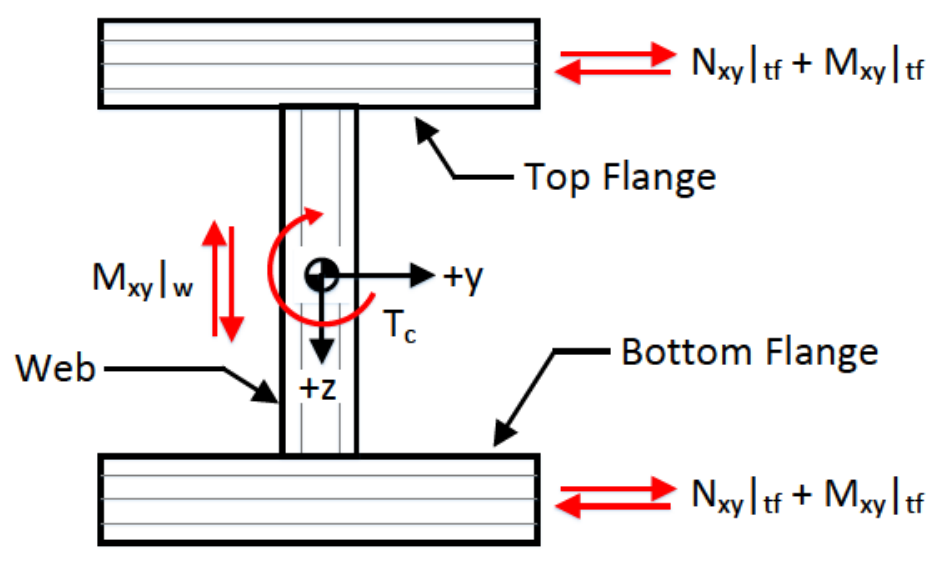

Figure 3.20: Free body diagram illustrating the shear loads imposed on components of the I-beam in pure torsion

To determine the total torque held by the flanges, the analysis will be performed on the bottom flange and then applied to the top flange as done when defining the effective flexural stiffness. To quantify the offsetting distance of the flange from the centroid, an effective axial shear load $N_{x y}$ will be applied. To determine this load, the mid-plane shear strain will be equated in a similar manner to Equations 3.66 through 3.68 .

$$
\begin{gathered}
\left.\gamma_{x y}^{o}\right|_{b f}=\left(z_{c}-\frac{t_{b f}}{2}\right) \frac{\psi}{L} \\
\left.\gamma_{x y}^{o}\right|_{b f}=\frac{\left.N_{x}\right|_{b f}}{\left.t_{b f} G_{x y}^{e x t}\right|_{b f}} \\
\left.N_{x y}\right|_{b f}=\left(z_{c}-\frac{t_{b f}}{2}\right)\left(\left.t_{b f} G_{x y}^{e x t}\right|_{b f}\right) \frac{\kappa_{x y}}{2}
\end{gathered}
$$

The contribution of the equivalent moment carried by the flanges can be determined by rearranging Equation 3.48 for the xy-plane.

$$
\left.M_{x y}\right|_{b f}=\frac{\left.t_{b f}^{3} G_{x y}^{f l e x}\right|_{b f}}{12} \kappa_{x y}
$$

By taking the sum of Equations 3.82 and 3.83 and rearranging for torsion, the total torque applied to the bottom flange can be determined using Equations 3.84. 


$$
\left.T^{\text {total }}\right|_{b f}=\left[\left(z_{c}-\frac{t_{b f}}{2}\right)^{2}\left(\left.A_{b f} G_{x y}^{e x t}\right|_{b f}\right)+\left.4 I_{b f} G_{x y}^{f l e x}\right|_{b f}\right] \kappa_{x y}
$$

Using the method defined above, the moment supported by the top flange can be represented using Equation 3.85.

$$
\left.T^{t o t a l}\right|_{t f}=\left[\left(z_{c}-t_{b f}-b_{w}-\frac{t_{t f}}{2}\right)^{2}\left(\left.A_{t f} E_{x}^{e x t}\right|_{t f}\right)+\left.4 I_{t f} G_{x y}^{f l e x}\right|_{t f}\right] \kappa_{x y}
$$

To determine the torque applied to the web, the form of Equation 3.86 is used; however adapted for the web characteristics.

$$
\left.M_{x y}\right|_{w}=\frac{\left.t_{w}^{3} G_{x y}^{f l e x}\right|_{w}}{12} \kappa_{x y}
$$

The torque held by the web is then determined by substituting Equation 3.78 into Equation 3.86 and rearranging for total torque.

$$
\left.T^{t o t a l}\right|_{w}=\left[\left.2 I_{w} G_{x y}^{f l e x}\right|_{w}\right] \kappa_{x y}
$$

Using Equations 3.84, 3.85, 3.87 and a general form that represents torsion in Equation 3.88, the effective torsional rigidity is determined using Equation 3.89.

$$
\begin{gathered}
\overline{G J}=\frac{2 T_{c}}{\kappa_{x y}} \\
\overline{G J}=2\left[\left.I_{w} G_{x y}^{\text {flex }}\right|_{w}+\left(z_{c}-\frac{t_{b f}}{2}\right)^{2}\left(\left.A_{b f} G_{x y}^{e x t}\right|_{b f}\right)+\left.4 I_{b f} G_{x y}^{\text {flex }}\right|_{b f}+\ldots\right. \\
\left.\ldots+\left(z_{c}-t_{b f}-b_{w}-\frac{t_{t f}}{2}\right)^{2}\left(\left.A_{t f} E_{x}^{e x t}\right|_{t f}\right)+\left.4 I_{t f} G_{x y}^{\text {flex }}\right|_{t f}\right]
\end{gathered}
$$

Using the axial, flexural and torsional rigidities of the composite I-beam, the deflections of the spar can be determined in the same manner as an isotropic material with a defined loading profile. 


\subsection{Wing Deflection Given Arbitrary Loading Profile}

During flight, the loading profile observed by the wing spar will vary considerably depending on the type of manoeuvre. As a result, direct integration of the loading profile is used to determine the applied moments and resulting deflections observed by the wing spar. The moment distribution can be determined by taking the second integral of the loading profile as shown in Equation 3.90

$$
M(x)=\int_{0}^{L} \int_{0}^{L} f(x) d x d x
$$

where $M(x)$ and $f(x)$ are the moment distribution and loading profile and $L$ represents the span of the wing spar. To determine the deflection of the spar, the relationship between curvature and bending moment is examined below:

$$
\frac{1}{\kappa}=\frac{M(x)}{E I}
$$

Noting that the curvature of the beam ${ }^{1} / \kappa_{\kappa}$ can be related to the vertical deflection $v$ as follows through Equation 3.92.

$$
\frac{1}{\kappa}=\frac{d^{2} v(x)}{d x^{2}}
$$

Substituting Equation 3.92 into 3.91 yields an equation that can be directly integrated to determine the slope and deflection of the wing spar as shown in Equations 3.93 and 3.94 respectively.

$$
\begin{gathered}
\theta(x)=\frac{d v(x)}{d x}=\int \frac{-M(x)}{E I} d x+C_{1} \\
v(x)=\frac{d \theta(x)}{d x}=\int\left(\int \frac{-M(x)}{E I} d x\right)+C_{1} x+C_{2}
\end{gathered}
$$

Based on the boundary conditions for a cantilevered beam, the constant of integration $C_{1}$ reduces to zero and $C_{2}$ will vary depending on the loading profile. Since a composite beam is employed for the wing spar, the effective flexural stiffness $\overline{E I}$ is used instead of the product of the second moment of area $I$ and the Young's modulus $E$. With a method to determine the stresses and deflections within the spar, suitable failure criterion can be imposed to determine if or when the composite spar will fail. 


\subsection{Failure Criterion}

There are various methods to determine failure within a composite structure, each with their specific advantages. To determine failure within the Katana wing spar two theories are examined. The first method is the more advanced Tsai-Hill theory and the second is the more conservative maximum strain theory. Since the wing spar experiences a compressive load on the upper flange, stability analysis is performed to ensure that local buckling of the compressive flange does not occur.

\subsubsection{Tsai-Hill Failure Theory}

The Tsai-Hill failure theory is an interactive theory based on the Von Mises distortional energy criteria for an isotropic material but applied to an anisotropic system. This theory only takes into account the distortional strain energy in the system instead of the total strain energy as would be seen by the Tsai-Wu Theory [7,30]. In addition, this criteria does not distinguish between the tensile and compressive strengths of the lamina. Compressive strengths of the fibre are not provided by the manufacturer and the testing of this is not an option with the current equipment; however this is used to our advantage as the compressive strengths of composite materials tend to be higher than there tensile strength. Based on this criteria, failure is considered if the following expression is exceeded $[7,30]$.

$$
\left[\frac{\sigma_{1}}{\left.\sigma_{1}^{T}\right|_{\text {ult }}}\right]^{2}-\left[\frac{\sigma_{1} \sigma_{2}}{\left(\left.\sigma_{1}^{T}\right|_{\text {ult }}\right)^{2}}\right]+\left[\frac{\sigma_{2}}{\left.\sigma_{2}^{T}\right|_{\text {ult }}}\right]^{2}+\left[\frac{\tau_{12}}{\left.\tau_{12}\right|_{\text {ult }}}\right]^{2}<1
$$

To determine the ultimate tensile strength, the rule of mixture is applied as shown in Equations 3.96 and 3.97 [7].

$$
\begin{gathered}
\left.\sigma_{1}^{T}\right|_{u l t}=\left.\sigma_{f}^{T}\right|_{u l t} V_{f}+\left.\sigma_{m}^{T}\right|_{u l t} V_{m} \\
\left.\sigma_{2}^{T}\right|_{u l t}=\frac{V_{f}}{\left.\sigma_{f}^{T}\right|_{u l t}}+\frac{V_{m}}{\left.\sigma_{m}^{T}\right|_{u l t}}
\end{gathered}
$$

The ultimate shear strength of a composite lamina is usually dictated by the shear strength of the matrix as the ply interface is usually the weakest link. As a result, a semi-empirical formulation has been adopted by the industry and is represented in Equation 3.98 [32]. 


$$
\left.\tau_{12}\right|_{u l t}=\frac{\left.\tau_{m}\right|_{u l t}}{\left(1+V_{f}\right)^{2}}
$$

where $V_{f}$ is the fibre volume fraction of the composite material. With safety being a concern in the industry, the more conservative maximum stress or strain criterion are typically used instead of the Tsai-Hill and Tsai-Wu theories. For example, as the viscoelastic matrix begins to creep, the fibres may experience an elongation which is beyond its breaking point. As a result the second failure theory to be examined is the more conservative maximum strain theory.

\subsubsection{Maximum Strain Theory}

The maximum strain failure theory assumes that failure has occurred if the local strain experienced by a ply has exceeded a given amount. This tends to be a more conservative method to determine failure as it does not account for multi-dimensional interactions [7]. As the matrix modulus decreases, the strain observed by each ply will increase and are compared to the fibres maximum elongation listed in manufacturer's specifications. As carbon and glass fibres tend to exhibit brittle failure, failure will be considered if the strain within a ply exceeds $50 \%$ of specified maximum, which represents a safety factor of 2 . If the composite structure has passed these criterion, stability analysis is performed to ensure local buckling of the compressive flange does not occur.

\subsubsection{Local buckling of the Flange or Web}

It has been demonstrated that local buckling will occur in the compressive flange and web if the forces exceeds the critical buckling load. Typically for I-profiles, the compressive flange will buckle first which leads to the immediate buckling of the now unsupported web as depicted in Figures 3.21 and 3.22 [31].

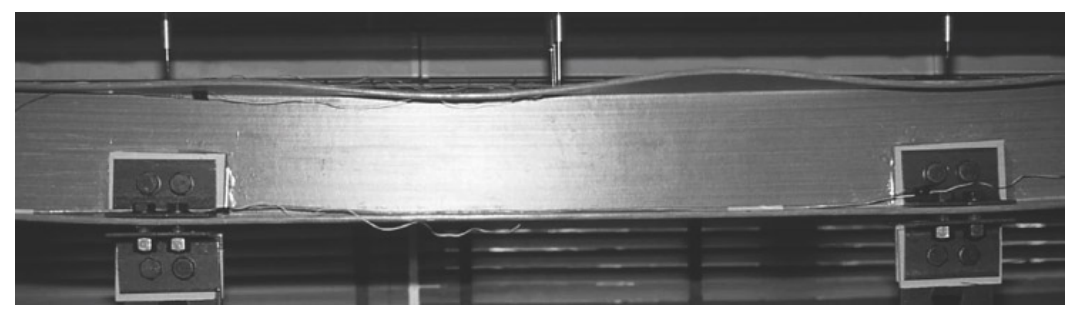

Figure 3.21: Initial local buckling of the compressive flange [31] 


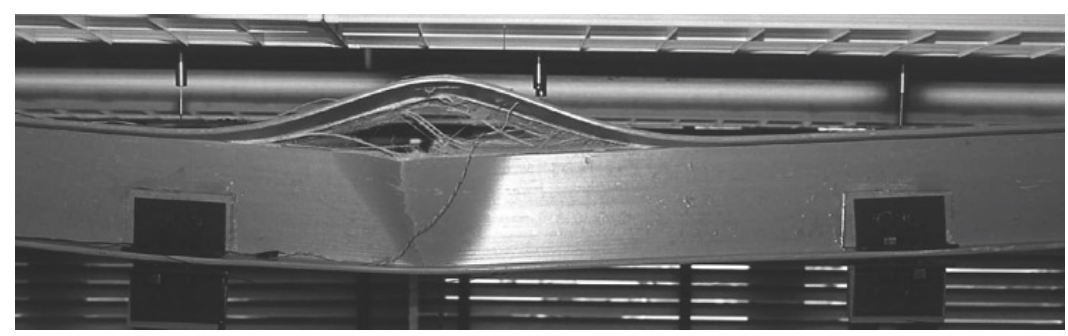

Figure 3.22: Ultimate failure of the web and beam following the buckling of the flange [31]

The critical buckling load of the web and flange are a function of boundary conditions on the longitudinal edges of the walls. The boundary conditions imposed on the flange and web are illustrated in Figure 3.23. It is important to note that only half of the flange is being observed, with the simply supported restraining point located at intersection of the web and flange.

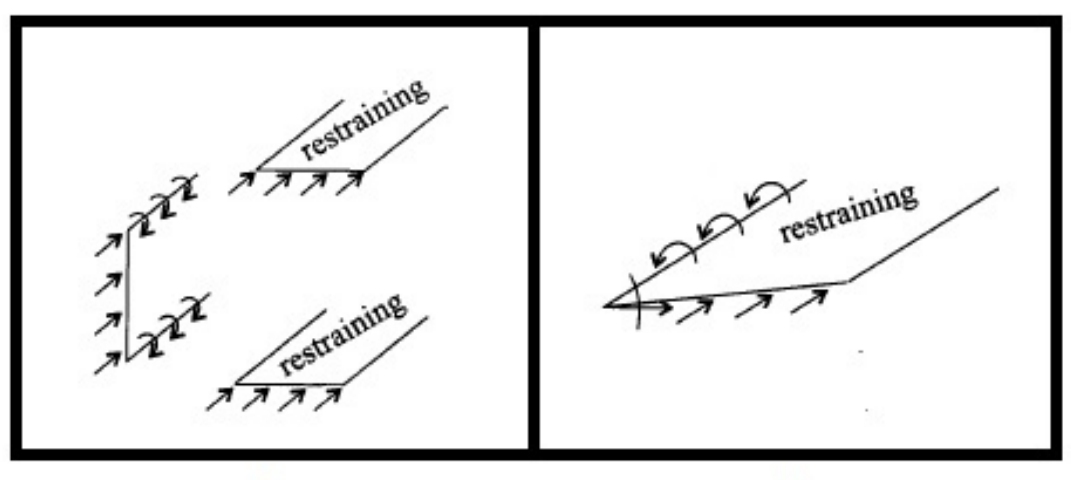

A

B

Figure 3.23: Illustration of the boundary conditions imposed on the web (A) and the half flange (B) [33]

In practice, a flange is characterized as a plate rotationally restrained on one end and free on the other. Currently there is no closed form solution for these boundary conditions however an approximate solution has been proposed [31,33]. To analyse local buckling of a composite I-beam, two simply supported boundary conditions are used to model the web and flange and are found in Equations 3.99 and 3.100 respectively.

$$
\left(N_{s s}^{s s}\right)_{w e b}=\frac{\pi^{2}}{\left(b_{w}\right)^{2}}\left(13.9 \sqrt{D_{11} D_{22}+11.1 D_{12}+22.2 D_{66}}\right)
$$




$$
\left(N_{\text {free }}^{\text {ss }}\right)_{\text {flange }}=\frac{12 D_{66}}{\left(0.5 b_{f}\right)^{2}}
$$

where $b_{w}, b_{f}$ and $D_{1, j}$ are the base widths of the web and the compressive flange and components of the flexural stiffness matrix respectively respectively. It has been determined that the flange will buckle first if the following expression is true:

$$
\left(N_{\text {free }}^{s s}\right)_{\text {flange }}\left(a_{11}\right)_{\text {flange }}<\left(N_{s s}^{s s}\right)_{\text {web }}\left(a_{11}\right)_{\text {web }}
$$

where $a_{11}$ is the inverse of the extensional stiffness matrix. To determine the critical buckling load of the compressive flange, a restraining coefficient is required to model the web flange interaction. The coefficient of restraint is determined using Equation 3.102 .

$$
\zeta_{\text {flange }}=\frac{D_{22}}{k_{\text {flange }}\left(0.5 b_{f}\right)}
$$

where $k_{\text {flange }}$ is an effective spring constant represented by:

$$
k_{\text {flange }}=\frac{2\left(D_{22}\right)_{w e b}}{b_{w}}\left(1-\frac{\left(N_{\text {free }}^{s s}\right)_{\text {flange }}\left(a_{11}\right)_{\text {flange }}}{\left(N_{s s}^{s s}\right)_{w e b}\left(a_{11}\right)_{w e b}}\right)
$$

Using the properties of the compressive flange and the coefficient of restraint, the critical buckling load of the flange is determined through Equation 3.104.

$$
N_{c r}^{\text {flange }}=\frac{1}{\left(0.5 b_{f}\right)^{2}}\left(7 \sqrt{\frac{D_{11} D_{22}}{1+4.12 \zeta_{\text {flange }}}}+12 D_{66}\right)
$$

This formulation underestimates the critical buckling load by as much as $14 \%$ however this provides an inherent safety factor. In literature, there is a more complex formulation which can predict the buckling load to as much as two percent of experimental results [33]. For bending about the longitudinal axis, the critical moment is determined using Equation 3.105.

$$
M_{c r}^{\text {flange }}=N_{c r}^{\text {flange }} b_{f} S_{x}
$$

where $S_{x}$ is the section modulus for an I-beam. For typical geometries and fibre layups, experience has shown that the local buckling of the flange will occur before it is experienced by the web; however if the following expression is satisfied the web 
will buckle before the flange [31]:

$$
\left(N_{\text {free }}^{\text {ss }}\right)_{\text {flange }}\left(a_{11}\right)_{\text {flange }}>\left(N_{s s}^{s s}\right)_{\text {web }}\left(a_{11}\right)_{\text {web }}
$$

There is no exact solution to predict the critical buckling load of the web; however Equation 3.99 is a conservative estimate as the flanges do not provide adequate rotation support [31]. Equation 3.107 is the critical moment required for the buckling of the web section.

$$
M_{c r}^{w e b}=N_{c r}^{w e b} b_{w} \frac{2 I_{x}}{d-2 t_{f}}
$$

This chapter has outlined the theory required to determine the service life of the composite wing spar based on viscoelastic phenomena. It began with a method to analyse the viscoelastic properties of the epoxy matrix through the use time temperature superposition principal. This principal allows the user to create a master curve assuming the Arrhenius form found in Equation 3.8. Once the curve has been created, a Prony series is fit to the data using nonlinear regression techniques and will take the form of Equation 3.5. The mechanical response of the Katana wing spar is then determined using the Classical Lamination Theory with modification for an Ishaped profile. This includes techniques to analyse laminate plates with plies parallel to the direction of bending and to account for the parallel offset of the flanges from the neutral axis. Failure of the wing spar is assumed when the stress or strain in an individual ply exceeds the maximum allowable determined through the Tsai-Hill or maximum strain failure criterion. Finally, stability analysis was outlined to determine if the compressive flange will resist local buckling. The following chapter will outline the experimental setup and procedure used to obtain the creep and relaxation data. 


\section{Chapter 4}

\section{Experimental Setup}

This chapter will outline the experimental setup and procedures used to acquire the viscoelastic characteristics of the MGS L285/H287 epoxy system. It will begin by describing the experimental equipment and the LabVIEW acquisition software which is followed by a test plan and a description of the test specimen. The chapter will finish with detailed instructions on specimen manufacturing as well as on the safe operation of the equipment.

\subsection{Testing Platform}

As described in Section 2.3, various techniques are available to measure the response of viscoelastic materials; however the tension test was selected as it directly measures the time dependant properties of creep and relaxation in a cost effective manner. To determine these characteristics, a testing platform was required to meet the following two criterion.

1. Able to apply uni-axial tension to the specimen in a manner that the load and displacement are measured.

2. Able to sustain and measure temperatures below $100{ }^{\circ} \mathrm{C}$ in a controlled manner.

As a suitable testing platform was not available, it was decided that a decommissioned undergraduate equipment used to measure creep in lead could be retrofitted for use with polymers. An illustration of the former undergraduate equipment is found in Figure 4.1. 


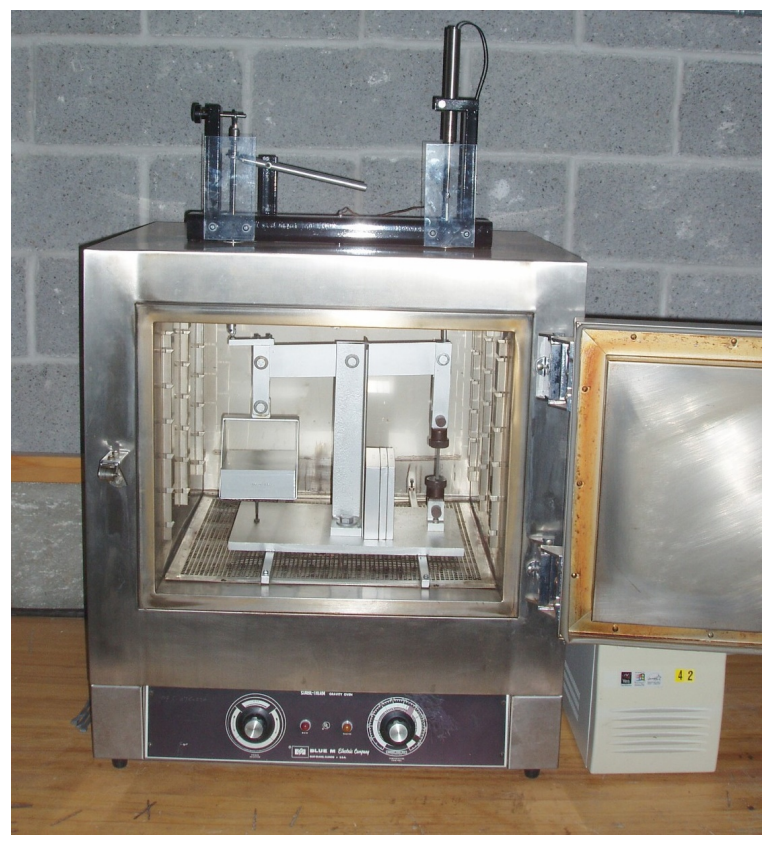

Figure 4.1: Existing undergraduate equipment used to demonstrate creep with lead.

Based on test requirements, the new testing platform employs the low temperature oven to control the temperature and a linear actuator to apply the load as depicted in Figure 4.2. Although it is ideal to perform creep tests with weights to ensure a consistent load [34], it was decided that a linear actuator in conjunction with a calibrated load cell should be used to allow for a broader range of tests including the required relaxation test. 


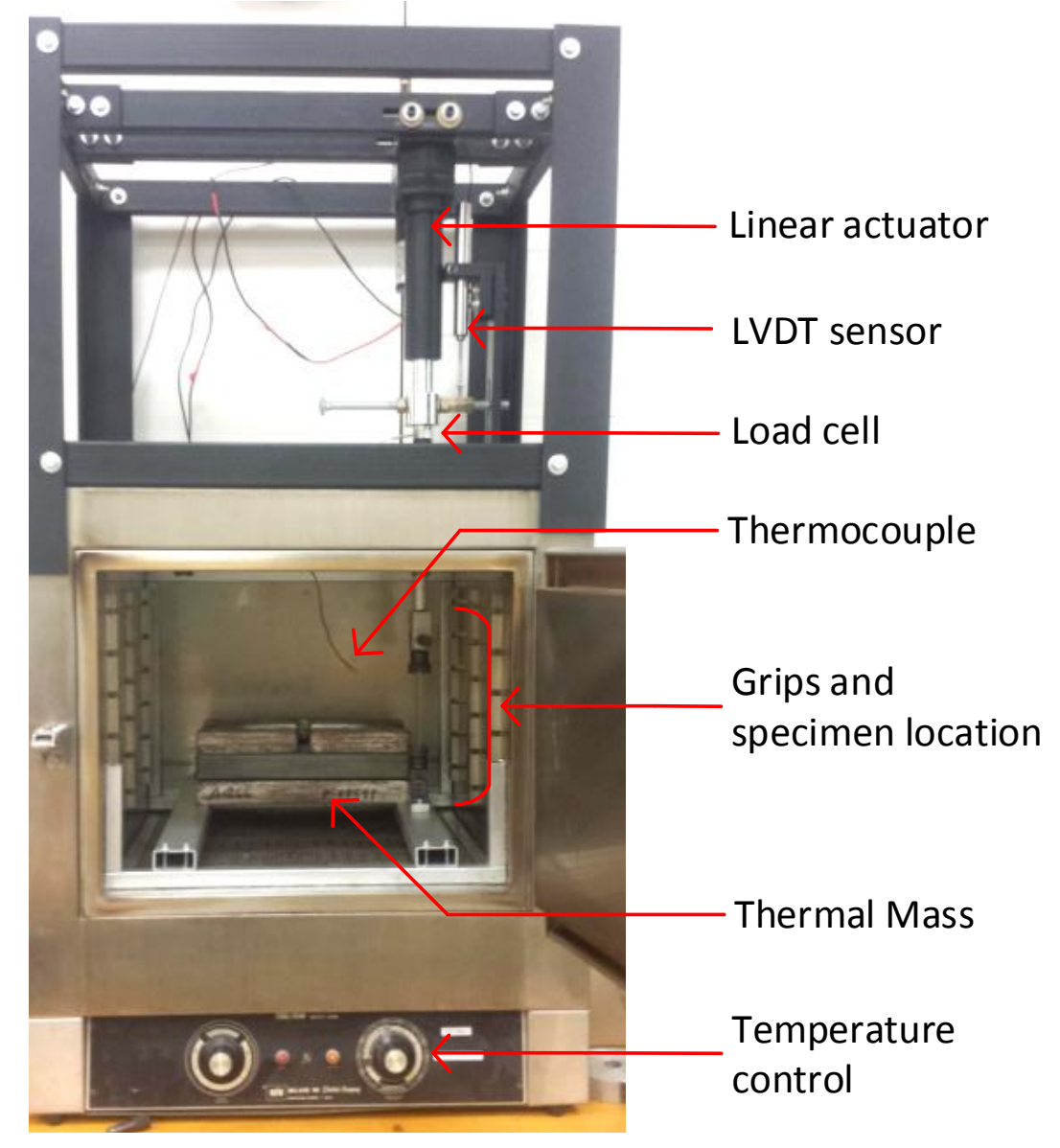

Figure 4.2: Former set up for the creep and relaxation tests

Employing parts from the decommissioned setup, the data is acquired using National Instruments LabVIEW and the USB-6008 data acquisition device. This allows for monitoring of the temperature, applied load and resulting displacement. The following section will explain the LabVIEW interface and how it will be used to perform the desired tests.

\subsection{National Instruments LabVIEW}

National Instruments LabVIEW is a program that allows for easy data acquisition due to the user friendly interface. Instead of using text based code, it allows the user to program using a flow diagram and function blocks. Figures 4.3 and 4.4 are illustrations of the data acquisition unit and an example of the LabView interface. 


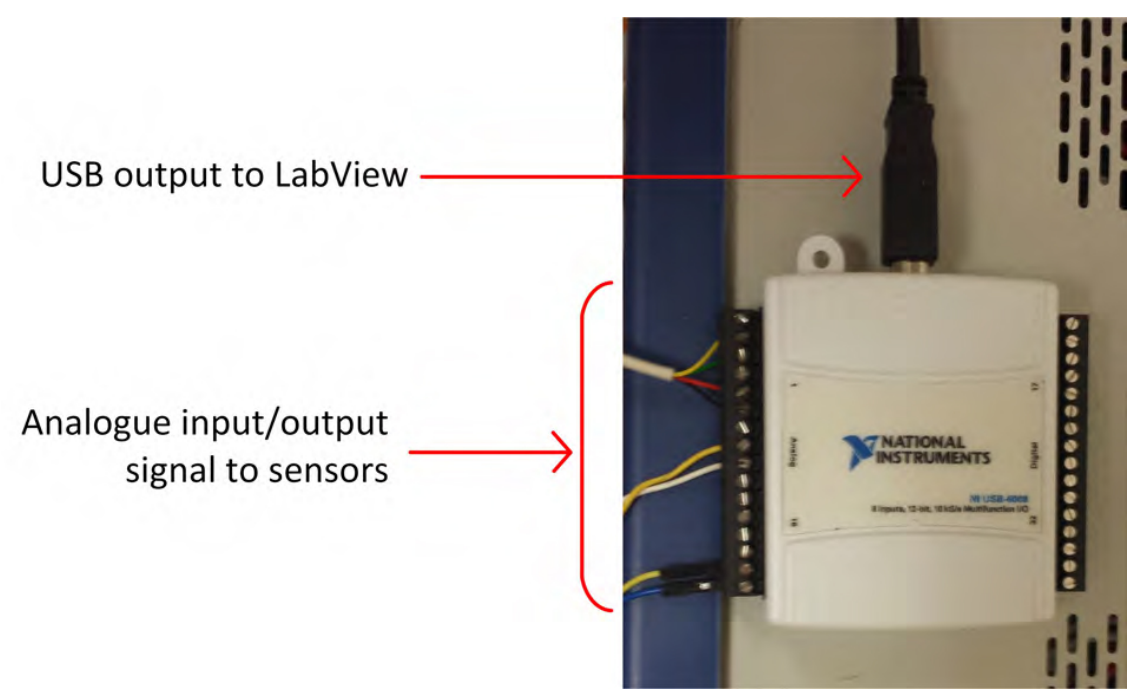

Figure 4.3: National Instrument USB-6008 Data acquisition unit

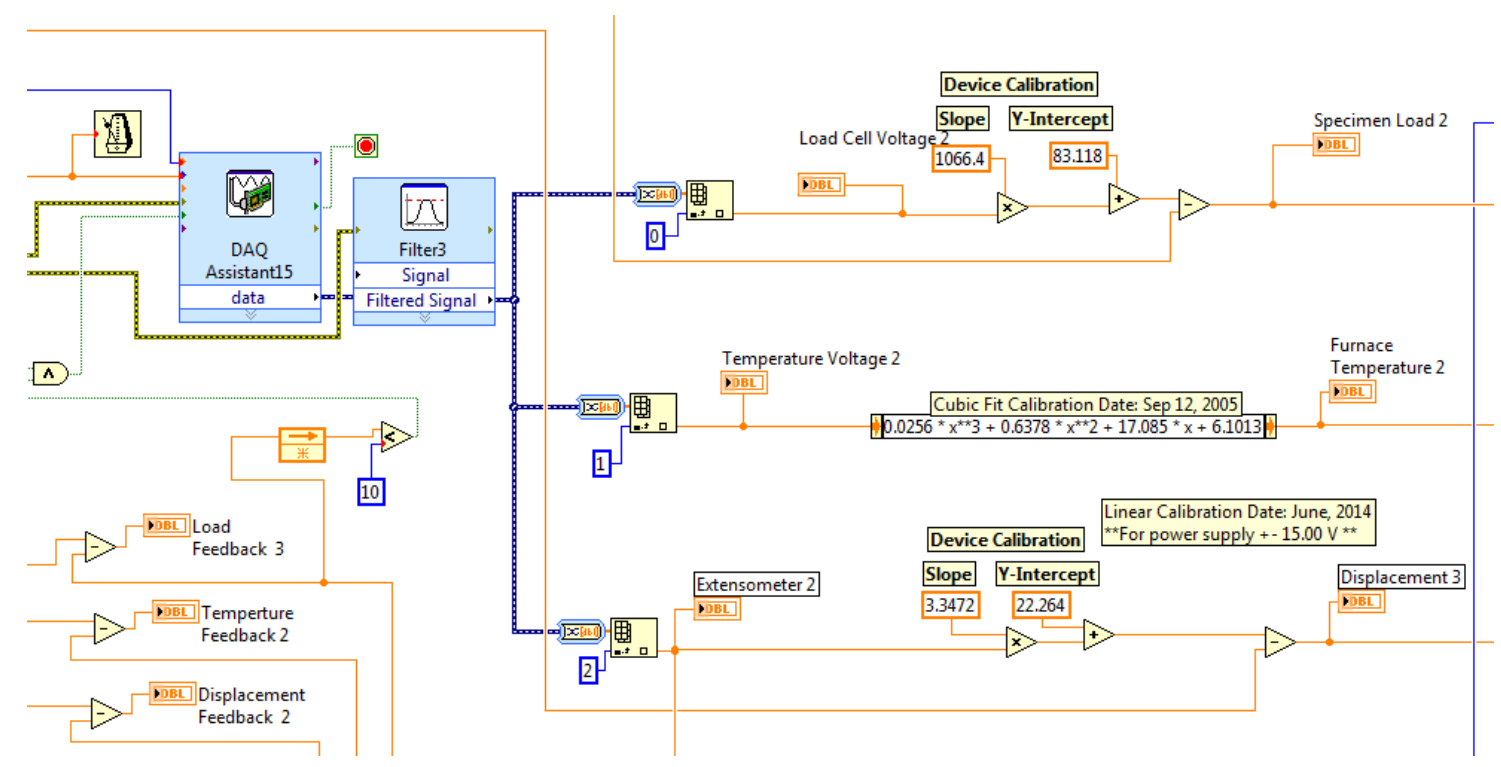

Figure 4.4: Example of LabVIEW programing interface

To obtain the necessary data two similar programs were created, one to obtain creep data and the second to collect relaxation data. Each program begins by zeroing the sensors and asking for specimen dimensions and test parameters. Both the creep and relaxation programs can be broken down into three phases. The first phase is focused on the initial loading of the specimen to determine initial modulus. This is typically conducted at low strain rates to ensure inertial forces are kept to a minimum [18]. The 
second phase is the main test and its focus is to determine the creep and relaxation properties of the material described in Section 3.2. The final phase is the unloading of the specimen and it is used to determine the unloaded length and the recovery characteristics of the material. Based on this information, an illustration of a typical loading cycle can be found in Figure 4.5.

\section{General Loading Cycles for the Creep and Relaxation Tests}

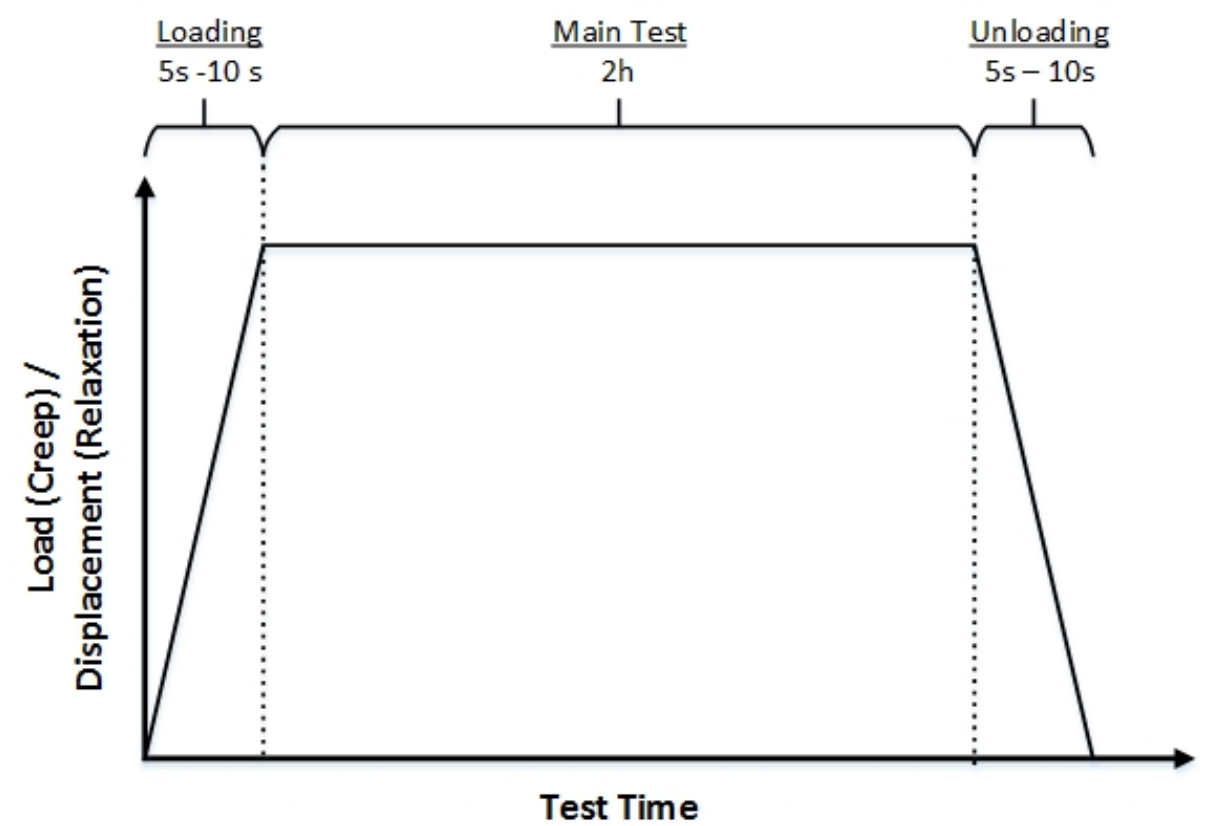

Figure 4.5: Approximate loading cycle for the creep and relaxation tests

As a result of a limited memory capacity, it was decided that the duration of the main test would be two hours; however, at higher temperature the test times were reduced as the specimen would fully relax before the two hours have elapsed. The loading and unloading times are approximations based on the actuating speed for which the control system is described in the following sections.

\subsection{Sensors and Actuation Control}

To collect the data a linear variable differential transformer (LVDT), load cell and K-type thermocouple were required to collect the displacement, load and temperature 


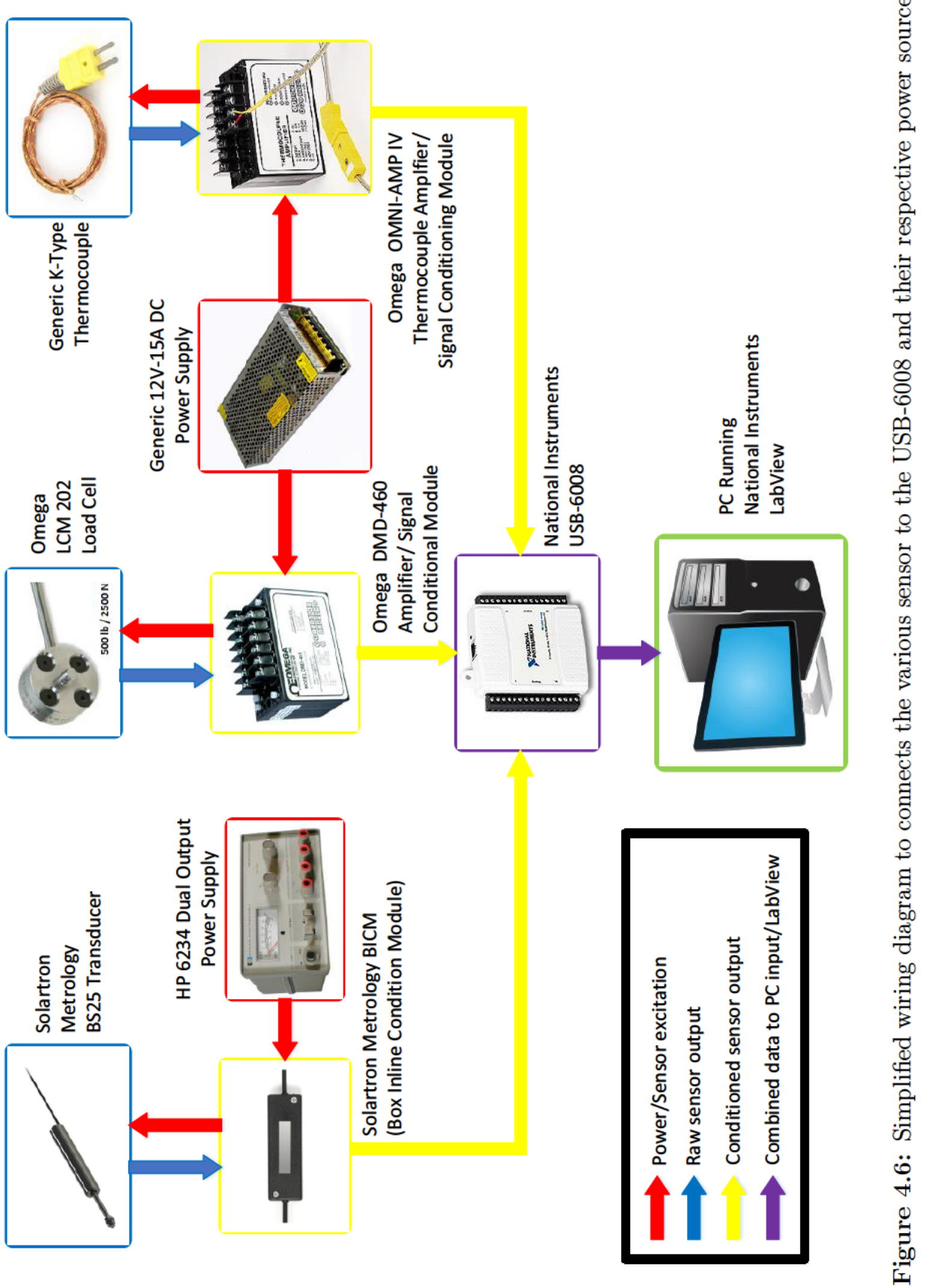


respectively. Since these sensors are based on a voltage differential, signal conditioning units are required between each sensor and the USB-6008 to provide the proper excitation and to help reduce output noise. Figure 4.6 is a simplified wiring diagram from each sensor to the USB-6008 data acquisition unit.

These voltages are converted to their respective units in LabVIEW through calibration coefficients determined using a linear correlation through a series of known data points. The load cell was calibrated using a $25 \mathrm{kN}$ MTS machine and the LVDT was calibrated using a micrometre with a $2 "(50.8 \mathrm{~mm})$ stroke. The calibration coefficients used to convert the raw instrument voltages to their respective units can be found in Table 4.1.

Table 4.1: Calibration coefficients to convert sensor voltage to their respective units

\begin{tabular}{|l|l|}
\hline Sensor Type & Calibration Coefficients \\
\hline Load Cell & Load $=1066.4 V+83$ \\
\hline LVDT & Distance $=2 \mathrm{~V}+2$ \\
\hline Thermocouple & Temperature $=0.0256 V^{3}+0.6378 V^{2}+17.085 V+6.1013$ \\
\hline
\end{tabular}

Note: $V=$ Sensor Voltage

To properly control the electric actuator, an H-Bridge was installed between the power and signal source. This device consists of four solid state MOSFET switching relays which allows for directional control and pulse width modulation to control the actuating speed. Figures 4.7 and 4.8 are illustrations of a basic diagram of an H-bridge circuit and a simplified wiring diagram to control the linear actuator respectively. 


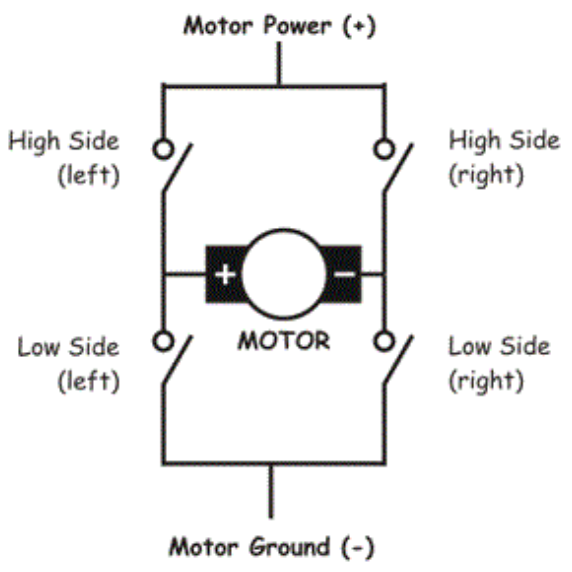

Figure 4.7: Circuit diagram of basic H-Bridge motor controller [35]

For an adequate set of data, the creep and relaxation tests are performed at various temperatures, loads or strains. After the data is collected, it is exported into spreadsheets and analysed in MathWorks MATLAB. The results of this analysis can be found in Chapter 5 .

\subsection{Specimen Manufacturing and Testing Proce- dure}

ASTM International is an engineering society that provides standards for the testing of materials. To perform creep tests on unreinforced plastics, ASTM Standard D638 "Standard Test Method for Tensile Properties of Plastics" recommends the use of a specimen with a rectangular cross section with the dimensions shown in Figure $4.9[36]$. 


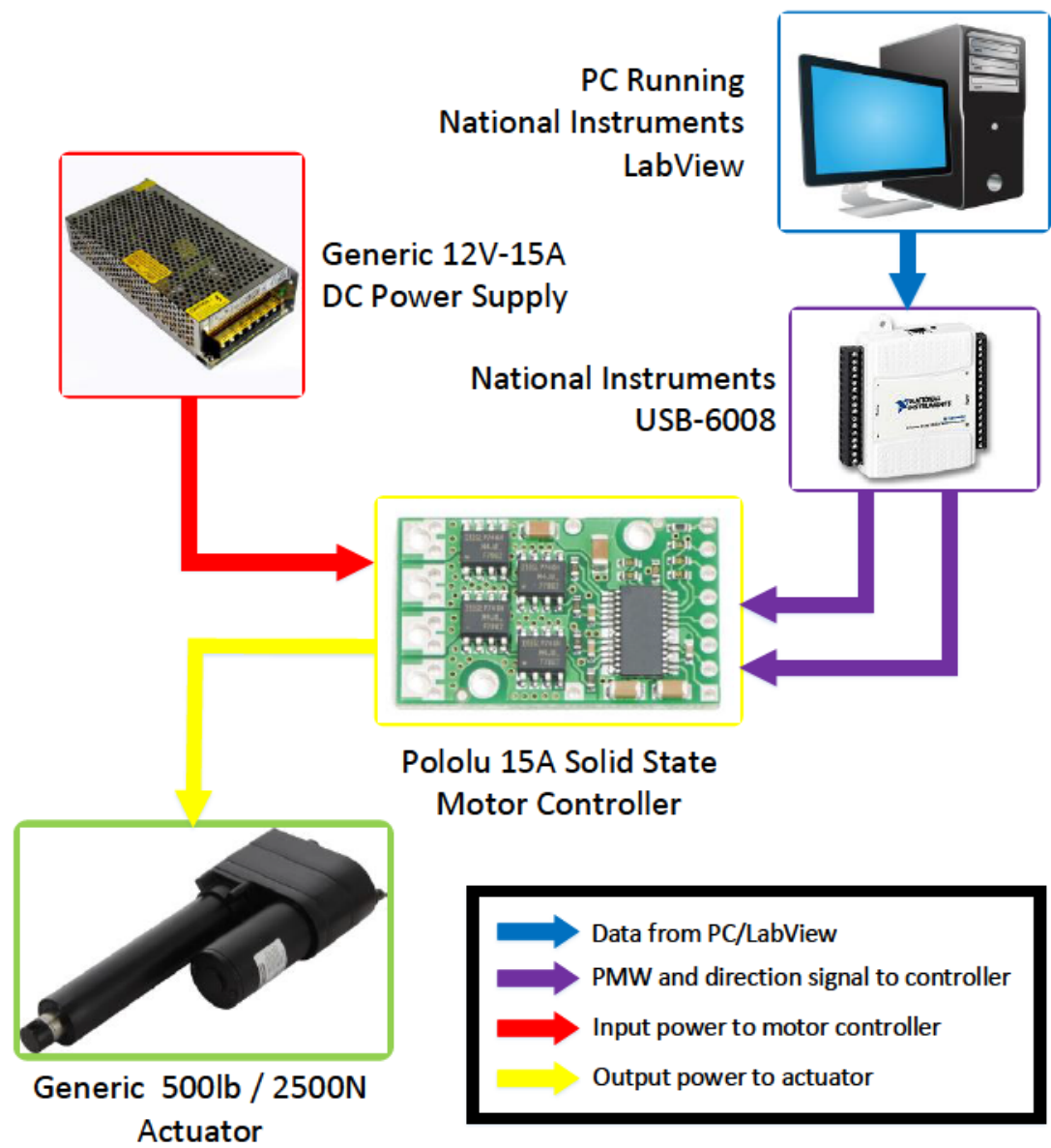

Figure 4.8: Simplified wiring diagram to control the 2500N linear actuator 


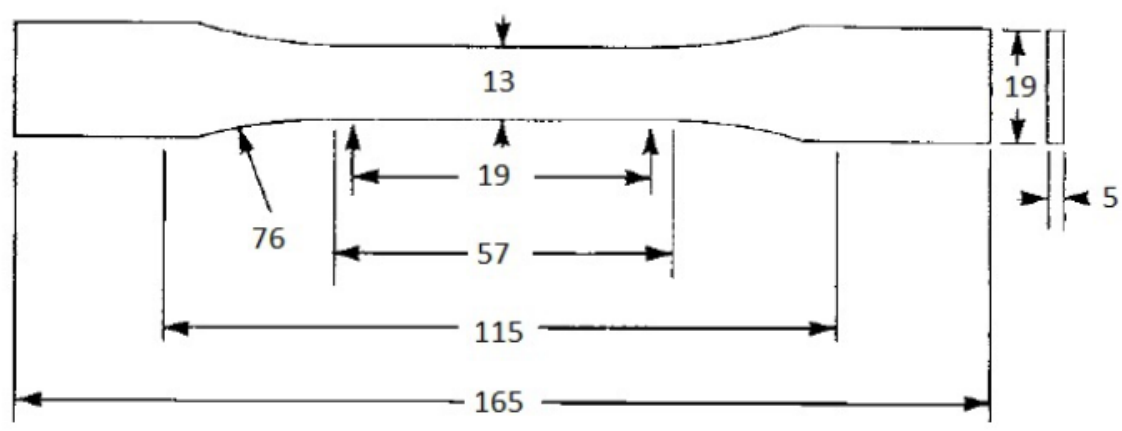

Note: All dimensions are in millimetres

Figure 4.9: ASTM International recommendation for plastic test specimens [36]

In an attempt to fabricate these specimens, many problems were encountered such as a brittle and fragile finish, high void content and the formation of a meniscus in the liquid resin causing curvature on the surface of the specimen. Reinforcing the neat resin was investigated; however it was decided that the fibres would resist creep and mask the viscoelastic nature of the epoxy. As a result of these manufacturing difficulties, it was decided to diverge from the recommended rectangular cross section. For simplicity of manufacturing and to reduce potential edge effects, the final specimen design has a circular cross section and was cast from a standard drinking straw with approximate dimensions shown in Figure 4.10.
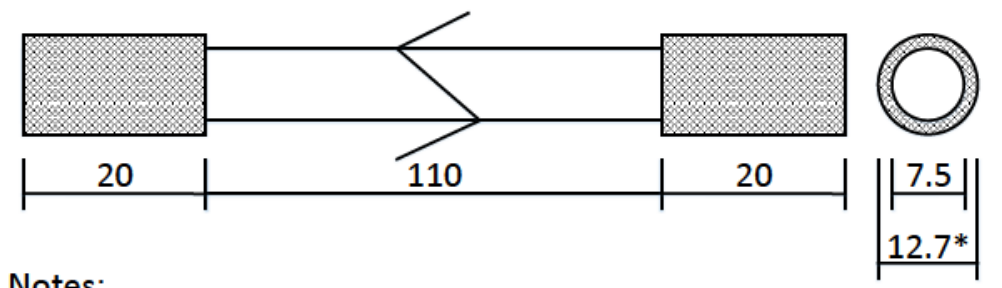

Notes:

- This drawing is not to scale

- Dimensions may vary up to $10 \%$

- * Fixed dimension $(1 / 2$ inch diameter $)$

Figure 4.10: Desired dimensions for custom specimens using neat resin

The specimen uses the same epoxy used in the Katana wing spar, the MGS L285/H287 epoxy system [2]. The following procedure outlines the manufacturing process:

1. Read and follow all safety procedure outlined in the MSDS provided by MGS. 
2. Prepare specimen molds by blocking one end of the drinking straw and place it in a container which will keep it in an upright position. In this situation, a portion of a hot glue stick and a paper cup was used.

3. Measure equal part MGS L285 resin and H287 Hardner and thoroughly mix until resin has a uniform constancy. Note the approximate time.

4. Degas mixture for 5 minutes or until the majority of the bubbles have been removed. This will required a chamber and vacuum pump which is able to safely maintain a minimum of vacuum strength of $23 \mathrm{inHg}$ or $78 \mathrm{kPa}$. A dry type pump should be used to avoid contamination of the pumps lubricant and the exhaust should be vented into a fume hood or outside to avoid the inhalation of toxic fumes.

5. Inject resin into molds using a syringe while being careful to limit the aeration of the liquid mixture.

6. Degas the mixture filled molds at 30 minute intervals for the remainder of the working time outlined by MGS. At the end of each interval vent the degassing chamber to remove the heat generated during the cure process. If the degassing chamber is not regularly vented or cooled, the temperature will drastically increase and damage the molds which will render the specimens unusable.

7. Allow the sample to completely cure, observations showed 2 to 3 times the recommended 24 hour cure time is required to fully cure the specimens when encased in the plastic drinking straws.

8. Bond aluminium sleeves on each end of specimen using freshly mixed resin. These sleeves are used to engage the gripping mechanism and were manufactured to the specimen diameter.

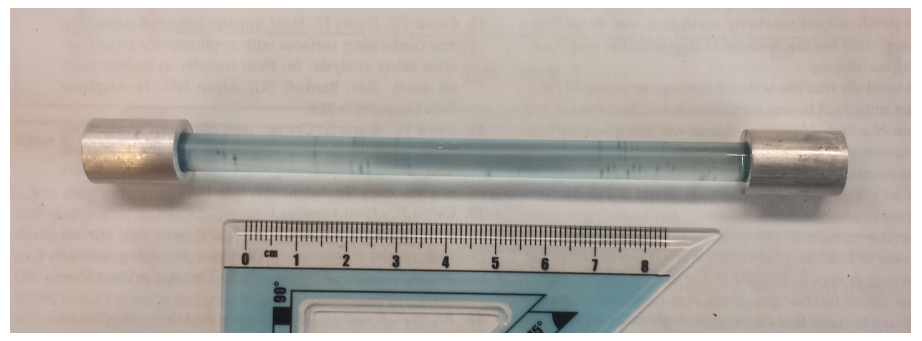

Figure 4.11: Completed epoxy specimen used in the creep and relaxation tests 
The following is the procedure required to safely load each specimen into the testing apparatus:

1. If the apparatus has been previously powered down, turn on the bi-polar power supply and toggle the blue switch to the ON position to supply power to the various components. For elevated test temperatures, turn oven power dial to low and adjust thermometer dial to desired temperature. Allow 30 minutes for sensors reach their operating temperature if starting cold. This can be verified by ensuring each sensor is reading a steady state value.

2. Start LabVIEW Program using the play button in the top left corner. This will activate the control of the linear actuator.

3. Measure specimen length and diameter and record in appropriate location in the LabVIEW interface.

4. Set test time, number of data points, desired load or displacement as well as file path for the data sheet.

5. Load specimen into apparatus and allow sufficient time for it to reach the desired temperature. Use insulated gloves if temperatures are above $50{ }^{\circ} \mathrm{C}$.

6. Run test using the "Start Test" button.

Once the test data has been collected, the viscoelastic modulus of the epoxy resin is determined. Once found, they are used to formulate a working life of the composite spar. The results and analysis of this data is outlined in the following chapter. 


\section{Chapter 5}

\section{Results and Analysis}

Creep and relaxation tests were performed on the epoxy specimens and the results and subsequent analysis are outlined in this chapter. It begins by outlining the long term viscoelastic behaviour of the material through the generation of a master curve. This is followed by the analysis of the composite spar using the elastic and the steady state viscoelastic modulus using the theory presented in Chapter 3.

\subsection{Viscoelastic Response of the Epoxy Resin}

Based on the information outlined in Section 3.2, tests were conducted using the experimental setup described in Chapter 4. The result from these tests were analysed using a custom MATLAB algorithm. The elastic modulus was first measure using eight specimens at temperatures between $35^{\circ} \mathrm{C}$ and $60{ }^{\circ} \mathrm{C}$. Based on these test elastic modulus was determined to be between 850-1350 MPa with an average of $1100 \mathrm{MPa}$. Possible reasons for this large variation are from difficulties measuring moduli due to the phase delay between the load application and the materials response described in Section 2.3. Figure 5.1 is an illustration of actual stress-strain data from one of the specimens. 


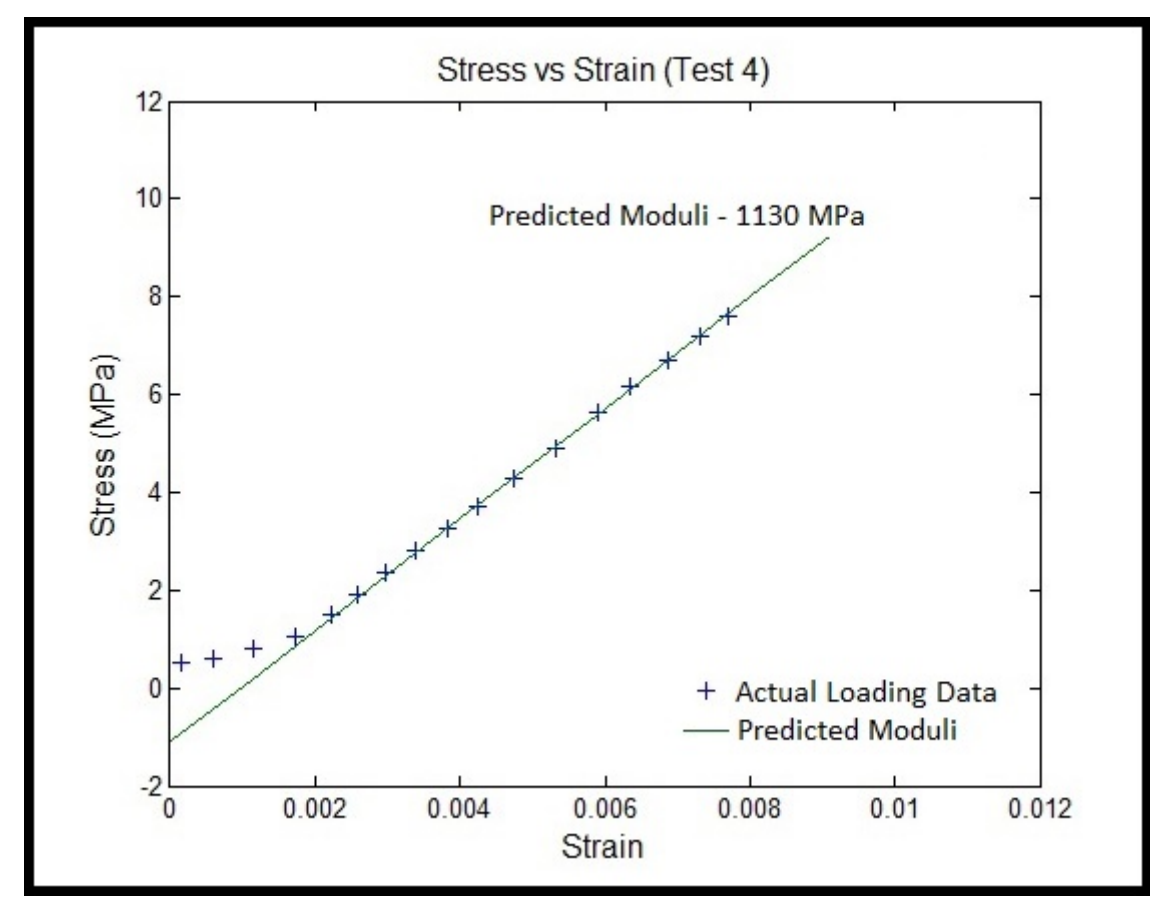

Figure 5.1: Loading data used to measure the initial modulus of the specimen

In addition to the variation, the measured elastic modulus was one third of the manufacturer's stated $3300 \mathrm{MPa}$. There are two contributing factors which may have lead to this discrepancy. Firstly the H287 hardener had been previously opened and at the time of use was many months past its expiry date. This would have lead to a reduced amine content resulting in a decrease in the cross linking power of the hardener. As a result, the fully cured specimen will have different characteristics than what is described by the manufacturer. Further tests should be conducted using varying degrees of fresh and expired hardener to validate as well as quantify the effect of this amine reduction. The second reason for a decrease in the measured stiffness is that this test set up is not equipped to measure the short term response of the material and was unable to capture the level of relaxation during the loading cycle. The short term response of this material is out of the scope of this thesis; however tests should be conducted to fully quantify it's mechanical response. To observe this short term response, tests should be conducted at a reduced operating temperature with the application of the time temperature superposition principle described in Section 3.2.2. With the initial loading characterized, the long term response of the epoxy can be analysed.

To create the master curves and governing equations that dictates the response of the 
Table 5.1: Comparison of temperature to respective time shift for epoxy resin given a $25^{\circ} \mathrm{C}$ reference temperature and $270 \frac{\mathrm{kJ}}{\mathrm{mol} \cdot \mathrm{K}}$ activation energy

\begin{tabular}{|c|c|}
\hline Temperature $\left({ }^{\circ} C\right)$ & Time shift $a_{t}(\log (s))$ \\
\hline 0 & 4.33 \\
\hline 10 & 2.51 \\
\hline 20 & 0.81 \\
\hline 25 & 0 \\
\hline 30 & -0.78 \\
\hline 40 & -2.27 \\
\hline 50 & -3.66 \\
\hline 60 & -4.97 \\
\hline 70 & -6.21 \\
\hline
\end{tabular}

*Note: Negative values indicate a shift into the future height

material, both creep and relaxation data were used. Six creep tests were done using a load of approximately $300 \mathrm{~N}$ and six relaxation tests were performed using a strain of approximately 0.01 . Each test was conducted at a different temperature between $35^{\circ} \mathrm{C}$ to $60^{\circ} \mathrm{C}$. The load, strain and temperature range was selected based on the current capabilities of the equipment which will be described in Chapter 6. For a longer service life prediction, tests were planned using the William Landel Ferry equation at temperatures above the manufactured specified glass transition point. Two tests were conducted above $80^{\circ} \mathrm{C}$ however this data was not used due to the inability to properly apply load to the specimen. These tests did qualitatively confirm the glass transition point as drastic changes in stiffness and ductility were observed. Table 5.1 lists the time temperature shifting factor $a_{t}$ for a $25^{\circ} \mathrm{C}$ reference temperature using a $270 \frac{\mathrm{kJ}}{\mathrm{mol} \cdot \mathrm{K}}$ activation energy.

Once the master curve has been created by shifting the data, a Prony series was fit to the data using non linear regression. Due to large variations in the limited data set, only a one term Prony series was able to converge for both the creep and relaxation test. The equations that represent the compliance and the relaxation functions, $J(t)$ and $Y(t)$, are found in Equations 5.1 and 5.2 and are compared with the test data 
shown in Figures 5.2 and 5.3. It should be noted that these plots do not show the short term response of the material as it is out of the scope of this thesis; however this should be performed at a later date to fully characterize the MGS L285/H287 epoxy system.

$$
\begin{gathered}
J(t)=\left[648-646 \cdot \exp \left(^{-t} / 1.73 E 6\right)\right]^{-1} \\
Y(t)=697-677 \cdot \exp \left(^{-t} / 6.26 E 4\right)
\end{gathered}
$$




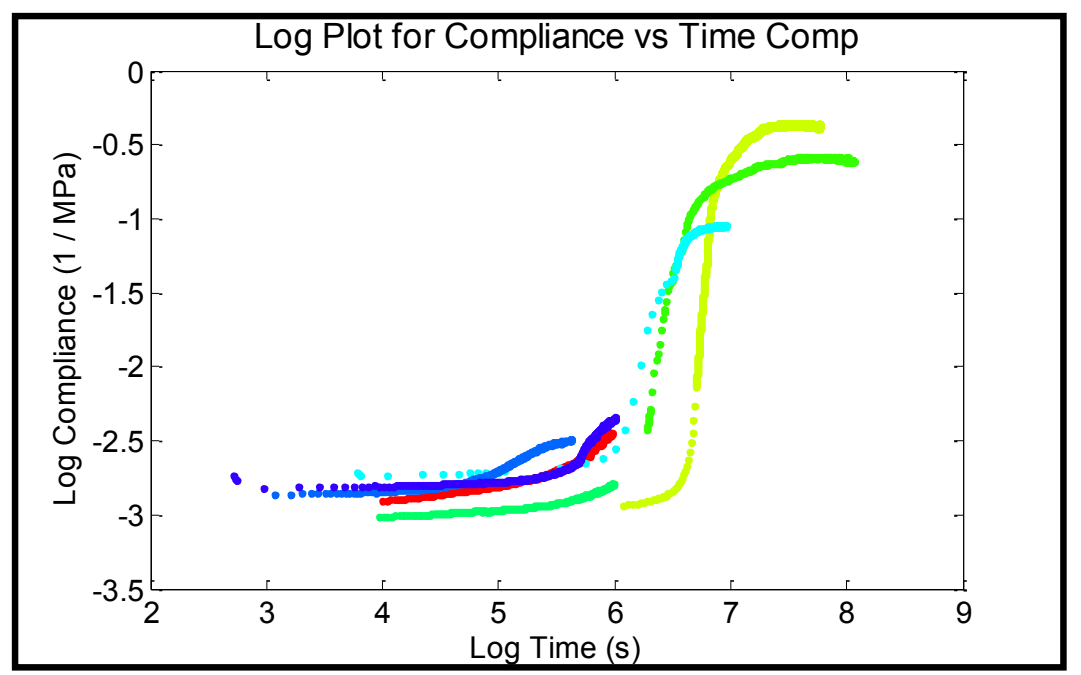

A - Tests distinguished by colour

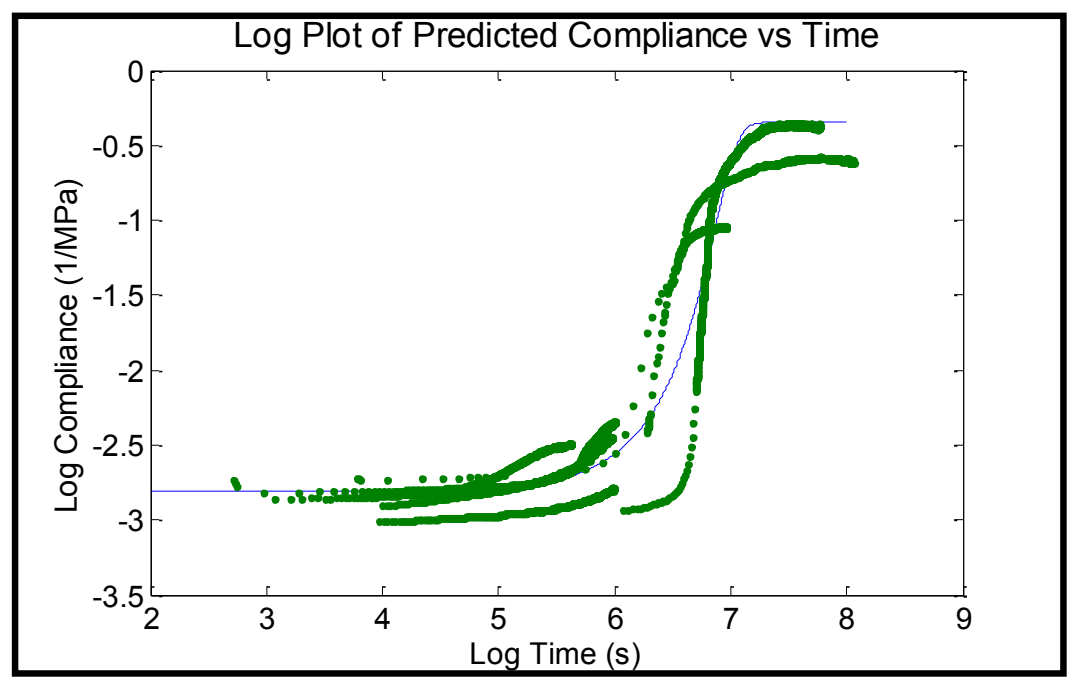

B - Compared to MATLAB fit

Figure 5.2: Master creep compliance curve generated for a reference temperature of $25^{\circ} \mathrm{C}$ 


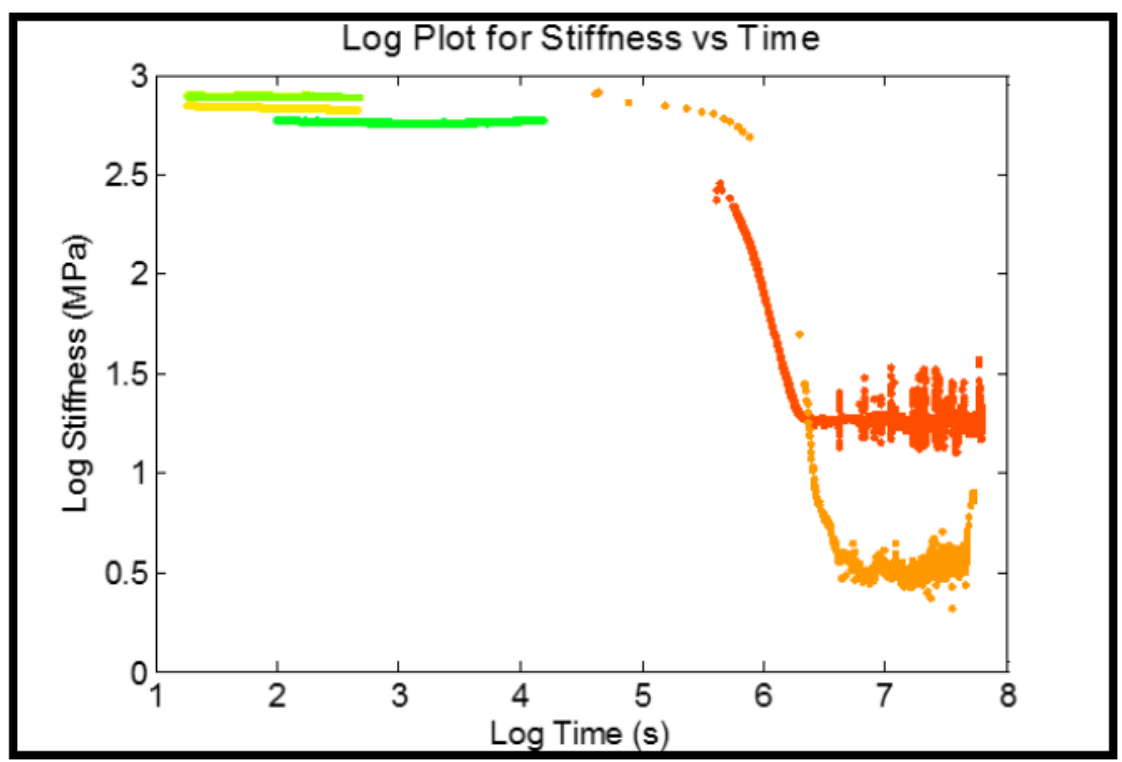

A - Tests distinguished by colour

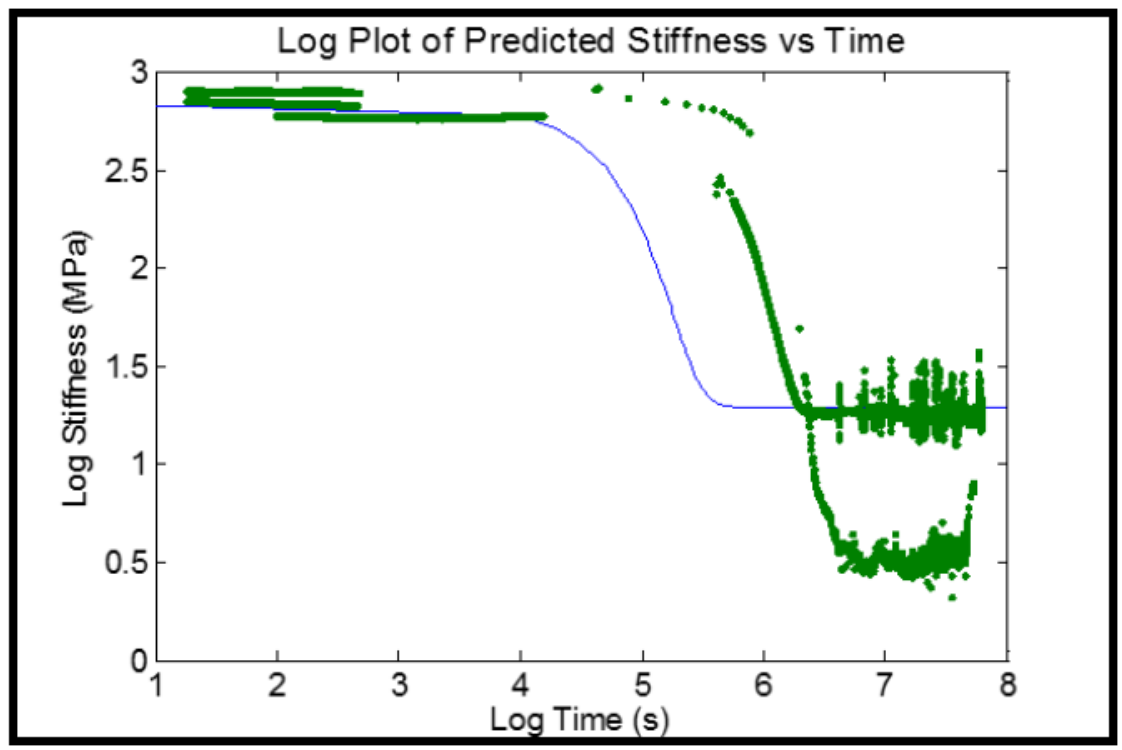

B - Compared to MATLAB fit

Figure 5.3: Master relaxation stiffness curve generated for a reference temperature of $25^{\circ} \mathrm{C}$ 
From inspection of the creep compliance function it can be concluded that the MGS epoxy system does adhere to the time temperature superposition principle and the long term response of the material can be determined. By examining Equation 5.1 it is apparent that it under predicts the initial modulus by $41 \%$ when compared to the $1100 \mathrm{MPa}$ determined earlier. The probable cause for this is that for the current test temperatures, there are no data points to represent the response before 100 seconds once the data has been shifted using the time temperature correlation as shown in Figure 5.2. The predicted time constant however does correlate to the data as shown in the Figure 5.2.

By examining the relaxation function in Figure 5.3, it is apparent that there is a correlation between the data as the trend is similar to that obtained through the creep test. In addition, the initial moduli of $697 \mathrm{MPa}$ in Equation 5.2 was similar to that determined by Equation 5.1. Unfortunately due to the limited data set the relaxation time constant determined through non linear regression did not correlate to the data as shown in Figure 5.3. The steady state stiffness of the MGS epoxy system was determined by taking the average value determined through Equations 5.1 and 5.2. Using this assumption the fully relaxed stiffness was determined to be approximately $10 \mathrm{MPa}$.

It is important to note that the activation energy is typically not provided by the manufacturer and was determined by performing the analysis over a wide range of values. An activation energy of $270 \frac{\mathrm{kJ}}{\mathrm{mol} \cdot \mathrm{K}}$ was selected as it provided the most sensible fit when performing nonlinear regression through the data. Figure 5.4 is an illustration of the final fit compared to the data for various activation energies. 


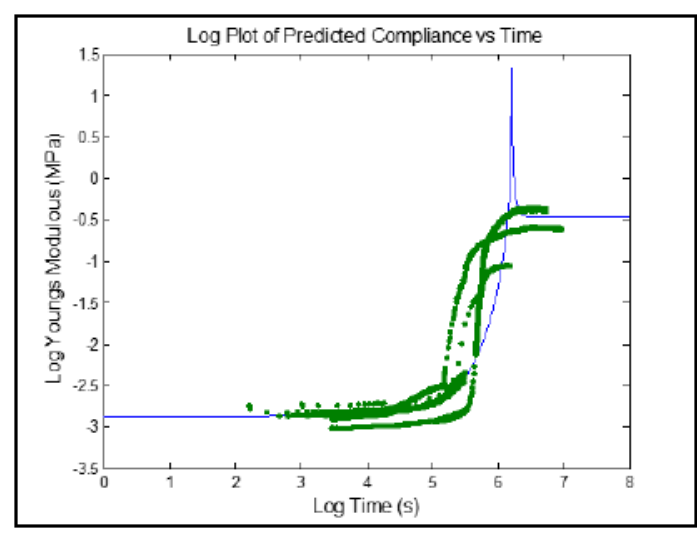

Activation Energy - $200 \mathrm{Kmol} / \mathrm{K} \mathrm{K}$

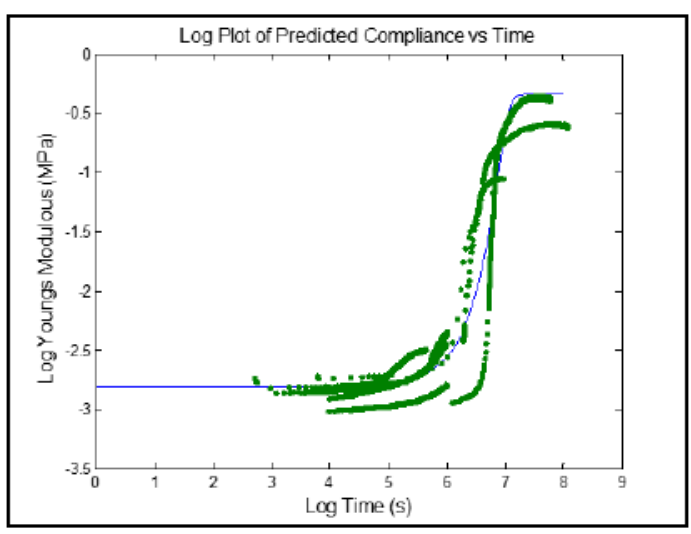

Activation Energy - 270 Kmol/ $/ \mathrm{K}$

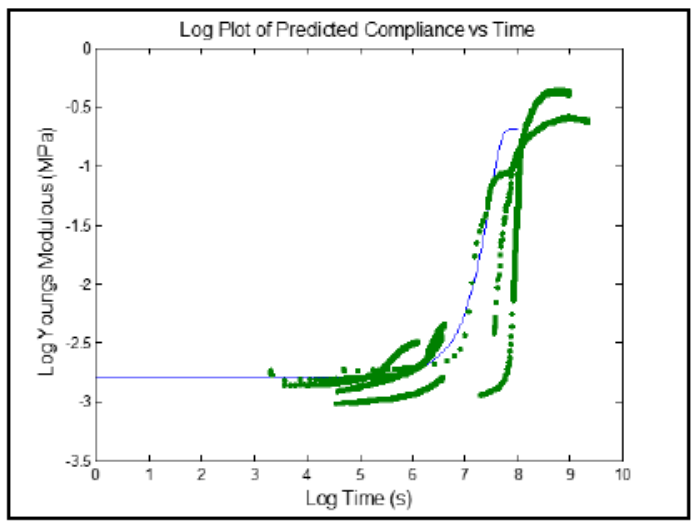

Activation Energy - 350 Kmol $/ \mathrm{k} \mathrm{K}$

Figure 5.4: Master creep compliance curve generated using various activation energies

Based on Figures 5.2 and 5.3, it is apparent that the onset of significant increase in creep compliance (decrease in stiffness) will occur around 6 decades of second (approx. 11 days) and will be at its maximum before 7 decades of seconds (approx 16 week). Similar results were observed during relaxation tests where the stiffness begins to relax around 6 decades and is fully relaxed before 7 decades. A comparison of the creep and relaxation data normalized for stiffness is found in Figure 5.5. 


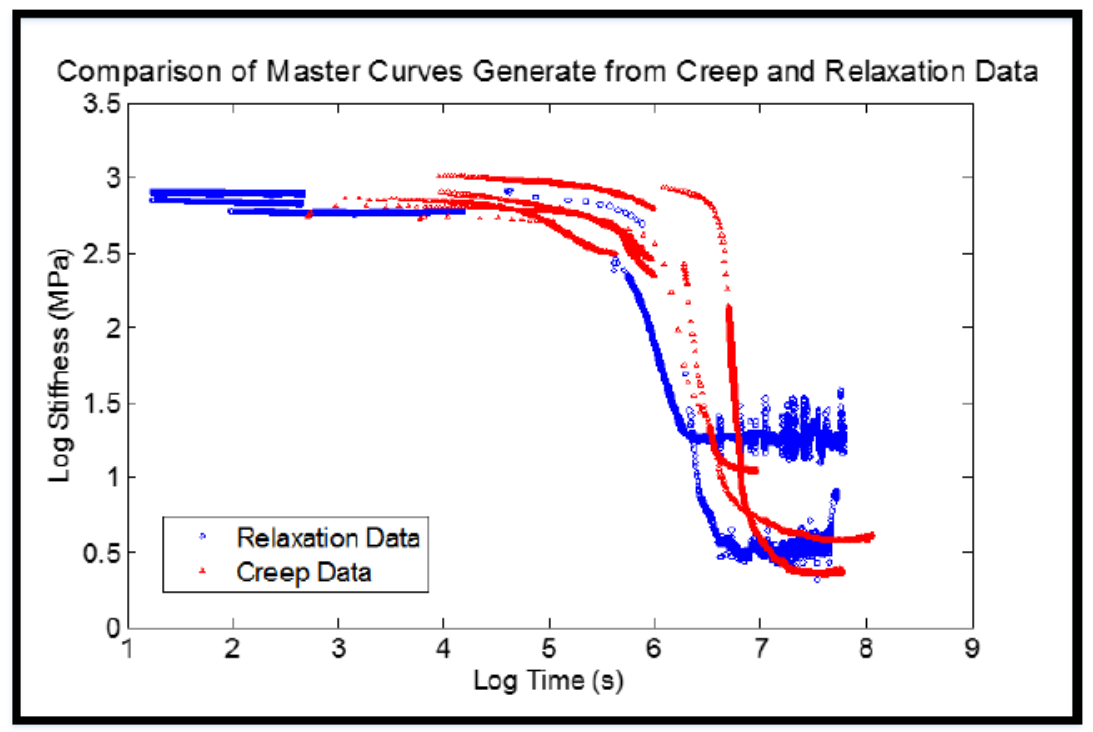

Figure 5.5: Comparison of master curves generated for $25^{\circ} \mathrm{C}$ from creep and relaxation data normalized for stiffness

This is contrary to the theory presented in Section 3.2 which states that these will occur at different rates. The probable cause of this would be the use of a limited data set and due to limitations with the testing apparatus such as with temperature control. Figure 5.6 depicts the scatter when creating the compliance master curve using the instantaneous test temperature instead of average test temperature to determine the shifting factor. 


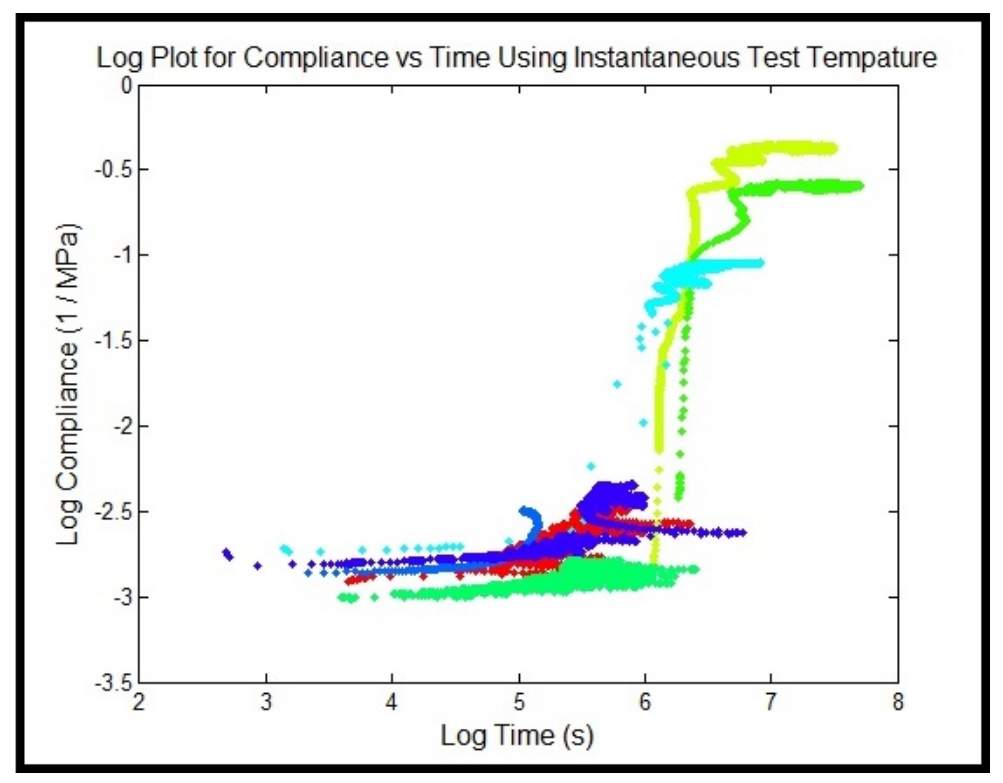

Figure 5.6: Creep compliance master curve using instantaneous temperature to determine shift factor

Unfortunately the current testing apparatus is unable to properly control its load in the range required and as a result accurate recovery data is not currently available. This limitation and potential improvement which can be made to obtain this data will be explained in Chapter 6. Although the Katana aircraft is rarely in the air for more than a few hours at a time, safe design practise should assume the matrix has reached its relaxed steady state stiffness. As a result, recovery data is not required for this service life prediction; however it should be measured in the future to fully characterize the MGS epoxy system. The following section will outline the response of the composite wing spar and its implications on service life.

\subsection{Response of Composite Wing Spar}

Based on the method presented in Section 3.3, an algorithm to perform the modified CLT analysis was created using MATLAB. As experimental data is not available for this aircraft, elastic properties were used and compared to an expected range to validate this algorithm. Before the structural response can be analysed, a stacking sequence of the composite spar must be determined. 


\subsubsection{Determination of Ply Layup}

To determine the exact layup of the composite I-beam, either manufacturer drawings are required or destructive testing must be performed. Since neither of these options were available, basic assumptions were made based on expected loads and the geometry of the beam. Due to the geometric nature of I-beams, the flanges at the top and bottom carry mostly tensile and compressive loads while the centre web carries shear loads.

Since the flanges carry tensile and compressive load, it is desirable to have fibres in the longitudinal or x-direction. To account for other loads during flight, smaller layers in the centre of the plate oriented at $\pm 60^{\circ}$ have been added for strength in all directions. From visual inspection of the spar in Figure 1.4, the flanges are assumed to be manufactured from carbon fibre. Finally, to eliminate the coupling loads which can warp the spar, a symmetric layup is assumed as with most practical composite beams. Based on these assumptions, the assumed flange layup is shown in Figure 5.7.

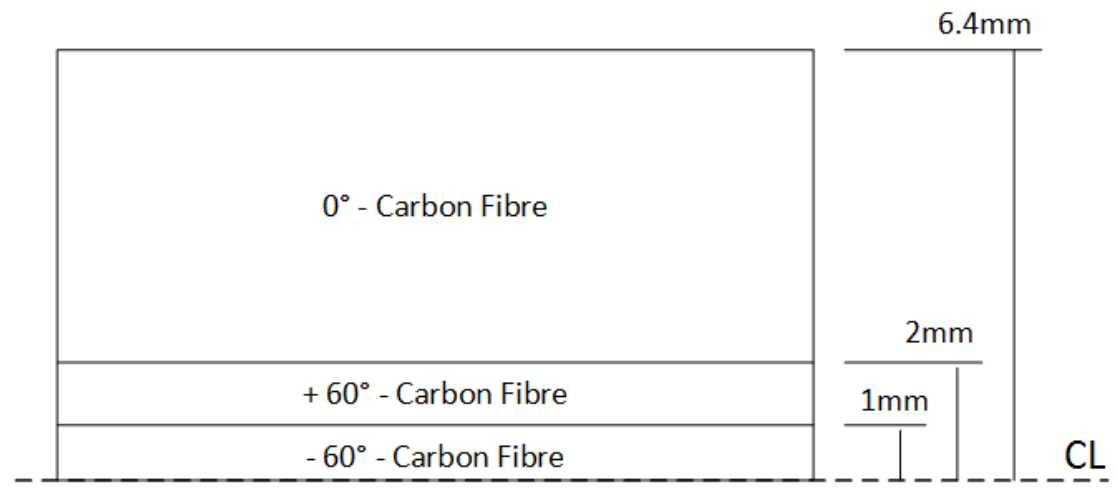

Note: This drawing is not to scale and is for representational purposes only

Figure 5.7: Assumed stacking sequence for the top and bottom flanges

In beams of this geometry, the web typically carries shear loads induced by the tensile and compressive loads of the flanges. When examining shear forces the resultant forces are assumed to be at applied at $\pm 45^{\circ}$. As a result, it is expected that the majority of the web will consist of fibres oriented in those directions and would be placed closer to the mid-plane. As with the flange, other loads will be accounted for by placing a layer of fibres oriented at $0^{\circ}$ and $90^{\circ}$ at each end. Based on these assumptions, the assumed web layup is represented by Figure 5.8. 


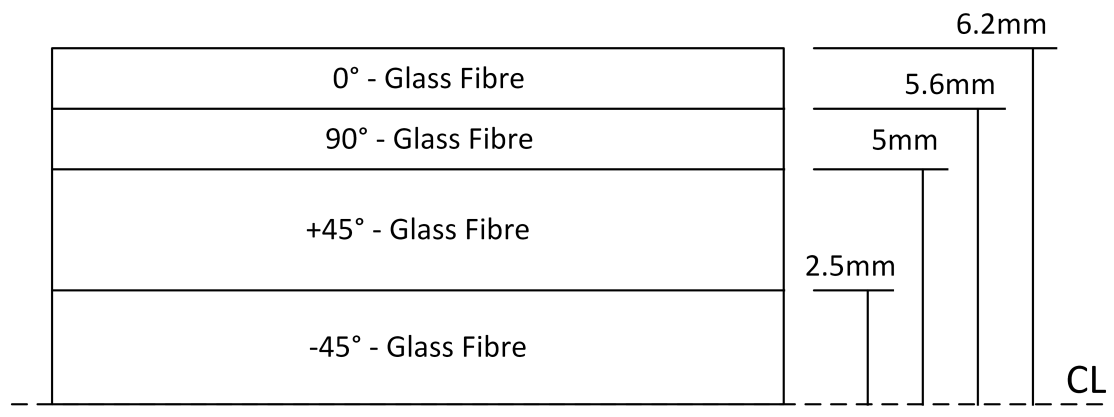

Note: This drawing is not to scale and is for representational purposes only

Figure 5.8: Assumed stacking sequence of the web

Using these assumed stacking sequences, the elastic response of the spar is determined and used validate the MATLAB algorithm.

\subsection{Elastic Analysis of Composite Wing Spar}

To confirm the MATLAB algorithm, the response of the spar was determined using the elastic properties of the epoxy matrix. The loading experienced by the spar is based on an ideal elliptical loading profile encountered during steady level flight at its maximum take off weight. This is an appropriate first approximation as the Diamond Katana was modelled after a Diamond HK36 Dimona motorglider which is known for its aerodynamic characteristics [2]. Important properties regarding the aircraft and the raw materials are outlined in Tables 5.2 and 5.3.

Table 5.2: Important Dimensions of the Diamond DA-20 Katana

\begin{tabular}{|l|l||l|l|}
\hline Wing Span & $10 \mathrm{~m}$ & Wing Chord & $1.09 \mathrm{~m}$ \\
\hline Flange Width & $74 \mathrm{~mm}$ & Web Width & $118.9 \mathrm{~mm}$ \\
\hline Flange Thickness & $14.8 \mathrm{~mm}$ & Web Thickness & $12.5 \mathrm{~mm}$ \\
\hline
\end{tabular}

Three criterion will be used for validation. The first characteristic to be examined is the deflection of the wing. Based on the assumptions presented in Section 3.3.1; the twist, vertical and horizontal deflection of the wing tip were determined to be $0.15^{\circ}, 5.6 \mathrm{~cm}$ and $5.5 \mathrm{~cm}$ from their original positions which are well within the 
Table 5.3: Elastic properties of the raw materials used to fabricate the composite wing spar [37-39]

\begin{tabular}{|l|c|c|c|}
\hline Material Property & $\begin{array}{c}\text { Glass Fibre } \\
\text { Interglas } \\
\text { E-Glass } 92145\end{array}$ & $\begin{array}{c}\text { Carbon Fibre } \\
\text { Hexcel } \\
\text { HexTow AS4 }\end{array}$ & $\begin{array}{c}\text { Epoxy } \\
\text { Martin G. Scheufler } \\
\text { L285/H287 System }\end{array}$ \\
\hline Elastic Modulus & $73 \mathrm{GPa}$ & $231 \mathrm{GPa}$ & $3.3 \mathrm{GPa}$ \\
\hline Elongation at Break & $1.9 \%$ & $1.7 \%$ & $5-6 \%$ \\
\hline Tensile Strength & $2000 \mathrm{MPa}$ & $4410 \mathrm{MPa}$ & $70-80 \mathrm{MPa}$ \\
\hline Compressive Strength & - & - & $120-140 \mathrm{MPa}$ \\
\hline Glass Transition Temperature & - & - & $90-95^{\circ} \mathrm{C}$ \\
\hline
\end{tabular}

Notes:

- Data collected from manufactures specifications

- Assumed f

expected ran

vertical

wing deflecti sould be

noted that tl provide

additional su

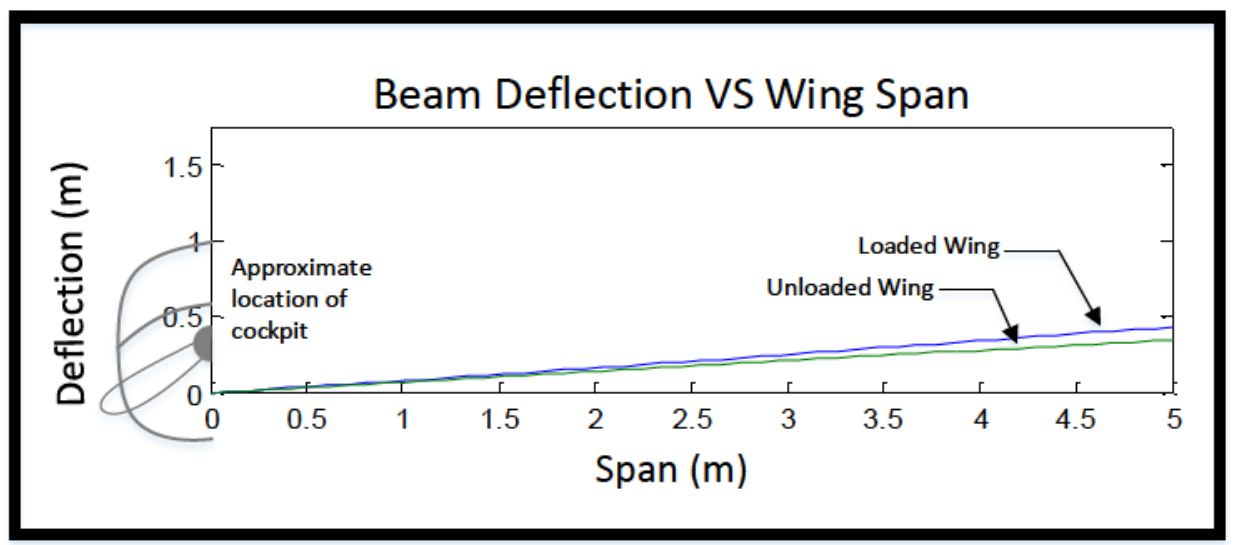

Figure 5.9: Graphical representation of the wing's vertical deflection during steady level flight, including a reference to the aircraft cockpit

The second method of validation is to examine the stress within the flanges and web 
and ensure they pass the Tsai-Hill and maximum strain failure criterion. For composite beams that comprise of both horizontally and vertically oriented components, the web and flange sections needed to be divided into quadrants to determine the stress at various locations. Figure 5.10 is an illustration of each quadrant within the I-beam. The minimum strength ratios for each section were determined and are presented in Table 5.4 .

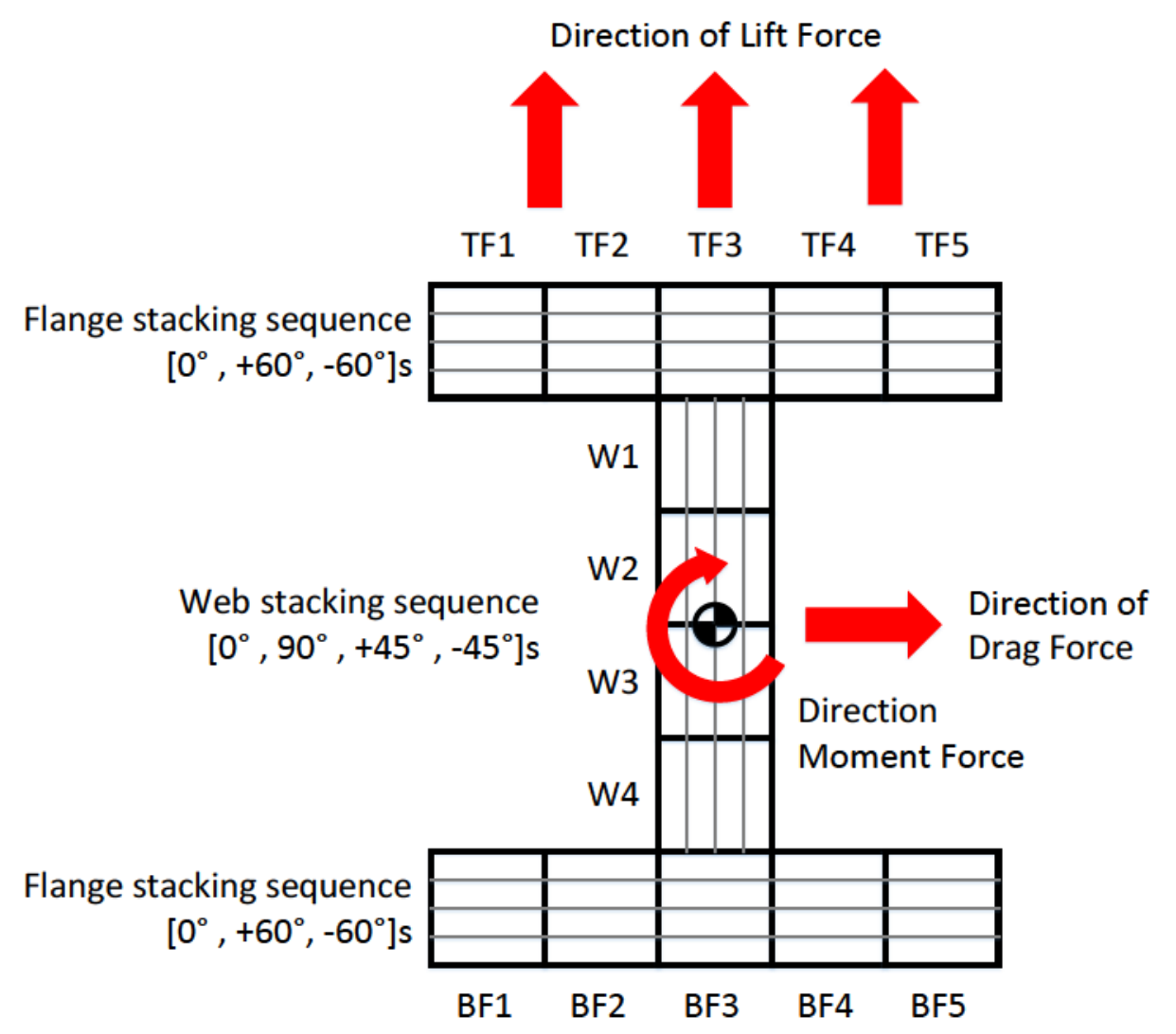

Note: Hatch pattern is used to show which components have vertically or horizontally oriented fibres and not the number of plies.

Figure 5.10: Quadrant breakdown of I beam for stress analysis

This analysis has shown that the Tsai-Hill strength ratios are strongly dependant on the amount of shear stress present in each ply as the shear strength is the limiting factor. It is for this reason we see the lowest strength ratios on the $\pm 60^{\circ}$ plies in the flanges and on the $0^{\circ}$ and $90^{\circ}$ plies in the web. In addition, the large strength ratios shown section W2 and W3 are a result of their close proximity to the neutral axis of 
Table 5.4: Table listing strength ratio of the weakest ply within each section of the I-beam using elastic properties and the Tsai-Hill and Maximum strain failure theory

\begin{tabular}{|c|c|c|}
\hline Section & $\begin{array}{c}\text { Minimum Strength Ratio } \\
\text { Tsai-Hill Theory }\end{array}$ & $\begin{array}{c}\text { Minimum Strength Ratio } \\
\text { Max Strain Theory }\end{array}$ \\
\hline TF1 & 8.1 & 8.5 \\
\hline TF2 & 10.1 & 9.4 \\
\hline TF3 & 12.8 & 10.9 \\
\hline TF4 & 11.4 & 10.0 \\
\hline TF5 & 9.2 & 9.1 \\
\hline \hline BF1 & 8.1 & 8.1 \\
\hline BF2 & 10.1 & 9.4 \\
\hline BF3 & 13.7 & 10.9 \\
\hline BF4 & 11.1 & 10.1 \\
\hline BF5 & 9.1 & 9.1 \\
\hline \hline W1 & 81 & 21 \\
\hline W2 & 636 & 63 \\
\hline W3 & 648 & 63 \\
\hline W4 & 82 & 21 \\
\hline
\end{tabular}

the I-beam where little to no stress is present.

The final method of validation is to ensure the I-beam will not buckle based on its elastic properties. Based on the analysis, it was determined that the critical buckling moment is $3.05 \mathrm{E} 5 \mathrm{~N} \cdot \mathrm{m}$ which provided a safety factor of 18.3 when compared to the expected moment at the root of the wing spar.

Based on the results presented above, the stresses within the composite spar fell within the requirements outlined in Section 3.5 as each ply had a strength ratio well above 2 . With the elastic properties confirmed, the viscoelastic characteristics of the wing spar can be examined. 


\subsection{Viscoelastic Analysis the Wing Spar}

To determine if the composite spar has failed using the material's viscoelastic properties, an analysis will be conducted using the fully relaxed matrix modulus during steady level flight. This is a valid assumption as thermosetting polymers creep and relax to a steady state stiffness as described in Section 3.2. This section outlines the steady state response of the wing spar and compares it to the initial values. This is followed by an analysis of the failure conditions outlined in Section 3.5.

\subsubsection{Viscoelastic Response During Steady Level Flight}

Using the same algorithm explained above, the matrix modulus was set to its fully relaxed value determined through the generation of the materials master curve. Using these assumptions, the variation in the beams effective axial and flexural stiffness when using the initial value to the fully relaxed value were less than $10 \%$. As a result there is little change in the wings steady state deflections when compared to it's initial condition. Table 5.5 outlines key differences between the response of a new and relaxed spar.

Table 5.5: Comparisons of the wing spars response using elastic and viscoelastic properties

\begin{tabular}{|l|c|c|c|}
\hline Modulus of Epoxy $(\mathrm{MPa})$ & 3300 & 1000 & 10 \\
\hline \hline Effect. Axial Stiffness $(\mathrm{N})$ & $1.40 \mathrm{E} 8$ & $1.31 \mathrm{E} 8$ & $1.27 \mathrm{E} 8$ \\
\hline Effect. Flexural Stiffness - x $\left(\mathrm{Nm}^{2}\right)$ & $5.65 \mathrm{E} 5$ & $5.42 \mathrm{E} 5$ & $5.32 \mathrm{E} 5$ \\
\hline Effect. Flexural Stiffness - y $\left(\mathrm{Nm}^{2}\right)$ & $5.56 \mathrm{E} 4$ & $5.58 \mathrm{E} 4$ & $5.51 \mathrm{E} 4$ \\
\hline Effect. Torsion Rigidity - xy $\left(\mathrm{Nm}^{2}\right)$ & $2.27 \mathrm{E} 5$ & $1.82 \mathrm{E} 5$ & $1.16 \mathrm{E} 5$ \\
\hline Vert. Wing Tip Deflection $(\mathrm{cm})$ & 5.6 & 5.8 & 5.9 \\
\hline Hor. Wing Tip Deflection $(\mathrm{cm})$ & 5.5 & 5.7 & 5.8 \\
\hline Wing Tip Angle of Twist $(\mathrm{deg})$ & 0.15 & 0.19 & 0.21 \\
\hline
\end{tabular}

These results are expected if the assumptions made in CLT are closely examined. As expected, the fibres carry the majority of the load in composite structure as the stiffness of glass and carbon fibres are approximately 20 and 80 times larger than that of the epoxy respectively. By setting the matrix modulus to its relaxed value, 
the algorithm shows that the fibres could support the full load assuming it maintains its shape and that other phenomena such as delamination, debonding or interply buckling do not occur. The effects of these phenomena using viscoelastic data is out of the scope of this thesis and should be examined in future studies. It is also important to note that as the matrix relaxes, a decrease in web stress is expected as the load is redirected to the stiffer flanges. Using the data for a viscoelastic wing spar, failure analysis can be conducted using the three criterion.

\subsubsection{Viscoelastic Failure Analysis}

If the ultimate strengths of the epoxy are assumed to not change with time, a fully relaxed spar will pass the Tsai-Hill failure criteria. This is an invalid assumption as one would expect a decrease matrix strength; however no data has been gathered on this due to height constrictions with the current test setup. To test the robustness of this criteria, it was assumed that the ultimate strength of the matrix decreases over time and the results are found in Table 5.6.

Based on the results presented in Table 5.6, it would approximately require a 99.9\% decrease in the matrix strength for failure to occur. An important observation when comparing the initial condition of Table 5.4 to the second column of Table 5.6 is the increase in strength ratio as the matrix relaxes. This is due to a transfer of stress from the shear direction to the fibre-wise direction. As a result when the matrix is fully relaxed the shear load within each ply are at a minimum since the fibres are carrying most of the load. As with the elastic case, the matrix shear strength is the limiting factor and will be the ultimate cause of failure. When analysing the results of the web section, it is apparent that the strength ratios increase by approximately an order of magnitude. This is caused from the transfer of load within the web to the top and bottom flanges. Based on this analysis it can be concluded that the wing spar will pass the Tsai-Hill failure criteria using the relaxed characteristic of the epoxy.

The wing spar also passes the maximum strain failure theory using the viscoelastic properties of the epoxy. Since the maximum sustainable strain of fibres are approximately 3 times lower than that of the epoxy matrix, the properties of the fibres will be the limiting condition in both the longitudinal and transverse directions. Based on the analysis, the maximum strain observed by the composite spar using viscoelastic 
Table 5.6: Table listing strength ratio of the weakest ply within each section of the I-beam using the relaxed properties, Tsai-Hill failure theory and a varying ratio of epoxy strength

\begin{tabular}{|c|c|c|c|c|}
\hline Section & $100 \%$ Strength & $10 \%$ Strength & $1 \%$ Strength & $0.1 \%$ Strength \\
\hline TF1 & 78 & 77 & 61 & 0.8 \\
\hline TF2 & 114 & 112 & 87 & 0.9 \\
\hline TF3 & 160 & 157 & 116 & 1.2 \\
\hline TF4 & 330 & 323 & 101 & 1.0 \\
\hline TF5 & 337 & 325 & 83 & 0.9 \\
\hline \hline BF1 & 72 & 70 & 61 & 0.8 \\
\hline BF2 & 103 & 101 & 87 & 0.9 \\
\hline BF3 & 160 & 157 & 116 & 1.2 \\
\hline BF4 & 280 & 274 & 101 & 1.0 \\
\hline BF5 & 337 & 325 & 81 & 0.8 \\
\hline \hline W1 & 901 & 861 & 530 & 7.2 \\
\hline W2 & $7.91 \mathrm{E} 3$ & $7.55 \mathrm{E} 3$ & $4.18 \mathrm{E} 3$ & 54 \\
\hline W3 & $7.91 \mathrm{E} 3$ & $7.55 \mathrm{E} 3$ & $4.28 \mathrm{E} 3$ & 55 \\
\hline W4 & 900 & 861 & 534 & 7.3 \\
\hline
\end{tabular}

properties is less than $12 \%$ of the maximum allowable strain specified by manufacturer. The strength ratios based on the maximum strain failure condition are found in Table 5.7. This table shows that there is little change in the strength ratios when comparing the results using the initial and relaxed matrix moduli.

Since the top flange will experience a compressive load, stability analysis was performed to ensure buckling will not occur as explained in Section 3.5. Based on this analysis and the assumptions made in the classical lamination theory, local buckling of the flange will not occur even though the critical buckling moment is $42 \%$ lower when assuming a fully relaxed matrix. Table 5.8 lists the critical moment for various moduli, the safety factor as well as the maximum applied moment during steady level flight. Using engineering judgement, these results must be experimentally verified as the assumption made for CLT may not be valid. Possible phenomena to invalidate the 
Table 5.7: Table listing strength ratio of the weakest ply within each section of the I-beam when compared to the maximum allowable elongation of the fibres using relaxed properties

\begin{tabular}{|c|c|c|c|}
\hline Section & $\mathrm{E}=3300 \mathrm{MPa}$ & $\mathrm{E}=1000 \mathrm{MPa}$ & $\mathrm{E}=10 \mathrm{MPa}$ \\
\hline $\mathrm{TF} 1$ & 8.5 & 7.9 & 7.8 \\
\hline TF2 & 9.4 & 9.1 & 8.9 \\
\hline TF3 & 10.9 & 10.4 & 10.1 \\
\hline TF4 & 10.0 & 9.8 & 9.4 \\
\hline TF5 & 9.1 & 8.8 & 8.6 \\
\hline \hline BF1 & 8.1 & 7.6 & 7.4 \\
\hline BF2 & 9.4 & 9.1 & 9.0 \\
\hline BF3 & 10.9 & 10.4 & 10.1 \\
\hline BF4 & 10.1 & 9.6 & 9.5 \\
\hline BF5 & 9.1 & 8.7 & 8.5 \\
\hline \hline W1 & 21 & 21 & 20 \\
\hline W2 & 63 & 61 & 60 \\
\hline W3 & 63 & 61 & 60 \\
\hline W4 & 21 & 21 & 20 \\
\hline
\end{tabular}

assumption are inter-ply or local buckling of the individual fibres and delamination or debonding of the plies.

\subsection{Summary of Service Life Prediction Using Vis- coelastic Properties}

Using results presented in this chapter, failure will not occur based on a relaxation of epoxy stiffness and strength. As a result, it can be assumed failure will occur based on the typical modes observed by composite materials such as delamination or damage to the fibres and matrix. This analysis has shown it is possible that the Diamond DA-20 Katana has an unlimited service life as the current design has a 
Table 5.8: Comparisons of the critical buckling moment for local buckling of the compressive flange to occur and their respective safety factor

\begin{tabular}{|c|c|c|c|}
\hline Epoxy Modulus & Critical Moment & Root Moment & Safety Factor \\
\hline $3300 \mathrm{MPa}$ & $3.05 \mathrm{E} 5 \mathrm{Nm}$ & $1.67 \mathrm{E} 4 \mathrm{Nm}$ & 18.3 \\
\hline $1000 \mathrm{MPa}$ & $2.27 \mathrm{E} 5 \mathrm{Nm}$ & $1.67 \mathrm{E} 4 \mathrm{Nm}$ & 13.6 \\
\hline $10 \mathrm{MPa}$ & $1.76 \mathrm{E} 5 \mathrm{Nm}$ & $1.67 \mathrm{E} 4 \mathrm{Nm}$ & 10.5 \\
\hline
\end{tabular}

large safety margin and that the fibres are capable of carrying the full load during flight. Currently the assumptions made while performing the buckling analysis are in question and as a result experimental tests must be performed to validate the compressive response of a composite flange. To determine a more accurate time to failure, scaled composite I-beams should be tested in bending and the time required for phenomena such as inter-ply buckling or debonding to occur should be observed. In addition, dynamic tests should be conducted as this would better simulate actual flight conditions and test for it's fatigue response. The following chapter will briefly outline possible modifications to the testing apparatus as well as further tests that should be conducted. 


\section{Chapter 6}

\section{Potential Improvements and Future Work}

Based on the experience gained during the research process, various improvements should be made to the equipment to allow for a broader range of testing conditions. This section will outline the limitations of the current setup, improvements to be considered and finally the tests required to further validate the results of this thesis.

\subsection{Limitations of the Current Test Setup}

As described in Section 4.1, in the early stages of design it was decided that a decommissioned undergraduate equipment designed to measure the creep in lead could be modified to meet our research needs. Figure 6.1 is an illustration of the decommissioned and current setup. 

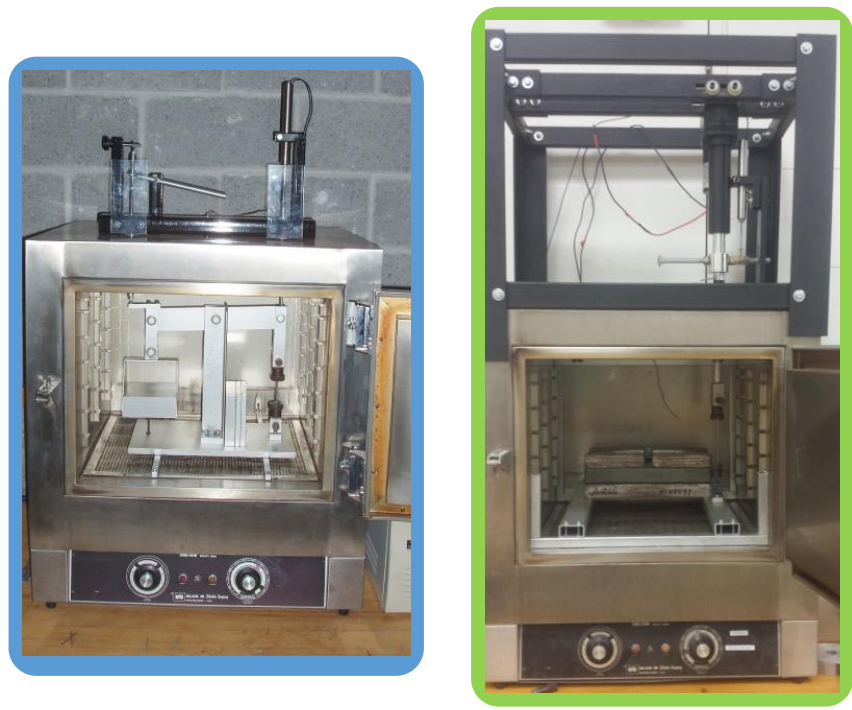

Figure 6.1: Comparison of past test equipment for lead, on the left, with the new equipment for polymers on the right

This was determined to be a suitable candidate as it still had many of the required sensors and since the oven was intended for lead which has a low melting point. As a result the oven could accurately maintain lower temperature when compared to typical creep equipment for aluminium or other metals. Although ASTM International recommends the use of dead weight for creep tests as described in Section 4.1, an electrical actuator and in line load cell was used instead of the existing lever system. This allows for greater diversity of tests and an easy implementation of the relaxation test. After the modifications were implemented and tests had commenced, it was apparent that the oven has four major limitations which are outlined below:

1. Even with structural reinforcement in key areas, it is unable to sustain loads above $500 \mathrm{~N}$ (100 lb) which drastically limits the size and characteristics of the test specimen. Using the current specimen dimensions and MGS specifications, the maximum applicable load is approximately $1 / 6$ of the specimen's tensile strength. As a result, this apparatus is unsuitable to test stronger composite specimens and is unable to test for yield and failure characteristics of the neat resin.

2. Although designed for lower temperatures, the oven is still unable to accurately maintain temperatures under $70^{\circ} \mathrm{C}$. Currently, temperatures above $30{ }^{\circ} \mathrm{C}$ are 
maintained using large steel thermal masses which limits the ability to select a desired temperature. In addition to this, the temperature controls are built into the oven and are separate from the LabVIEW interface which limits the ability to reliably adjust the temperature during a test.

3. The current height of the heated compartment limits the maximum creep elongation. Using current specimen dimensions, this apparatus is unable to perform a creep test beyond $30 \mathrm{~mm}$ as the gripping mechanism will exceed the oven dimensions.

4. The lack of viewing areas limits the possibility of using image processing to view multi-axis deformations to determine coupling characteristics such as the Poisson's ratio.

The following section will outline the design parameters required for a new oven to address all these issues.

\subsection{General Parameters for Custom Oven}

To address the problems stated above, the following design solutions have been conceived:

1. The structure will consist of a shell using steel square tube as it possesses good strength characteristics in addition to being cost effective. It must act as a rigid body by withstanding the maximum actuating load of $2500 \mathrm{~N}$ with little deflection. This will ensure an accurate reading of the specimen displacement. Considerations must be made for multiple attachment points to allow for various test configurations such as for tensile, compressive, and bending tests. The height of the box must also be adequate to allow for creep rupture of the specimen.

2. The heat source or element must be controlled through LabView using a switching relay in the same manner as the electric actuator. The heating elements selected must also be designed for low to moderate temperatures. Ideally they should be convective in nature and should be able to quickly reach their operating temperature. This will allow LabVIEW to maintain the oven temperature 
through short cycles which will increase the ovens ability to maintain a certain temperature. In addition, this allows for the possibility to adjust the temperature throughout a test to determine characteristics such as the glass transition point.

3. As low to moderate temperatures are employed, a wider range of materials can be used to construct the insulated portion of the oven. Potential materials include wood boards for the walls and stone wool for insulation as they both have auto ignition temperatures above $300^{\circ} \mathrm{C}$ and are reasonably priced when compared to steel plates and ceramic insulation.

4. To monitor the coupling characteristics of the material, a camera, glass window and grid markings should be installed on each axis to monitor the deformation and allow for image processing.

Figure 6.2 is a conceptual drawing of the oven incorporating the required specifications. 


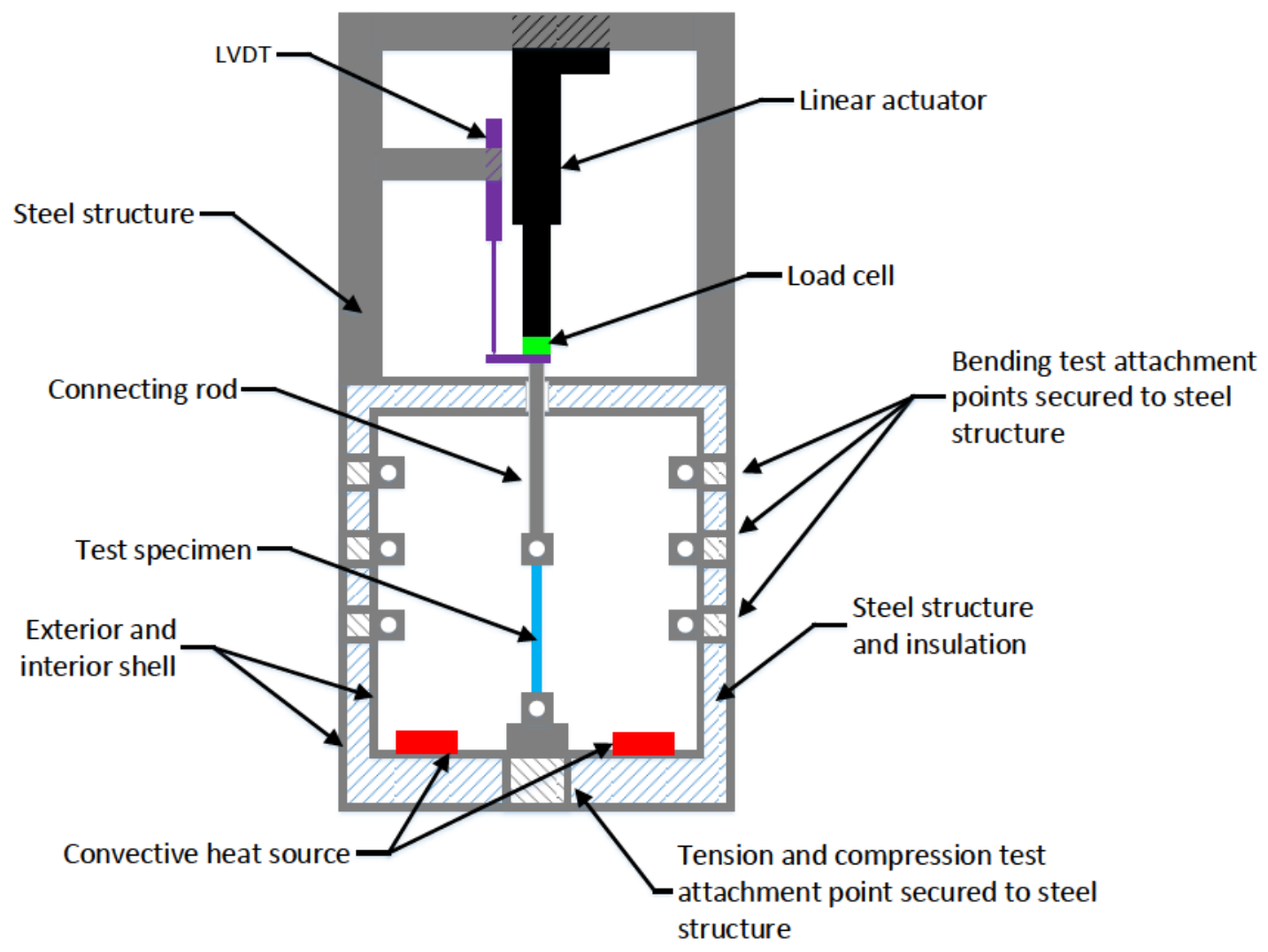

Figure 6.2: Conceptual drawing of the redesigned test setup

With the modifications made to the test equipment, further tests can be performed to fully characterize the response of the material.

\subsection{Future Work}

Without the restrictions bound by the original setup, tests can be performed at higher load, smaller temperature intervals, using fibre reinforcement as well as allow the tests to creep until failure occurs. To complete the characterization of the epoxy matrix, recovery data should be collected as the current life prediction assumes only the steady state stiffness of the material. To fully capture the resin's response to dynamic inputs, creep and relaxation tests should be performed at various loads, displacements and strain rates. Statistical analysis should also be performed as the small sample set limits the accuracy of the current characterization. In addition, characteristics such 
as the time dependency of the Poisson's ratio and creep failure should be collected.

Once the epoxy matrix has been fully characterized, bending tests should be conducted using scaled models of the composite wing spar to observe the effect a fully relaxed matrix has on the spar's ability to maintain its shape. This includes phenomena such as delaminations, debonding or inter ply buckling. Dynamic testing can also be performed on scaled models which can simulate actual flight conditions and to observe the viscoelastic memory effect in the matrix. 


\section{Chapter 7}

\section{Conclusion}

The research outlined in this thesis has provided a method to determine the long term response of composite materials. This concluding chapter will outline the key points and results of each section.

\subsection{Viscoelastic Response of MGS Epoxy System}

This thesis has shown that the response of MGS L285/H287 epoxy system can be characterized using the time temperature superposition principal and that a set of master curves can be generated for various service temperatures. The initial stiffness was determined to be $1100 \mathrm{MPa}$ which is one third the $3300 \mathrm{MPa}$ stated by the manufacturer. This discrepancy was attributed to the use of expired H287 hardener as well as the inability to observe the short term response of the material.

Using nonlinear regression techniques on a limited data set, only a one term Prony series was able to converge for both the creep and relaxation master curves. When comparing the creep and relaxation functions, the initial and final modulus were similar; however both underestimated the initial modulus by up to $41 \%$ when compared to the experimentally determined value. This variation is attributed to the lack of data points under 100 seconds when all the data has been shifted to its appropriate location. When comparing the predicted time constants, it is apparent that the compliance function produced a sensible fit; however the time constant determined for the relaxation function does not follow the experimental data. The relaxed moduli of the epoxy was determined to be approximately $10 \mathrm{MPa}$ and was calculated by averaging steady state values predicted by the compliance and relaxation functions. This steady state value was then used for analysis of the composite wing spar. 


\subsection{Response of Composite Wing Spar}

Structural analysis of the composite wing spar was performed using the classical lamination theory with modification to account for complex beam geometries. Due to proprietary reasons, the exact stacking sequence used to construct the spar was not available and basic assumptions were made based on expected flight loads. To validate these assumptions and the MATLAB algorithm created to analyse complex geometries, elastic properties were used and the response of the wing spar was compared to an expected range. Using the elastic properties, the angle of twist and the vertical and horizontal deflections at the wing tip during steady level flight was determined to be $0.15^{\circ}, 5.6 \mathrm{~cm}$ and $5.5 \mathrm{~cm}$ from their original positions respectively. Strength ratios for each ply were determined using various failure theories and were all well above 1. Once the elastic response was confirmed an analysis was performed using the steady state viscoelastic modulus.

If the matrix is assumed to be fully relaxed, the difference in the effective axial and flexural stiffness of the I-beam are $9.2 \%$ and $5.8 \%$ from their initial conditions respectively. This should be expected as the epoxy matrix carries a much smaller portion of the load when compared to the fibres. The torsional rigidity of the spar was calculated to be $42 \%$ lower than the elastic case; however this is expected as it is more dependant on the shear properties of the matrix. The angle of twist, vertical and horizontal wing tip deflections of the viscoelastic spar were determined to be $0.21^{\circ}, 5.9 \mathrm{~cm}$ and $5.8 \mathrm{~cm}$ respectively. When examining the Tsai-Hill failure theory, the strength ratio increased as the matrix relaxed as the limiting shear stress was redirected to the fibre-wise direction. If it is assumed that the ultimate strength of the epoxy decreases with time it would require a $99.9 \%$ decrease in strength for failure of the spar to occur. Strength ratios were determined through the maximum strain criteria and produced strength ratios similar to that of the elastic case. Stability analysis was performed and confirmed the compressive flange would resist buckling with a safety factor of 10.5 even though a $42 \%$ decrease in the critical buckling moment was observed. Currently the assumptions used in the stability analysis are in question and should be further examined. 


\subsection{Improvements, Future Work and Concluding Remarks}

To further validate the result of this thesis, the heating compartment of the testing apparatus should be reconstructed using the recommendation in Chapter 6 to improve the accuracy and diversity of the data set. Once the heating compartment has been constructed, bending tests should be conducted on a scaled wing spar to observe its actual response. This is especially important for the compressive flange as delaminations, debondings or inter-ply buckling of the fibres may occur. In addition, recovery data and the ultimate strength of the epoxy should be collected as limitation in the current set-up have prevented this.

Based on the assumptions and information presented in this thesis, Diamond's claims of unlimited service life for the DA-20 Kanata is possible assuming only the viscoelastic properties of the epoxy matrix. In the future, service life estimation should focus on typical failure modes such as from the accumulation of damage to the matrix and fibres or through delaminating or debonding effects. 


\section{List of References}

[1] R. Guedes, Creep and fatigue in polymer matrix composites. Woodhead Publishing Series in Composites Science and Engineering, Toronto, ON: Woodhead Publishing, March 2011.

[2] M. Goossens, Diamond DA20 Service Manual. Diamond Aircraft, London, ON, 2000.

[3] Cirrus, "Useful life and inspections." http://whycirrus.com/engineering/usefullife-inspections.aspx, December 2013.

[4] "Airworthiness manual chapter 523 - normal, utility, aerobatic and commuter category aeroplanes," tech. rep., Transport Canada, Ottawa, Canada.

[5] D. W. K. A.A.Baker, Composite Materials for Aircraft Structures. Reston, Virginia, USA: American Institute of Aeronautics and Astronautics, 2 ed., 2004.

[6] P. S. J. Sudah, S. Kumar and K. Vijayaraju, "Fatigue behavior of carbon fibre reinforced plastic under spectrum loading," Material Science and Engineering A, vol. 501, pp. 44-51, 2009.

[7] J. Laliberte, "Aero 4608: Composite materials." Course Notes, Carleton University, September 2013.

[8] U. S. G. A. Office, "Status of faas actions to oversee the safety of composite airplanes, gao-11-849," September 2011. http://www.hexcel.com/Resources/ContCarbon-Fiber-Data-Sheets.

[9] R. Talreja, "Studies on the failure analysis of composite materials with manufacturing defects," Mechanics of Composite Materials, vol. 49, no. 1, pp. 35-44, 2013.

[10] S. K. Mazumdar, Composites Manufacturing - Materials, Products and Process Engineering. New York, NY, USA: CRC Press LLC, 2002.

[11] M. C.-Y. Nui, Composite Airframe Structures. Adaso Adastra Engineering Center, 1992.

[12] S. Pant, Lamb Wave Propagation and Material Characterization of Metallic and Composite Aerospace Structures for Improved Structural Health Monitoring (SHM). PhD thesis. 
[13] K. K. Chawla, Composite Materials - Science and Engieering. New York, USA: Springer, 3 ed., 2012.

[14] P. Benham, R. Crawford, and C. Armstrong, Mechanics of Engineering Materials. Harlow, England: Prentice Hall, 2 ed., 1996.

[15] M. A. I. T.S. Srivatsan and R. Srinivasan, Fatigue of Materials II: Advances and Emergences in Understanding. Hoboken, New Jersey, USA: John Wiley Son, 1 ed., 2013.

[16] V. Kliman, "Fatigue life estimation under random loading using the energy criterion," International Journal of Fatigue, vol. 7, no. 1, pp. 39-44, 1985.

[17] "Sp-602 -environmental effects on advanced composite materials," tech. rep., American Socierty for Testing and Materials.

[18] L. B. H.F Brinson, Polymer Engineering Science and Viscoelasticity. Springer Science and Business Media, New York, NY, USA: Springer, 2008.

[19] S. K. Craig G. Merrett, Harry H. Hilton and E. S. Schwartz, "Suppression of bending-torsion flutter in accelerated flight with aero-servo-viscoelastic controls," Proceedings AIAA Atmospheric Flight Mechanics Conference,, 2011.

[20] C. G. Merrett and H. H. Hilton, "Influences of starting transients, aerodynamic definitions and boundary conditions on elastic and viscoelastic wing and panel flutter," Mathematics in Engineering, Science and Aerospace, vol. 2, no. 1, pp. 124, 2011.

[21] C. G. Merrett and H. H. Hilton, "Flutter initiation under steady-state and accelerated free stream velocities," SAE International Journal of Aerospace, vol. 4, no. 2, pp. 1449-1464, 2011.

[22] R. S. Lakes, Viscoelastic Solids. USA: CRC Press LLC, 1998.

[23] Hardies, "Introduction to polymers - classifications."

[24] D. Roylance, "Engineering viscoelasticity," October 2001. http://web.mit.edu/course/3/3.11/www/modules/visco.pdf.

[25] T. Chen, "Determining a prony series for a viscoelastic material from time varyinh strain data," Technical Memo 2000-210123, NASA, Hampton, VA, May 2000.

[26] J. Dealy and D. Plazek, "Time-temperature superposition - a user guide," Rheology Bulletin, vol. 78, no. 8, pp. 16-31, 2009.

[27] G. Odegard and A. Bandyopadhyay, "Physical aging of epoxy polymers and their composites," Journal of Polymer Science Part B: Polymer Physicse, vol. 49, no. 24, pp. 1695-1716, 2011.

[28] O. Learn", "Introduction to polymers - 5.3.1 time-temperature superposition," $2014 . \quad$ http://www.open.edu/openlearn/science-mathstechnology/science/chemistry/introduction-polymers/content-section-5.3.1. 
[29] H. H. Hilton, "The elusive and fickle viscoelastic poisson's ratio and its relation to the elastic-viscoelastic correspondence principle," Mathematical Science Publishers, vol. 4, no. 7-8, pp. 1314-1361, 2009.

[30] M. E. Tuttle, Structural Analysis of Polymeric Composite Materials. New York, USA: Marcel Bekker, Inc., 1 ed., 2004.

[31] L. C. Bank, Construction for Composites - Structural Design with FRP Materials. Hobokenk, NY, USA: John Wiley Sons, Inc., 2006.

[32] T. Purslow, "The shear properties of unidirectional carbon fibre reinforced plastics and their experimental determination," Technical Memo C.P. No. 1381, Procurement Executive, Ministry of Defence - Aeronautical Research Council, Farnborough, Hampshire, United Kingdom, 1977.

[33] L. P. Kollar, "Local buckling of fiber reinforced plastic composite structural members with open and closed cross sections," Journal of Structural Engineering, vol. 129, pp. 1503 - 1513, November 2003.

[34] W. W. J.L Chrsitian and B. Stein, "Standard test methods for tensile, compressive, and flexural creep and creep-rupture of plastics," tech. rep., American Socierty for Testing and Materials.

[35] C. McManis, "H-bridges: theory and practice," December 2006. http://www.mcmanis.com/chuck/robotics/tutorial/h-bridge/.

[36] D20.10, "D638-10 - standard test method for tensile properties of plastics," tech. rep., ASTM International.

[37] Hexcel, "Hewtow continuous carbon fibre," 2013. http://www.hexcel.com/Resources/Cont-Carbon-Fiber-Data-Sheets.

[38] M. Scheufler, "Specification for laminating resin 1285 with hardener 500." http://www.mgs-online.com/en/techinfo/ti $f$ rame.htm, December 2013.

[39] P.-D. Interglas, "Specification for e-glass type 92145," December 2013. http://www.pd-interglas.com/wordpress57sMHq7VK8/wpcontent/uploads/ITG-en $35 . p d f$. 